\title{
Dynamical vacuum energy in the expanding Universe confronted with observations: a dedicated study
}

\author{
Adrià Gómez-Valent, Joan Solà \\ High Energy Physics Group, Dept. ECM, and Institut de Ciències del Cosmos, \\ Univ. de Barcelona, Av. Diagonal 647, E-08028 Barcelona, Catalonia, Spain

\section{Spyros Basilakos} \\ Academy of Athens, Research Center for Astronomy and Applied Mathematics, \\ Soranou Efesiou 4, 11527, Athens, Greece \\ E-mails: adriagova@ecm.ub.edu, sola@ecm.ub.edu, svasil@academyofathens.gr
}

\begin{abstract}
Despite the many efforts, our theoretical understanding of the ultimate nature of the dark energy component of the universe still lags well behind the astounding experimental evidence achieved from the increasingly sophisticated observational tools at our disposal. While the canonical possibility is a strict cosmological constant, or rigid vacuum energy density $\rho_{\Lambda}=$ const., the exceeding simplicity of this possibility lies also at the root of its unconvincing theoretical status, as there is no explanation for the existence of such constant for the entire cosmic history. Herein we explore general models of the vacuum energy density slowly evolving with the Hubble function $H$ and/or its time derivative, $\rho_{\Lambda}=\rho_{\Lambda}(H, \dot{H})$. Some of these models are actually well-motivated from the theoretical point of view and may provide a rich phenomenology that could be explored in future observations, whereas some others have more limitations. In this work, we put them to the test and elucidate which ones are still compatible with the present observations and which ones are already ruled out. We consider their implications on structure formation, in combination with data on type Ia supernovae, the Cosmic Microwave Background, the Baryonic Acoustic Oscillations, and the predicted redshift distribution of cluster-size collapsed structures. The relation of these vacuum models on possible evidence of dynamical dark energy recently pointed out in the literature is also briefly addressed.
\end{abstract}

KEYWORDS: dynamical dark energy, cosmological constant, structure formation. 


\section{Contents}

1. Introduction 2

2. Time-evolving vacuum energy in an expanding universe 4

$\begin{array}{lll}\text { 3. Running vacuum versus time-evolving vacuum } & 7\end{array}$

4. Background solution of the cosmological equations 10

$\begin{array}{lll}4.1 & \text { Models A1 and A2 } & 10\end{array}$

4.2 Models B1 and B2 12

4.3 Models $\mathrm{C} 1$ and $\mathrm{C} 2 \quad 13$

$\begin{array}{lll}4.4 & \text { Including the effect of radiation } & 15\end{array}$

5. Linear perturbations for dynamical vacuum models $\quad 17$

5.1 Perturbation equations for dynamical vacuum models $\rho_{\Lambda}=\rho_{\Lambda}(t) \quad 18$

$\begin{array}{lll}5.2 & \text { Perturbations for type-A models } & 19\end{array}$

5.3 Perturbations for type-B and C models 20

6. Fitting the models to the observational data 21

6.1 The global fit to SNIa, CMB and BAOs 22

6.2 Adapting the BAO analysis for dynamical vacuum models 26

6.3 Combined likelihood function 27

6.4 Discussion of the fitting results and implications for dynamical DE 33

6.5 The linear growth rate of clustering and the $\gamma$ index 35

7. Testing the dynamics of vacuum through the cluster number counts $\begin{array}{ll}\text { method } & 38\end{array}$

7.1 Generalized Press-Schechter formalism 40

7.2 Numerical results: number counts of the dynamical vacuum models 45

$\begin{array}{lll}\text { 8. Discussion and conclusions } & 48\end{array}$

A. Understanding how the cluster number counts method works 51

B. Computing the collapse density threshold $\delta_{c}$ for the dynamical vacuum models 53

B.1 Type-A models $\quad 54$

B.2 Type-B models

B.3 Numerical procedure to determine $\delta_{c}$ 


\section{Introduction}

The existence of the dark energy (DE) component in our universe as the purported physical cause for the accelerated expansion is currently beyond dispute from the observational point of view [1]. The simplest explanation, namely in the form of a cosmological constant (CC) in Einstein's field equations, is apparently working quite well (it is the basis of the standard $\Lambda \mathrm{CDM}$ cosmological model), but unfortunately it does not provide a single clue about its origin in the context of fundamental physics, i.e. according to quantum field theory (QFT), string theory etc. In fact, the theoretical prediction within any sound framework is well-known to overshoot by far the observational value, and this dramatic discrepancy constitutes the famous CC problem [2] - see also the recent review [3].

Dynamical models of the DE offer better chances to mitigate the problem. Popular possibilities are, among others, quintessence and phantom energy in its various forms [4]. In the last few years many proposals for modified gravity theories have flourished with great impetus as an alternative to supersede the DE as a physical substratum, see e.g. [5] and references therein. In these models the DE appears usually as just a late-time effect in the history of the universe (and hence they overlook the impact of a huge vacuum energy density during the entire cosmic evolution). However, in subsequent modified gravity models it is possible to operate (at least technically) a dynamical adjustment of the DE value to its current size, irrespective of its initial value in the early universe, including a relaxation mechanism of the vacuum energy density in astrophysical domains such as the Solar System [6].

In another vein, there is the possibility that the DE is related to the vacuum energy density of, say, QFT in curved spacetime [7, 8]. In this case, the DE need not be just a constant in an expanding universe. In fact, one rather expects the vacuum energy density $\rho_{\Lambda}=\Lambda /\left(8 \pi G_{N}\right)$ to be a running quantity with the expansion rate. Such notion of dynamical vacuum energy can be formulated in more formal grounds inspired in the renormalization group $[9,10,11,12,13]$ (cf. $[3,14]$ for reviews), or from the more phenomenological point of view of time-evolving $\Lambda=\Lambda(t)$ or cosmological vacuum decay [15, 16, 17].

It is intriguing to think of the measured $\rho_{\Lambda}$ as tracing the energy density difference with respect to the flat spacetime vacuum, and if so $\Lambda$ should be of order of the present curvature, namely $R \sim H_{0}^{2}$. This interpretation has been emphasized in [3] where a parallelism is made with the Casimir effect. Recall that the net Casimir force is caused by the difference between the vibrational modes of the $\mathrm{QED}$ vacuum in between the plates as compared to its absence. Although the zero-point energy (ZPE) itself is not measurable (as it is infinite and this infinity is shared by the original vacuum state before we introduce the plates), "changes in the ZPE" are indeed detectable when we modify the boundary conditions. Similarly, we 
may view the evolution of the vacuum energy in an expanding background with (dynamical) curvature $R \sim H^{2}(t)$ as the change that remains of the disturbed vacuum energy density in the curved background after we remove the flat spacetime result - which is also contained in the curved spacetime calculation. This possibility could help to understand the CC problem from the renormalization framework in QFT [3].

Models of the aforementioned kind and related variants have been used to describe a smooth time-evolving vacuum energy density around its present value, $\rho_{\Lambda}^{0} \sim 10^{-47} \mathrm{GeV}^{4}$, and successfully compared with the modern cosmological observations on the background cosmology, including in some cases the effect of cosmic perturbations, see e.g. [18]-[23] and [24]-[28]. For recent additional studies of the ZPE in curved spacetime, cf. [29, 30]. Furthermore, a generalized class of the dynamical vacuum models has recently been applied to construct a complete history of the cosmological evolution starting from inflation up to the present days [31, 32].

Dynamical models of the vacuum energy may ultimately be necessary not only as a new paradigm to improve the theoretical status of the $\Lambda$-term in Einstein equations, but also phenomenologically so as to relax a number of tensions between the standard $\Lambda \mathrm{CDM}$ predictions and the observations that may be providing evidence of DE evolution (cf. the recent analysis of [33] based on the Baryonic Acoustic Oscillations as a tool to determine the expansion history of the universe). Last but not least, dynamical vacuum energy could also be linked to the frequent hints reported in the literature that the so-called fundamental constants of Nature might be slowly changing with the cosmic time [34, 35], see e.g. [36, 37] for a direct application of these ideas.

In this paper we focus on a large class of dynamical models of the vacuum energy inspired in QFT in curved spacetime, namely on those based on the structure $\rho_{\Lambda}(H, \dot{H})$ which is well-motivated for a Friedmann-Lemaitre-Robertson-Walker (FLRW)-like expanding universe characterized by the Hubble rate $H$. We solve not only their background cosmology but perform a detailed analysis of the corresponding cosmic perturbations and their implications for structure formation. For example, it is well-known that the so-called linear growth rate (the logarithmic derivative of the linear growth factor $D=\delta \rho_{m} / \rho_{m}$ with respect to $\ln a$, i.e. $f=d \ln D / d \ln a$ ), can be in some cases a good indicator of clustering, together with the growth rate index $\gamma$ (used as the effective parameterization of the growth rate through a power of the density parameter [38]). In general, $\gamma$ is a function of the scale factor or equivalently of the redshift, $z=(1-a) / a$, and the relation with the growth rate reads: $f(z)=\Omega_{m}(z)^{\gamma(z)}$. The asymptotic value of the growth rate index parameter takes distinctive values for different gravity models [39]. In the case of the concordance $\Lambda$ CDM model, $\gamma(0) \simeq 0.55-0.60$. In this work we examine these linear indicators of structure formation for the new models of the vacuum energy, but we find also very convenient to study nonlinear effects, such as the theoretically predicted cluster-size halo redshift distributions. These "number count" observables can help to break degeneracies between the 
vacuum models and can be especially useful in the context of realistic and future X-ray and Sunyaev-Zeldovich cluster surveys such as eROSITA [40] and SPT [41, 42], as shown in previous work [19, 20]. For other implications of dynamical models in the astrophysical domain, see e.g. [43].

We shall discuss the virtues and troubles associated to some of these vacuum models according to the Hubble terms involved in the dynamical structure of the vacuum energy. Let us emphasize that not all of the $\rho_{\Lambda}(H, \dot{H})$ models are equally favored from the theoretical point of view. Interestingly, those that are theoretically more favored are in fact the ones that best fit the structure formation data in combination with the other cosmic observables such as type Ia supernovae, the Cosmic Microwave Background and the Baryonic Acoustic Oscillations. At the same time we find models that perform comparatively not so good, and other that can be simply excluded by the current observations.

The plan of the paper is as follows. In Sec. 1 we define the theoretical framework for the dynamical vacuum models $\rho_{\Lambda}(H, \dot{H})$. In Sec. 2 we discuss the general dynamical vacuum models that depend on powers of the Hubble function and its time derivative. In Sect. 3 we single out the class of the running vacuum models, more closely related to QFT. The corresponding cosmological background solutions of these models is presented in Sect. 4. In the next section we formulate the linear cosmic perturbations for general dynamical vacuum models. The fitting of these models to the cosmic data is put forward in Sect. 6, where we also briefly address the implications that our dynamical vacuum models could have on possible evidence recently found on dynamical dark energy. In Sec. 7 we discuss how to distinguish the dynamical vacuum models by means of the redshift distribution of cluster-size halos. Finally, in Sect. 8 we provide our discussion and conclusions. In two appendices we furnish more technical details of our analysis, to wit: in Appendix A we discuss more closely why the cluster number counts method can crucially help to distinguish the dynamical vacuum models under consideration, and in Appendix B we summarize the calculation of the linear density threshold for collapse, $\delta_{c}$, for the models under consideration, a quantity that is crucially needed for the determination of the clustersize halo redshift distributions.

\section{Time-evolving vacuum energy in an expanding universe}

Let us consider the expanding universe as a perfect fluid with matter-radiation density $\rho_{m}$ and vacuum energy density $\rho_{\Lambda}$. The latter is usually associated to the value of the cosmological term through $\Lambda=8 \pi G \rho_{\Lambda}$, where $G$ is Newton's constant. While we assume that $G$ remains strictly constant here, we do not make the same assumption for $\rho_{\Lambda}$. The full energy-momentum tensor of the cosmic fluid can be written as $\tilde{T}_{\mu \nu} \equiv T_{\mu \nu}^{m}+T_{\mu \nu}^{\Lambda}$, where $T_{\mu \nu}^{m}$ is the ordinary matter energy-momentum tensor and $T_{\mu \nu}^{\Lambda}$ describes the vacuum part. The equation of state (EoS) for the matter component reads $p_{m}=\omega_{m} \rho_{m}$ (with $\omega_{m}=0$ 
for dust and $\omega_{m}=1 / 3$ for radiation), and we introduce also the corresponding EoS for the vacuum $p_{\Lambda}=\omega_{\Lambda} \rho_{\Lambda}$, which we discuss below.

In the flat FLRW metric, on which we will hereafter exclusively concentrate, the two independent gravitational field equations derived from Einstein's equations sourced by $\tilde{T}_{\mu \nu}$ are the following:

$$
3 H^{2}=8 \pi G\left(\rho_{m}+\rho_{\Lambda}\right)
$$

and

$$
2 \dot{H}+3 H^{2}=-8 \pi G\left(\omega_{m} \rho_{m}+\omega_{\Lambda} \rho_{\Lambda}\right),
$$

where the overdot denotes derivative with respect to cosmic time $t$. From these equations one can derive the rate of change of the Hubble function,

$$
\dot{H}=-4 \pi G\left[\rho_{m}\left(1+\omega_{m}\right)+\rho_{\Lambda}\left(1+\omega_{\Lambda}\right)\right] .
$$

A useful equation (actually a first integral) that follows from the original system (2.1)-(2.2) is the following:

$$
\dot{\rho}_{m}+\dot{\rho}_{\Lambda}+3 H\left(1+\omega_{m}\right) \rho_{m}+3 H\left(1+\omega_{\Lambda}\right) \rho_{\Lambda}=0 .
$$

All the above equations remain valid if we sum over all matter components (relativistic and nonrelativistic). In the frequent situation where there is a dominant matter component (e.g. cold matter or relativistic matter), it is possible to obtain the evolution law for the Hubble function in terms of the vacuum term and that matter component:

$$
\dot{H}+\frac{3}{2}\left(1+\omega_{m}\right) H^{2}=4 \pi G\left(\omega_{m}-\omega_{\Lambda}\right) \rho_{\Lambda}=\frac{1}{2}\left(\omega_{m}-\omega_{\Lambda}\right) \Lambda .
$$

By integrating this equation we can obtain $H$ in the relevant epoch of the cosmic evolution where that matter component dominates. This procedure will be frequently used in our analysis.

Equation (2.4) constitutes the local conservation of the full energy-momentum tensor $\tilde{T}_{\mu \nu}$ in the presence of all contributions of matter and vacuum energy, namely it expresses explicitly the covariant conservation law $\nabla^{\mu} \tilde{T}_{\mu \nu}=0$ in the FLRW metric. Such law holds for strictly constant $G$ since the left hand side of Einstein equations must have zero covariant derivative by virtue of the Bianchi identity. Being the result of an identity, Eq. (2.4) is not independent of the fundamental system of Friedmann-Lemaitre equations (2.1)-(2.2), but it is useful to provide a more physical interpretation of them.

Formally the above equations are valid whether the vacuum term $\rho_{\Lambda}$ is a rigid quantity or is represented by a dynamical variable $\rho_{\Lambda}=\rho_{\Lambda}(t)$. In the last case the energy density $\rho_{\Lambda}(t)$ could e.g. involve the dynamical behavior of various possible components of the dark energy and/or the effects of additional terms in the effective action, as for example in the modified gravity framework of Ref. [6] or in the $\Lambda$ XCDM model of [27], where in both cases the overall EoS parameter $\omega_{\Lambda}$ may have a nontrivial behavior. However, here we will assume that $\rho_{\Lambda}(t)$ is a true dynamical vacuum term whose time evolution is exclusively 
associated to the quantum effects on $\Lambda$ [9]. From this point of view, the corresponding EoS is still $\omega_{\Lambda}=-1$. This will be henceforth taken for granted.

In the present study the dynamical CC term is represented by a power series of the Hubble function and its time derivative:

$$
\Lambda(H)=c_{0}+\sum_{k=1} \alpha_{k} H^{k}+\sum_{k=1} \beta_{k} \dot{H}^{k}+\ldots
$$

where the leading term $c_{0}$ describes in good approximation the current universe and the other terms introduce a mild dynamical evolution. As we will see in the next section, the expression (2.6) generalizes previous forms that can be motivated within the class of running vacuum models [9]-[14], with certain restrictions, and hence they are placed in the general context of QFT in curved spacetime [7, 8, 44] - see [3] for a recent review focusing on these issues.

Lately these models have been successfully applied to describe the complete history of the universe, as they involve the ingredients capable of yielding a smooth transition from an early de Sitter stage to a proper radiation and matter epochs [31]. In practice, since structure formation is a relatively recent phenomenon, we limit ourselves to consider the lowest powers of $H$. Indeed, recall that the current vacuum energy density is of order $M_{P}^{2} H_{0}^{2}$, where $H_{0} \sim 10^{-42} \mathrm{GeV}$ is the current value of the Hubble parameter and $M_{P}=$ $1 / \sqrt{G} \sim 10^{19} \mathrm{GeV}$ is the Planck mass (in natural units). It follows that the power terms of (2.6) beyond $H^{2}$ and $\dot{H}$ are completely irrelevant for the present universe. Thus, terms of the form $H^{3}, \dot{H} H, H^{4}, \dot{H}^{2}, H^{2} \dot{H}, \ddot{H}$ etc. will be ignored for the present study, although they can be important for the early universe [31, 32, 45].

For the above models of dynamical vacuum energy the corresponding Eq. (2.4) simplifies as follows:

$$
\dot{\rho}_{m}+3\left(1+\omega_{m}\right) H \rho_{m}=-\dot{\rho_{\Lambda}} .
$$

Despite the obtained simplification, the nonvanishing r.h.s. of this equation signals a transfer of energy between vacuum and matter. Needless to say, this transfer is absent in the $\Lambda \mathrm{CDM}$ model for which $\rho_{\Lambda}=$ const. The nonvanishing time derivative of $\rho_{\Lambda}$ in the above conservation law involves the relevant powers of the Hubble function in the Eq. (2.6).

In this study we would like to check the effect of all terms that can be phenomenologically significant for the recent universe. Therefore, we will consider the linear term in $H$ as well as the $H^{2}$ and $\dot{H}$ terms. The linear terms, however, have a different status. They are not expected to have a fundamental origin within in QFT in curved space-time, a fact that actually applies to all the odd powers of the Hubble function [12, 13] as they are, in principle, incompatible with the general covariance of the effective action. However, the linear terms appear in various dark energy models in the presence of phenomenological bulk viscosity, see e.g. $[46,47,48]^{1}$.

\footnotetext{
${ }^{1}$ The linear terms also appear if nonperturbative infrared effects would be possible in the cosmological
} 
Therefore, following our aim to explore the various existing possibilities from the phenomenological point of view, we will test here the following list of six types or classes of dynamical vacuum models effectively leading to time-evolving $\Lambda$ scenarios:

$$
\begin{array}{ll}
A 1: & \Lambda=a_{0}+a_{2} H^{2} \\
A 2: & \Lambda=a_{0}+a_{1} \dot{H}+a_{2} H^{2} \\
B 1: & \Lambda=b_{0}+b_{1} H \\
B 2: & \Lambda=b_{0}+b_{1} H+b_{2} H^{2} \\
C 1: & \Lambda=c_{1} H+c_{2} H^{2} \\
C 2: & \Lambda=c_{1} \dot{H}+c_{2} H^{2}
\end{array}
$$

All of them can be, in principle, relevant for the study of the current and recent past cosmic history. Type A and B models, despite their differences, share one important feature, to wit: they all have a well-defined $\Lambda \mathrm{CDM}$ limit since they all tend to a constant value of $\Lambda$ when the coefficients of the powers of $H$ or $\dot{H}$ tend to zero. In contrast, models $\mathrm{C} 1$ and $\mathrm{C} 2$ can never behave as a rigid $\Lambda$ term. As we will see, this has important consequences for the phenomenological consistency of these models, when faced against the expansion and structure formation data.

\section{Running vacuum versus time-evolving vacuum}

In the current study we focus mainly on the class of a running vacuum energy models. By this denomination we mean not only that $\rho_{\Lambda}$ is a time-evolving quantity, but more specifically we assume that its evolution is inherited indirectly from another dynamical variable $\mu=\mu(t)$ on which $\rho_{\Lambda}$ is tied to, i.e. $\rho_{\Lambda}=\rho_{\Lambda}(\mu)$, rather than from a direct phenomenological law of the type $\rho_{\Lambda}=\rho_{\Lambda}(t)$. This point of view reminds of the renormalization group $(\mathrm{RG})$ running of the effective charges in gauge theories, and has been put forward in the context of cosmology in the literature $[9,10,11,12,13]$. In the cosmological context $\mu$ is a characteristic infrared cutoff scale typically associated to the Hubble rate, $H(t)$, as this quantity is of the order of the energy scale associated to the FLRW metric. In general $\mu^{2}$ is in correspondence with $H^{2}$ and also with $\dot{H} \equiv d H / d t$ (which has the same dimension as $H^{2}$ ). As shown in the previous references, one expects a RG equation for the vacuum energy density of the general form

$$
\frac{d \rho_{\Lambda}(\mu)}{d \ln \mu}=\frac{1}{(4 \pi)^{2}}\left[\sum_{i} B_{i} M_{i}^{2} \mu^{2}+\sum_{i} C_{i} \mu^{4}+\sum_{i} \frac{D_{i}}{M_{i}^{2}} \mu^{6}+\ldots\right],
$$

context, in a manner similar to QCD where lower dimensional terms squared in the gauge field, $A^{2}$, can appear together with the usual $F^{2} \sim\left(\partial A+A^{2}\right)^{2}$ ones, owing to the effect of IR renormalons. This issue is far from being established at present, but it has been considered in different ways in the literature, see e.g. $[49,50,51,52]$. 
where $M_{i}$ are the masses of the particles contributing in the loops, and $B_{i}, C_{i}, .$. are dimensionless parameters. The equation (3.1) gives the rate of change of the quantum effects on the $\mathrm{CC}$ as a function of the scale $\mu$. In practice we may cut off the series at the quadratic contributions, i.e. only the "soft-decoupling" terms of the form $\sim M_{i}^{2} \mu^{2}$ will remain for the current universe. The $M_{i}^{4}$ ones would trigger a too fast running of the CC term. Such effects are actually forbidden by the RG condition that only the fields satisfying $\mu>M_{i}$ are to be included as active degrees of freedom. Now, since $\mu$ is associated to a scale of order $H$ it is clear that such condition cannot be satisfied by any known particle mass in the current or recent universe. On the other hand the quartic contributions and higher are, as indicated above, are suppressed at this epoch ${ }^{2}$.

Let us recall that because of the general covariance of the effective action one expects only powers of $H^{2}$ and $\dot{H}$, whereas the linear terms in $H$ (and in general any term with an odd number of derivatives of the scale factor) are not expected [9, 12, 13]. This explains the general structure of the above RG equation.

Integrating (3.1) and relating $\mu^{2}$ to a linear combination of $H^{2}$ and $\dot{H}$ as the characteristic physical scale for the running [21], we can express the leading terms of the result as follows:

$$
\rho_{\Lambda}(H, \dot{H})=n_{0}+n_{\mathrm{i}} \dot{H}+n_{2} H^{2}=\frac{3}{8 \pi G}\left(C_{0}+C_{\dot{H}} \dot{H}+C_{H} H^{2}\right),
$$

where the second expression is convenient for the use of the new dimensionless coefficients $C_{H}$ and $C_{\dot{H}}$ that control the dynamical character of the vacuum energy density.

If we have a look to the list of vacuum models under study, Eq. (2.8), we observe that model A2 comprises the framework (3.2) that we have previously motivated from the RG equation, and model A1 is a particular case of A2. On the other hand models B1 and B2 involve a linear term in $\mathrm{H}$ which, as previously indicated, is not expected on fundamental grounds but could appear as an effective contribution.

Let us first turn our attention to model A1 in the list. It is the simplest model containing the expected ingredients. It is convenient to normalize the additive term such that it coincides with the value of the current $\mathrm{CC}$ density for $H=H_{0}$, and in addition we introduce a (dimensionless) parameter $\nu$, which plays the role of coefficient of the $\beta$ function for the running of the vacuum energy [9]. In this way the CC density for model A1 can be cast as follows:

$$
\rho_{\Lambda}(H)=\rho_{\Lambda}^{0}+\frac{3 \nu}{8 \pi} M_{P}^{2}\left(H^{2}-H_{0}^{2}\right) .
$$

As desired, $\rho_{\Lambda}\left(H=H_{0}\right)=\rho_{\Lambda}^{0}$. For $\nu=0$ the vacuum energy remains strictly constant at all times, $\rho_{\Lambda}=\rho_{\Lambda}^{0}$, whereas for non-vanishing $\nu$ there is an obvious evolution of the

\footnotetext{
${ }^{2}$ The main contribution to the running of $\rho_{\Lambda}$ clearly comes from the heaviest fields in a typical GUT near the Planck scale, i.e. those with masses $M_{i} \sim M_{X} \lesssim M_{P}$. See [12] for a specific scenario of this sort connected to the effective action of QFT in curved spacetime, and [3, 14] for a review.
} 
vacuum energy that departs as $H^{2}$ from a strictly constant value. This is a mild evolution provided $\nu$ is small enough. Obviously this model is a particular case of (3.2) with $C_{H}=\nu$ and $C_{\dot{H}}=0$. Substituting (3.3) in the general acceleration law for a FLRW-like universe in the presence of a vacuum energy density $\rho_{\Lambda}$, we find

$$
\frac{\ddot{a}}{a}=-\frac{4 \pi G}{3}\left(\rho_{m}+3 p_{m}-2 \rho_{\Lambda}\right)=-\frac{4 \pi G}{3}\left(1+3 \omega_{m}\right) \rho_{m}+C_{0}+\nu H^{2},
$$

where in this case

$$
C_{0}=\frac{8 \pi G}{3} \rho_{\Lambda}^{0}-\nu H_{0}^{2} .
$$

Let us next consider model A2. It generalizes the previous one by introducing the $\dot{H}$ contribution. Recall that the homogeneous terms $H^{2}$ and $\dot{H}$ are in general independent variables. From the two Friedmann's equations (2.1) and (2.2) it is easy to show that

$$
\frac{H^{2}}{\dot{H}}=-\frac{2}{3} \frac{1+r}{1+\omega_{m}}
$$

where $r=\rho_{\Lambda} / \rho_{m}$ is the ratio between the vacuum and matter energy densities. Even for the $\Lambda \mathrm{CDM}$ model (where $\rho_{\Lambda}$ is strictly constant) $r$ is a dynamical variable. At present $r \sim \mathcal{O}(1)(r \sim 7 / 3)$, whereas in the past $r \rightarrow 0$. In the radiation epoch $H^{2}$ was just minus half the value of $\dot{H}$, whereas at present $H_{0}^{2}$ is roughly minus twice the value of $\dot{H}_{0}$.

The acceleration equation for the scale factor of the model class A2 reads

$$
\frac{\ddot{a}}{a}=-\frac{4 \pi G}{3}\left(1+3 \omega_{m}\right) \rho_{m}+C_{0}+\nu H^{2}+C_{\dot{H}} \dot{H} .
$$

We still denote $C_{H} \equiv \nu$ since this parameter is closely related to the simplest running vacuum model (3.3)-(3.4) first introduced in [9]. Equation (3.7) can be rewritten in terms of the deceleration parameter $q$ and the usual cosmological parameters $\Omega_{m}=\rho_{m} / \rho_{c}$ and $\Omega_{\Lambda}=\rho_{\Lambda} / \rho_{c}$, where $\rho_{c}$ stands for the critical density $\rho_{c}=3 H^{2} / 8 \pi G$. We find:

$$
q=-\frac{\ddot{a}}{a H^{2}}=-1-\frac{\dot{H}}{H^{2}}=\frac{1}{2}\left(1+3 \omega_{m}\right) \Omega_{m}-\Omega_{\Lambda} .
$$

In the current epoch (where radiation can be safely neglected) we obtain the following relation, $C_{\dot{H}} \dot{H}_{0}=-C_{\dot{H}}\left(q_{0}+1\right) H_{0}^{2}=-(3 / 2) C_{\dot{H}} \Omega_{m}^{0} H_{0}^{2}$, which is now helpful to determine $C_{0}$ in (3.7) after we impose the boundary condition $\rho_{\Lambda}\left(H_{0}\right)=\rho_{\Lambda}^{0}$ in (3.2):

$$
C_{0}=H_{0}^{2}\left(\Omega_{\Lambda}^{0}-\nu+\frac{3}{2} \Omega_{m}^{0} C_{\dot{H}}\right)
$$

This relation clearly generalizes Eq. (3.5) for nonvanishing $C_{\dot{H}}$.

While models A (whether version A1 or the extended A2) are directly related to the RG approach based on Eq. (3.1), models B and C are more phenomenological by the reasons explained before. We will solve the cosmological equations for all these models in the next sections. 
Before solving these models, let us stress that in the case of the running vacuum models deriving from the general RG equation (3.1), the solution compatible with the general covariance of the effective action is of the form

$$
\rho_{\Lambda}(t)=c_{0}+\sum_{k=1} \alpha_{k} H^{2 k}(t)+\sum_{k=1} \beta_{k} \dot{H}^{k}(t),
$$

with $c_{0} \neq 0$. This is the particular form that Eq. (2.6) takes for the running vacuum case [3]. That is to say, one obtains in general an "affine" function constructed out of powers of $H^{2}=(\dot{a} / a)^{2}$ and $\dot{H}=\ddot{a} / a-H^{2}$, hence with an even number of time derivatives of the scale factor $a$. The higher order powers once more are irrelevant for the current universe and for this reason the model types A,B,C singled out above have been cut off at the lowest significant powers. As previously emphasized, the higher powers of $H$ can play a very significant role in the early universe. This role has been studied in detail in Refs. $[31,32]$ where it is shown in particular that they can lead to an inflationary scenario with graceful exit of the vacuum phase (de Sitter regime) into the radiation phase. It means that in this kind of dynamical vacuum fraweworks one can formulate a unified model of the cosmological evolution covering both the early, the recent and the present universe. For the rest of the paper we focus on the last two stretches of the cosmic history.

\section{Background solution of the cosmological equations}

Whenever possible we will solve the background equations for the models (2.8) by providing the matter and vacuum energy densities, as well as the Hubble function, in terms of the scale factor $a$ or the cosmic time. In general the most useful form is in terms of the scale factor since this is the variable which can be more easily related with the cosmological redshift $z=(1-a) / a$. However this will not always be possible and in some cases the analytic solution can be given only in terms of the cosmic time. In this section we provide the analytical solution of the background cosmologies corresponding to these models, obtained by extending the analysis of Refs. [19]-[23] to which we refer the reader for further details. The perturbations equations will be analyzed in subsequent sections.

\subsection{Models A1 and A2}

We start from the local covariant conservation law (2.7) in the matter dominated epoch and insert Eq. (3.2) on its r.h.s. A straightforward calculation making use of (2.3) and its time derivative yields:

$$
\dot{\rho}_{m}+3 H \frac{1-C_{H}}{1-\frac{3}{2} C_{\dot{H}}} \rho_{m}=0 .
$$

Trading the cosmic time variable for the scale factor through $d / d t=a H d / d a$ we can solve for the energy densities as a function of the scale factor as follows:

$$
\rho_{m}(a)=\rho_{m}^{0} a^{-3 \xi}
$$


and

$$
\rho_{\Lambda}(a)=\rho_{\Lambda}^{0}+\rho_{m}^{0}\left(\xi^{-1}-1\right)\left(a^{-3 \xi}-1\right)
$$

with

$$
\xi=\frac{1-\nu}{1-\alpha},
$$

where $\nu=C_{H}$ as before, and we have introduced $\alpha=3 C_{\dot{H}} / 2$. Obviously, for $\alpha=0$ model A2 becomes model A1 (for which $\xi=1-\nu$ ).

The corresponding Hubble function can now be obtained from the above energy densities using (2.1), resulting in the following expression:

$$
H^{2}(a)=H_{0}^{2}\left[1+\frac{\Omega_{m}^{0}}{\xi}\left(a^{-3 \xi}-1\right)\right] .
$$

It satisfies the correct normalization $H^{2}(a=1)=H_{0}^{2}$.

Let us also compute the corresponding inflection point where there is a transition of the Hubble expansion from the decelerating to the accelerating regime. From the definition (3.8) of deceleration parameter it is easy to show that it can be rewritten as follows:

$$
q=-1-\frac{a}{2 H^{2}(a)} \frac{d H^{2}(a)}{d a} .
$$

From this expression we can comfortably compute the point where $q=0$ from Eq. (4.5). Let us deliver the final result for model A2 in terms of the redshift value at the transition point:

$$
z_{I}=\left[\frac{2\left(\xi-\Omega_{m}^{0}\right)}{(3 \xi-2) \Omega_{m}^{0}}\right]^{1 / 3 \xi}-1 .
$$

The result for model A1 is just obtained by setting $\alpha=0$ (hence $\xi=1-\nu$ ). The standard $\Lambda \mathrm{CDM}$ result $z_{I}^{\Lambda \mathrm{CDM}}$ is, as always, recovered for $\xi=1$ :

$$
z_{I}^{\Lambda \mathrm{CDM}}=\left[\frac{2 \Omega_{\Lambda}^{0}}{\Omega_{m}^{0}}\right]^{1 / 3}-1 .
$$

The numerical value is $z_{I}^{\Lambda \mathrm{CDM}} \simeq 0.726(0.645)$ for $\Omega_{m}^{0}=0.28(0.31)$. As we can see the result is quite sensitive to the precision of $\Omega_{m}^{0}$ from observation ${ }^{3}$. Computing the departure of the new transition point (4.7) from the standard result for small $\nu$ and $\alpha$, we obtain:

$$
z_{I}-z_{I}^{\Lambda \mathrm{CDM}}=(\nu-\alpha)\left[\frac{2 \Omega_{\Lambda}^{0}}{\Omega_{m}^{0}}\right]^{1 / 3}\left[1+\frac{1}{3}\left(\ln \frac{2 \Omega_{\Lambda}^{0}}{\Omega_{m}^{0}}-\frac{1}{\Omega_{\Lambda}^{0}}\right)\right] .
$$

The numerical difference will be small to the extend that $\nu$ and $\alpha$ are small. The corresponding fit to these parameters will be made in Sect.6.

\footnotetext{
${ }^{3} \Omega_{m}^{0} h^{2}=0.1426 \pm 0.0025$, with $h=0.673 \pm 0.012$ for the standard $\Lambda$ CDM model from Planck $+\mathrm{WP}[1]$.
} 
Although the above equations provide the exact background solution of type-A models for arbitrary values of $\xi$, and hence of the original parameters $\nu$ and $\alpha$, the natural range for these parameters is

$$
|\nu| \ll 1, \quad|\alpha| \ll 1 .
$$

In this range the overall parameter $\xi$ can be expressed, in linear approximation in terms of an effective $\nu_{\text {eff }}$ parameter:

$$
\xi=\frac{1-\nu}{1-\alpha} \simeq 1-(\nu-\alpha) \equiv 1-\nu_{\text {eff }}
$$

\subsection{Models B1 and B2}

The vacuum energy density for model B2 can be parameterized as follows:

$$
\rho_{\Lambda}(H)=\frac{3}{8 \pi G}\left(C_{0}+C_{1} H+C_{2} H^{2}\right)
$$

The solution of model B1 obviously ensues by simply setting $C_{2}=0$ in the background solution of model B2. However, the technical difficulty in solving these models resides already in the simplest "affine" model B1 since the linear term in $H$ is harder to handle than the quadratic one. On the other hand, phenomenologically the reason to single out the case B1 is because this model is able to reasonable fit the data provided we maintain the additive term $C_{0} \neq 0$. For $C_{0}=0$, in contrast, the pure lineal model $\rho_{\Lambda} \propto H$ is unable to accommodate the data on structure formation [19, 23]. This feature has an important impact on some theoretical and phenomenological proposals in the literature [49, 50, 51, 52].

In order to solve model (4.12) analytically we proceed as follows. First of all it is convenient to re-express $\rho_{\Lambda}(H)$ in terms of dimensionless coefficients. We set $C_{1} \equiv \epsilon H_{0}$ (where $\epsilon$ is dimensionless) and we continue identifying $C_{2}$ with $\nu$, that is $C_{2} \equiv \nu$ as the basic parameter of the simplest viable model (3.3) compatible with the RG formulation. With this notation the expression that relates $C_{0}$ with $\nu$ and $\epsilon$ is:

$$
C_{0}=\frac{8 \pi G}{3} \rho_{\Lambda}^{0}-H_{0}^{2}(\epsilon+\nu)=H_{0}^{2}\left(\Omega_{\Lambda}^{0}-\epsilon-\nu\right)
$$

If we substitute (4.12) in the basic differential equation (2.5) for $H(t)$ in the matter dominated epoch, we find

$$
\frac{2}{3} \dot{H}+\zeta H^{2}-\epsilon H_{0} H=C_{0}
$$

where we have defined $\zeta=1-\nu$, not to be confused with Eq. (4.4). Upon direct integration we determine the Hubble function for this model explicitly in terms of the cosmic time:

$$
H(t)=\frac{H_{0}}{2 \zeta}\left[\mathcal{F} \operatorname{coth}\left(\frac{3}{4} H_{0} \mathcal{F} t\right)+\epsilon\right]
$$

with

$$
\mathcal{F}\left(\Omega_{\Lambda}^{0}, \epsilon, \nu\right) \equiv \sqrt{\epsilon^{2}+4 \zeta\left(\Omega_{\Lambda}^{0}-\epsilon-\nu\right)} .
$$


Notice the presence of an additive constant term in (4.15) (proportional to $\epsilon$ ) apart from the hyperbolic one. This feature is precisely what makes the treatment of the type-B models for $\rho_{\Lambda}(H)$ (the models with the linear term in $H$ ) more complicated from the technical point of view.

The time evolution of the pressureless matter density can be obtained from (2.3) and the explicit form of the Hubble function (4.15), with the result:

$$
\rho_{m}(t)=-\frac{\dot{H}(t)}{4 \pi G}=\frac{3 H_{0}^{2}}{32 \pi G \zeta} \mathcal{F}^{2} \operatorname{csch}^{2}\left(\frac{3}{4} H_{0} \mathcal{F} t\right)
$$

Similarly, from the previous equation and with the help of (4.15) and (2.1), we infer the expression of the vacuum energy density:

$$
\rho_{\Lambda}(t)=\frac{3 H_{0}^{2}}{32 \pi G \zeta^{2}}\left[\mathcal{F}^{2}+\epsilon^{2}+2 \epsilon \mathcal{F} \operatorname{coth}\left(\frac{3}{4} H_{0} \mathcal{F} t\right)+\nu \mathcal{F}^{2} \operatorname{csch}^{2}\left(\frac{3}{4} H_{0} \mathcal{F} t\right)\right] .
$$

Let us note that in the far past $(t \rightarrow 0)$ we have $\rho_{\Lambda}(t) / \rho_{m}(t) \simeq \nu /(1-\nu)$ and therefore since $|\nu| \ll 1$ the vacuum energy is suppressed in this period, as expected. The same conclusion applies in the radiation period, if we would include the corresponding radiation term. On the other hand, from (4.17) we have $\rho_{m}(t) \rightarrow 0$ for $t \rightarrow \infty$, as expected.

For this model it is impossible to obtain analytically the matter and vacuum energy densities in terms of the scale factor, except for $C_{0}=0$. We can however obtain $a(t)$ by direct integration of the Hubble function (4.15):

$$
a(t)=\left(\frac{\left[(2 \zeta-\epsilon)^{2}-\mathcal{F}^{2}\right]^{1+\frac{\epsilon}{\mathcal{F}}}}{\mathcal{F}^{2}(\mathcal{F}+2 \zeta-\epsilon)^{\frac{2 \epsilon}{\mathcal{F}}}}\right)^{\frac{1}{3 \zeta}} e^{\frac{\epsilon H_{0}}{2 \zeta} t} \sinh ^{\frac{2}{3 \zeta}}\left(\frac{3}{4} H_{0} \mathcal{F} t\right),
$$

where the complicated normalization factor (referred to as $A$ later on) is fixed by using $H\left(t_{0}\right)=H_{0}$ and $a\left(t_{0}\right)=1$. We can check that for $\epsilon=\nu=0$ it reduces to the standard $\Lambda \mathrm{CDM}$ one.

We remark that the two basic parameters $(\epsilon, \nu)$, or equivalently $(\epsilon, \zeta)$, of type-B models are completely independent and cannot be mimicked by a single effective parameter in a given matter-dominated or radiation-dominated epoch. This is different from type-A models, which can effectively be described by the unique parameter $\xi$, defined in (4.4), in the matter-dominated epoch.

Finally, one can show that type-B models have also an inflection point very close to that of the standard model for small $|\epsilon|$ and $|\nu|$. In particular, for type-B2 with $\epsilon \ll \nu$ the transition point is essentially given by Eq.(4.7) with $\xi=1-\nu$.

\subsection{Models C1 and C2}

Type $\mathrm{C} 1$ and $\mathrm{C} 2$ models are actually quite different from the previous ones and at the same time different from each other. They have in common the fact that do not have a well defined $\Lambda$ CDM limit for any value of the parameters, and therefore can never behave 
sufficiently close to a pure $\Lambda$ model. This raises some doubts about their possible viability, but they have nevertheless been discussed in the literature for different theoretical and phenomenological reasons.

For example, recently they have been discussed from the point of view of their possible relation with the "entropic-force" scenario [53] and its implications for the dark energy [54, 55]. Sometimes type-C models are presented in some drastically simplified forms as e.g. when only one of the two terms is present (say, models of the form $\Lambda \propto H$ or $\Lambda \propto H^{2}$ ), see e.g. $[49,50,51,52]$ and [56]. Here we will summarize very shortly the situation for completeness and also to highlight the important differences of type $\mathrm{C}$ with respect to type A and type B models.

Let us briefly comment on the model subclass $\mathrm{C} 2$ first. This is the canonical version of the mentioned class of models that acquired some relevance recently in connection to the entropic-force scenario and its possible cosmological implications [54, 55]. Many authors have analyzed recently this scenario for dark energy and generalizations thereof, cf. e.g. [57, 58, 59, 60, 61]. We mention in particular the studies in Ref. [21, 23], where it is shown in detail that model $\mathrm{C} 2$ is excluded. Basically, the acceleration parameter of this model remains constant, i.e., it does not change with the expansion. Thus this kind of model cannot have an inflection point from deceleration to acceleration.

In particular, models of the form $\rho_{\Lambda} \propto H^{2}$ or $\rho_{\Lambda} \propto \dot{H}$ are definitely ruled out. Recently it has been shown that even if the constant acceleration regime of model $\mathrm{C} 2$ would just be a partial description of a more complete model of expansion where a transition point would exist, the corresponding linear growth of cosmic perturbations is also strongly disfavored by the current data [23]. This result puts this specific kind of entropic-force cosmologies against the wall.

Let us now move to model C1. Let us summarize briefly the situation - (details are given in [23]. It is a particular case of B2 in the limit $C_{0} \rightarrow 0$ of Eq. (4.12), where in this case $C_{0}$ is defined in Eq. (4.13). Thus for this model we have $\Omega_{\Lambda}^{0}=\epsilon+\nu$, which implies that $\epsilon$ and $\nu$ cannot be both arbitrarily small. The Hubble function can be expressed in this case in terms of the scale factor:

$$
E(a)=1+\frac{\Omega_{m}^{0}}{\zeta}\left(a^{-3 \zeta / 2}-1\right)
$$

where we have defined the normalized Hubble rate $E(a) \equiv H(a) / H_{0}$. The matter and vacuum energy densities read:

$$
\rho_{m}(a)=\rho_{c}^{0}\left[\zeta E^{2}(a)-\left(\zeta-\Omega_{m}^{0}\right) E(a)\right]
$$

and

$$
\rho_{\Lambda}(a)=\rho_{c}^{0}\left[(1-\zeta) E^{2}(a)+\left(\zeta-\Omega_{m}^{0}\right) E(a)\right] .
$$


In the past $\rho_{\Lambda} / \rho_{m} \propto(1-\zeta) / \zeta=\nu /(1-\nu)$, and as a result we must require $|\nu|$ to be sufficiently small to avoid domination of vacuum energy. Since $\nu$ and $\epsilon$ cannot be simultaneously small for $\mathrm{C} 1$, we must have $|\nu| \ll \epsilon$ for this specific model class.

In contraposition to model $\mathrm{C} 2$, model $\mathrm{C} 1$ has a well-defined inflection point in the expansion regime. It is located at redshift

$$
z_{I}^{(\mathrm{C} 1)}=\left[\frac{2\left(\zeta-\Omega_{m}^{0}\right)}{(3 \zeta-2) \Omega_{m}^{0}}\right]^{2 / 3 \zeta}-1 .
$$

Because of $|\nu| \ll \epsilon$, all C1 models have an inflection point near the case $\nu=0$. For this situation we find e.g. $z_{I} \simeq 1.979(1.706)$ for $\Omega_{m}^{0}=0.28(0.31)$. These predictions are substantially different from the $\Lambda \mathrm{CDM}$ ones, see Eq. (4.8). From (4.21) and (4.20) we see that, in the past, the behavior of the matter density for the C1 models is abnormal: $\rho_{m}(a) \sim$ $\rho_{c}^{0} \zeta E^{2}(a) \sim \rho_{c}^{0}\left(\Omega_{m}^{0}\right)^{2} \zeta^{-1} a^{-3 \zeta}$. Even for $\nu=0$ (equivalently, $\zeta=1$, corresponding to the pure linear model $\left.\rho_{\Lambda} \propto H\right)$ there is an extra factor of $\Omega_{m}^{0}$ as compared to the $\Lambda$ CDM. Because of this anomaly, when the model is confronted with the cosmological data the preferred value of $\Omega_{m}^{0}$ is significantly larger than in the $\Lambda \mathrm{CDM}$, see [23] and [19].

Needless to say, for a full assessment of the possibilities of the various models we have to consider the analysis of cosmic perturbations. We do this in Sect. 5 .

\subsection{Including the effect of radiation}

Up to now we have not considered the effect of relativistic matter in the solution of the background cosmological equations of the dynamical vacuum models since we have focused on the form of the solution near our time. For the study of cosmological perturbations in Sect. 5 the effect of radiation is not necessary. However, for the fitting of the current models to the Baryonic Acoustic Oscillations (BAO) and the Cosmic Microwave Background $(\mathrm{CMB})$ in Sect.6 it is convenient to test this effect.

Including the radiation component in Eq. (2.4) leads to

$$
\dot{\rho}_{m}+\dot{\rho}_{r}+\dot{\rho}_{\Lambda}+3 H \rho_{m}+4 H \rho_{r}=0 .
$$

For type-A vacuum models, where $\rho_{\Lambda}$ is given by (3.2), we obtain the following generalized form of Eq. (4.1):

$$
\dot{\rho}_{m}+3 H \xi \rho_{m}+\dot{\rho}_{r}+4 H \xi^{\prime} \rho_{r}=0,
$$

where we used the familiar parameter $\xi$, defined in (4.4), and we have now introduced a new one that is related with the radiation component:

$$
\xi^{\prime}=\frac{1-\nu}{1-4 \alpha / 3}
$$

We do not want to describe in detail the exchange dynamics of matter and radiation here (despite it was certainly relevant in some epoch of the universe), but only the situation 
when one of the components dominates. We can therefore solve separately for the matter and radiation parts of (4.25) - the same kind of assumption as in the standard model case. In this way we obtain:

$$
\begin{aligned}
\rho_{m} & =\rho_{m}^{0} a^{-3 \xi} \\
\rho_{r} & =\rho_{r}^{0} a^{-4 \xi^{\prime}} .
\end{aligned}
$$

The presence of $\xi$ and $\xi^{\prime}$, when different from 1, denotes the anomaly in the corresponding conservation laws. Of course such anomaly must be small (i.e. $\xi \simeq \xi^{\prime} \simeq 1$ ) in the natural physical region (4.10), but the small deviation from 1 is exactly what permits the vacuum energy to evolve with the expansion. By repeating the integration procedure, the vacuum energy density in the presence of radiation evolves as follows:

$$
\rho_{\Lambda}(a)=\rho_{\Lambda}^{0}+\rho_{m}^{0}\left(\xi^{-1}-1\right)\left(a^{-3 \xi}-1\right)+\rho_{r}^{0}\left(\xi^{\prime-1}-1\right)\left(a^{-4 \xi^{\prime}}-1\right) .
$$

Similarly, the corresponding normalized Hubble rate in the presence of radiation reads

$$
E^{2}(a)=1+\frac{\Omega_{m}^{0}}{\xi}\left(a^{-3 \xi}-1\right)+\frac{\Omega_{r}^{0}}{\xi^{\prime}}\left(a^{-4 \xi^{\prime}}-1\right)
$$

where the various normalized cosmological parameters satisfy now the constraint

$$
\Omega_{m}^{0}+\Omega_{r}^{0}+\Omega_{\Lambda}^{0}=1
$$

Obviously we have found a generalizion of the equations of Sect. 4.1 for when the radiation component is taken into account.

Consider now type-B models. As already mentioned, in this case we cannot avoid using the cosmic time variable for the analytical solution. Under similar assumptions as before, we obtain the corresponding matter densisties as follows:

$$
\begin{aligned}
& \rho_{m}(t, a)=\rho_{m}^{0} e^{\frac{3}{2} \epsilon H_{0}\left(t-t_{0}\right)} a^{-3 \zeta} \\
& \rho_{r}(t, a)=\rho_{r}^{0} e^{2 \epsilon H_{0}\left(t-t_{0}\right)} a^{-4 \zeta},
\end{aligned}
$$

where $\zeta=1-\nu$ and $t_{0}$ is the value of the cosmic time at present.

We assume that the effects of radiation are relatively small in the relevant periods of our interest and hence cannot modify dramatically the matter-dominated solution. Such is the case around the time when the CMB was released. To generate a consistent solution within this period, we initially assume that the scale factor evolves with time as in the nonrelativistic epoch and solve for it. Introducing the result in (4.32) and (4.33) we obtain:

$$
\begin{gathered}
\rho_{m}(t)=\rho_{m}^{0} B e^{-\frac{3}{2} \epsilon H_{0} t_{0}} \operatorname{csch}^{2}\left(\frac{3}{4} H_{0} \mathcal{F} t\right), \\
\rho_{r}(t)=\rho_{r}^{0} B^{4 / 3} e^{-2 \epsilon H_{0} t_{0}} \operatorname{csch}^{8 / 3}\left(\frac{3}{4} H_{0} \mathcal{F} t\right),
\end{gathered}
$$


where the normalization constant is $B=A^{-3 \zeta}$, with $A$ the one in Eq. (4.19).

The Hubble function including the effects of radiation in the matter-dominated epoch can now be computed from

$$
H^{2}(t)=\frac{8 \pi G}{3}\left[\rho_{m}(t)+\rho_{r}(t)+\rho_{\Lambda}(t)\right]=\frac{8 \pi G}{3}\left[\rho_{m}(t)+\rho_{r}(t)\right]+C_{0}+C_{1} H(t)+C_{2} H^{2}(t),
$$

where we have used (4.12) and we understand that $\rho_{m}(t)$ and $\rho_{r}(t)$ are given by (4.34) and (4.35) respectively. After some calculations we arrive at the following result:

$$
H(t)=\frac{H_{0}}{2 \zeta}\left[\epsilon+\sqrt{\epsilon^{2}+4 \zeta s(t)}\right]=\frac{H_{0}}{2 \zeta}\left[\epsilon+\mathcal{F} \operatorname{coth}\left(\frac{3}{4} H_{0} \mathcal{F} t\right) \sqrt{1+\Delta(t)}\right],
$$

where

$$
\Delta(t)=\frac{\Omega_{r}^{0}}{\Omega_{m}^{0}}\left(\frac{\mathcal{F}^{2}}{4 \zeta \Omega_{m}^{0}}\right)^{1 / 3} \operatorname{csch}^{2 / 3}\left(\frac{3}{4} H_{0} \mathcal{F} t\right) \operatorname{sch}^{2}\left(\frac{3}{4} H_{0} \mathcal{F} t\right) .
$$

The form of the result (4.37) enables us to better compare with the original form (4.15). The correction from the radiation is clearly represented by the function $\Delta(t)$. Obviously this effect is negligible at the present time since the prefactor in it reads $\Omega_{r}^{0} / \Omega_{m}^{0}=$ $\left(1+0.227 N_{\nu}\right) \Omega_{\gamma}^{0} / \Omega_{m}^{0}=4.15 \times 10^{-5} h^{-2} / \Omega_{m}^{0} \simeq 3 \times 10^{-4}$ (including photons and $N_{\nu}=3$ neutrino species, and assuming $\Omega_{m}^{0} h^{2} \simeq 0.14$ ). However, at decoupling (i.e. at the time $t_{*}$ of last scattering of radiation with matter) the hyperbolic function in (4.38) rockets into a numerical value of order $\sim 10^{3}$ and $\Delta\left(t_{*}\right)$ can become quite sizeable. In fact, the fraction of radiation at decoupling can be around $\sim 23 \%$ in the $\Lambda$ CDM case. This is of course also the case for the generalized type $\mathrm{A}$ and $\mathrm{B}$ vacuum models deviating mildly with respect to the $\Lambda$ CDM (i.e. for small values of the parameters).

From the numerical integration of (4.37) we can obtain the corresponding improved version of the scale factor, and with it the function $H(a)$ and the energy densities $\rho_{m}(a)$ and $\rho_{\Lambda}(a)$ numerically.

\section{Linear perturbations for dynamical vacuum models}

After discussing the most relevant aspects of the background cosmological solution, the next essential step in our study is to analyze the linear perturbations equations. The structure formation properties obviously play an essential role to discriminate between the three kinds $\mathrm{A}, \mathrm{B}$ and $\mathrm{C}$ of dynamical vacuum models considered in this paper. In this section we discuss the perturbations in the presence of a variable vacuum energy. While we are not going to introduce perturbations for the vacuum energy itself, only for matter, we incorporate the dynamical character of $\rho_{\Lambda}$ in the matter perturbation equations. In other words, $\rho_{\Lambda}=\rho_{\Lambda}(t)$ is time evolving, but homogeneous in first approximation. This approach will suffice to clarify the fingerprint differences between the A,B and $\mathrm{C}$ model types as far as structure formation is concerned. 


\subsection{Perturbation equations for dynamical vacuum models $\rho_{\Lambda}=\rho_{\Lambda}(t)$}

We consider the linear perturbation equations for a system composed of the dynamical vacuum fluid $\rho_{\Lambda}=\rho_{\Lambda}(t)$ and the matter fluid $\rho_{m}=\rho_{m}(t)$, assuming that there are matter perturbations $\delta \rho_{m}$ but no perturbation in $\rho_{\Lambda}$. Although the inclusion of perturbations of the $\rho_{\Lambda}$ component is possible $[24,25,26]$, it is in general model-dependent and is not necessary for the present study. In the synchronous gauge the relevant system of first order differential equations in the epoch of structure formation can be extracted from the general framework of [25] as follows ${ }^{4}$ :

$$
\begin{aligned}
& \ddot{h}+2 H \dot{h}=-8 \pi G \delta \rho_{m} \\
& \delta \dot{\rho}_{m}+\rho_{m}\left(\theta_{\mathrm{m}}+\frac{\dot{h}}{2}\right)+3 H \delta \rho_{m}=0 \\
& \rho_{m} \dot{\theta}_{m}+\left(\dot{\rho}_{m}+5 H \rho_{m}\right) \theta_{\mathrm{m}}=0,
\end{aligned}
$$

where $h$ is the trace of the metric perturbation $\delta g_{\mu \nu}$, and $\theta_{\mathrm{m}}=\nabla_{\mu} \delta U^{\mu}$ is the divergence of the perturbed matter velocity. The last equation of the system (5.1) can be cast as

$$
\dot{\theta}_{m}+(2 H+\Psi) \theta_{\mathrm{m}}=0
$$

where we have used Eq. (2.7), and defined

$$
\Psi \equiv-\frac{\dot{\rho}_{\Lambda}}{\rho_{m}}=3 H+\frac{\dot{\rho}_{m}}{\rho_{m}}
$$

Introducing the linear growth factor $D \equiv \delta \rho_{m} / \rho_{m}$, the first two equations of the system (5.1) can be combined with (5.2) to yield the desired second order differential equation for $D$ in the presence of a dynamical vacuum term:

$$
\ddot{D}+(2 H+\Psi) \dot{D}-\left(4 \pi G \rho_{m}-2 H \Psi-\dot{\Psi}\right) D=\Psi \theta_{\mathrm{m}},
$$

In the $\Lambda$ CDM model we have $\rho_{\Lambda}=$ const. and hence $\Psi=0$, so that the above equation correctly shrinks to the standard one [38]:

$$
\ddot{D}+2 H \dot{D}-4 \pi G \rho_{m} D=0 \text {. }
$$

We will henceforth set the r.h.s. of (5.4) to zero. For the vacuum models under consideration the parameters $p_{i}=\nu, \alpha, \epsilon$ of the various models under consideration are small, $\left|p_{i}\right| \ll 1$. Taking into account that $\theta_{m}$ is a perturbation term and that $\dot{\rho}_{\Lambda}$ is proportional to at least one $p_{i}$ (as otherwise $\rho_{\Lambda}$ would remain constant and $\Psi=0$ ), the product $\left|\Psi \theta_{\mathrm{m}}\right| \sim\left|\mathcal{O}\left(p_{i}\right) \theta_{\mathrm{m}}\right|$ is of second order and can be neglected.

\footnotetext{
${ }^{4}$ In previous studies [24] it was found that the perturbation equations in two different gauges (synchronous and conformal Newtonian) leads to perfectly consistent results for this kind of models.
} 
In this approximation, we can rewrite the homogeneous form of (5.4) in terms of the scale factor as independent variable as follows:

$$
D^{\prime \prime}(a)+\left[\frac{3}{a}+\frac{H^{\prime}(a)}{H(a)}+\frac{\Psi(a)}{a H(a)}\right] D^{\prime}(a)-\left[\frac{4 \pi G \rho_{m}(a)}{H^{2}(a)}-\frac{2 \Psi(a)}{H(a)}-a \frac{\Psi^{\prime}(a)}{H(a)}\right] \frac{D(a)}{a^{2}}=0,
$$

where primes here indicate $d / d a$ differentiation, and we have traded the cosmic time variable for the scale factor through $d / d t=a H(a) d / d a$. In particular, notice that when $p_{i}=0$ we have $\Psi=0$ and (5.6) reduces to the standard perturbation equation for the $\Lambda \mathrm{CDM}$ [62]:

$$
D^{\prime \prime}(a)+\left[\frac{3}{a}+\frac{H^{\prime}(a)}{H(a)}\right] D^{\prime}(a)-\frac{3}{2} \Omega_{m}^{0} \frac{H_{0}^{2}}{H^{2}(a)} \frac{D(a)}{a^{5}}=0,
$$

where in the last term of the l.h.s. we have used the fact that matter is covariantly conserved in the $\Lambda \mathrm{CDM}$ and hence $\rho_{m}=\rho_{m}^{0} a^{-3}$. The decaying mode solution can be shown to be $D \propto H$, but this is not the one we want. The growing mode solution of (5.7), which is the relevant one, is well-known and reads as follows:

$$
D(a)=\frac{5}{2} \Omega_{m}^{0} E(a) \int_{0}^{a} \frac{d a^{\prime}}{\left(a^{\prime} E\left(a^{\prime}\right)\right)^{3}},
$$

where as before $E(a) \equiv H(a) / H_{0}$. Early on in the matter dominated epoch, when $E(a)=$ $\sqrt{\Omega_{m}^{0}} a^{-3 / 2}$, eq.(5.8) yields the standard result for the linear growth factor: $D(a) \propto a$. The effect of a nonvanishing $\rho_{\Lambda}>0$ is to suppress this linear growing rate, and in our case this effect is dynamical since $\rho_{\Lambda}=\rho_{\Lambda}(t)$.

\subsection{Perturbations for type-A models}

In the following we solve the perturbation equation (5.6) for model A2 and then derive the solution of A1 as a particular case. Recall that for these models the background solution can be fully expressed in terms of the combined parameter $\xi$, which depends on $\nu$ and $\alpha$ as indicated in (4.4). It is convenient to introduce the following change of independent variable, which can be operated on the cosmic time or the scale factor as follows:

$$
x=\operatorname{coth}\left[\frac{3}{2} H_{0} \sqrt{\xi\left(\xi-\Omega_{m}^{0}\right)} t\right]
$$

and

$$
x^{2}=\frac{\xi}{\xi-\Omega_{m}^{0}} E^{2}(a)=1+\frac{\Omega_{m}^{0}}{\xi-\Omega_{m}^{0}} a^{-3 \xi} .
$$

These changes of variable are associated respectively to the perturbation equations (5.4) and (5.6). Starting from any of these equations and applying the corresponding change of variable (5.9) or (5.10) we arrive, after some lengthy algebra, at the following result:

$$
\begin{aligned}
3 \xi^{2}\left(x^{2}-1\right)^{2} \frac{d^{2} D(x)}{d x^{2}} & +2 \xi(6 \xi-5) x\left(x^{2}-1\right) \frac{d D(x)}{d x} \\
& -2\left[(2-\xi)(3 \xi-2) x^{2}-\xi(4-3 \xi)\right] D(x)=0 .
\end{aligned}
$$


Notice that for $\alpha=0$ (hence $\xi=\zeta=1-\nu$ ) the previous equation reduces to the one for the type-A1 model, first analyzed in Ref. [19]. Thus, we have extended the perturbations analysis of that reference so as to include the more general class of models A2, which had not been considered before. We find that the basic parameter of models A1 and A2 are $\zeta$ and $\xi$ respectively, and this holds both at the background and perturbation levels. Remarkably, the basic perturbation equation turns out to be formally the same in each model after exchanging the parameters $\zeta(\mathrm{A} 1) \leftrightarrow \xi(\mathrm{A} 2)$. Not only so, this means that type A2 model effectively behaves as a single parameter model $\xi$ for all purposes.

The solution of 5.11 can be expressed as follows:

$$
D(x)=\left(x^{2}-1\right)^{\frac{5-3 \xi}{6 \xi}} Q_{n}^{m}(x),
$$

where $Q_{n}^{m}(x)$ is the associated Legendre's function of the second kind, and

$$
m=\frac{1}{3 \xi}-1 \quad n=\frac{1}{3 \xi} .
$$

Using standard properties of the Legendre functions [63] (see also Appendix B of Ref. [19]) and restoring the scale factor variable through Eq. (5.10), we can finally express the solution within the natural parameter domain (4.10) in the following way:

$$
D(a)=A_{1} a^{\frac{9 \xi-4}{2}} E(a) F\left(\frac{1}{3 \xi}+\frac{1}{2}, \frac{3}{2} ; \frac{1}{3 \xi}+\frac{3}{2} ;-\frac{\xi-\Omega_{m}^{0}}{\Omega_{m}^{0}} a^{3 \xi}\right),
$$

where $A_{1}$ is a constant to be adjusted by an initial condition, and $F$ is the conventional hypergeometric series [63].

Recall that the natural range of the parameters for type-A models is given by Eq. (4.10), where $\nu_{\text {eff }}$ is the single effective parameter. Thus, in practice we can replace $\xi \rightarrow \zeta_{\text {eff }}$ in the equations (5.11) and (5.14), where $\zeta_{\text {eff }} \equiv 1-\nu_{\text {eff }}$.

Let us note that, in the particular case $\nu=\alpha=0$ (hence $\zeta_{\text {eff }}=1$ ), the formula (5.14) leads to the $\Lambda \mathrm{CDM}$ solution (5.8) after using a standard integral representation of the hypergeometric series [63]. In the early epochs of matter domination, i.e. for sufficiently large values of $a$ when the cosmological term can be neglected and $E(a) \simeq \sqrt{\Omega_{m}^{0}} a^{-3 / 2}$, the solution (5.14) takes on the simple form $D(a) \sim a$. This behavior was also obtained previously from (5.8) and it can be used as initial condition to determine $A_{1}$.

Finally, let us mention that in Sect.7 (and Appendix B), we will extend the structure formation analysis to the non-linear regime (specifically to the formation of collapsed structures).

\subsection{Perturbations for type-B and C models}

The corresponding perturbations analysis starts in this case directly from Eq. (5.4). It proves convenient the following change of independent variable

$$
y(t)=\operatorname{coth}\left(\frac{3}{4} H_{0} \mathcal{F} t\right),
$$


The matter density (4.17) in terms of the new variable reads

$$
\rho_{m}(y)=\frac{3 H_{0}^{2}}{32 \pi G \zeta} \mathcal{F}^{2}\left(y^{2}-1\right) .
$$

Consider next the function $\Psi$ defined in (5.3). With the help of (4.18) and after straightforward algebra we can write it also in terms of $y$ and express the result in a rather compact form:

$$
\Psi(y)=-\frac{\dot{\rho}_{\Lambda}(t)}{\rho_{m}(t)}=\frac{3 H_{0}}{2 \zeta}\left[\epsilon+(1-\zeta) \mathcal{F} \operatorname{coth}\left(\frac{3}{4} H_{0} \mathcal{F} t\right)\right]=\frac{3 H_{0}}{2 \zeta}[y(1-\zeta) \mathcal{F}+\epsilon] .
$$

With the above formulae and performing the substitution (5.15) in the original Eq. (5.4) we arrive at the differential equation for the growth factor $D(y)$ in the new variable:

$$
\begin{aligned}
\left(y^{2}-1\right) \frac{d^{2} D(y)}{d y^{2}} & +\left[\frac{2 y}{3 \zeta}(6 \zeta-5)-\frac{10 \epsilon}{3 \zeta \mathcal{F}}\right] \frac{d D(y)}{d y} \\
& +\frac{2}{3 \zeta}\left[\frac{4}{\zeta\left(y^{2}-1\right)}\left(\frac{\epsilon^{2}}{\mathcal{F}^{2}}+(1-\zeta) y^{2}+\frac{\epsilon}{\mathcal{F}}(2-\zeta) y\right)+3 \zeta-4\right] D(y)=0
\end{aligned}
$$

This equation cannot be solved analytically, not even in terms of standard special functions. The case of the simpler type-B1 model, for which $\nu=0$, is no exception. Thus, for both types B1 and B2 we are forced to use the numerical techniques, for instance the standard method of finite differences.

During the matter-dominated epoch $D(a) \propto a$, so we take as initial conditions the value of the growth factor and its derivative at very high redshifts $y_{i}=y\left(z_{i} \gg z^{*}\right.$ ) (recall that at redshifts of order $z^{*}=\mathcal{O}(1)$ the vacuum energy density begins to dominate the Universe's dynamics for the $\Lambda \mathrm{CDM}$ and the other models considered here, see Sect. 4). The scale factor can be expressed in terms of $y$ with the help of (4.19):

$$
a(y)=A\left(y^{2}-1\right)^{-\frac{1}{3 \zeta}}\left(\frac{y+1}{y-1}\right)^{\frac{\epsilon}{3 \zeta \mathcal{F}}} .
$$

We normalize the growth factor with the value $D(z=0)$, i.e. $D(a=1)$, and we take $D(a)=a$ at very high redshifts.

Concerning type- $\mathrm{C} 1$ models the perturbation equations have been studied in detail in Ref. [23]. We have explicitly checked that the results of that reference are correctly retrieved from the general Eq. (5.18) in the limit $C_{0} \rightarrow 0$ in Eqs. (4.12) and (4.13). Finally, we recall that in Ref. [23] the perturbations analysis of type-C2 model was also considered and it was shown that it does not lead to a growing mode solution for any reasonable value of the cosmological parameters, which is a fatal blow for the C1 class.

\section{Fitting the models to the observational data}

In the following, we describe the statistical method, the observational samples and data statistical analysis that will be adopted to constrain the parameters of the dynamical vacuum models presented in the previous sections. We extract our fit from the combined data 
on type Ia supernovae (SNIa), the shift parameter of the Cosmic Microwave Background (CNB), and the data on the Baryonic Acoustic Oscillations (BAOs). The basic fitting results to the dynamical models under consideration are presented in a nutshell in Figs. 1-4 and Tables 1 and 2. We devote the rest of this section to explain these results and also to analyze the implications for linear structure formation.

\subsection{The global fit to SNIa, CMB and BAOs}

First of all, we use the Union 2.1 set of 580 type Ia supernovae of Suzuki et al. [64]. The corresponding $\chi_{\mathrm{SNIa}}^{2}$ function, to be minimized, is:

$$
\chi_{\mathrm{SNIa}}^{2}(\mathbf{p})=\sum_{i=1}^{580}\left[\frac{\mu_{\mathrm{th}}\left(z_{i}, \mathbf{p}\right)-\mu_{\mathrm{obs}}\left(z_{i}\right)}{\sigma_{i}}\right]^{2},
$$

where $z_{i}$ is the observed redshift for each data point. The fitted quantity $\mu$ is the distance modulus, defined as $\mu=m-M=5 \log d_{L}+25$, in which $d_{L}(z, \mathbf{p})$ is the luminosity distance:

$$
d_{L}(z, \mathbf{p})=c(1+z) \int_{0}^{z} \frac{\mathrm{d} z^{\prime}}{H\left(z^{\prime}\right)},
$$

with $c$ the speed of light (now included explicitly in some of these formula for better clarity) and $\mathbf{p}$ a vector containing the cosmological parameters of the models that we wish to fit for. In equation (6.1), the theoretically calculated distance modulus $\mu_{\text {th }}$ for each point follows from using (6.2), in which the Hubble function is the one corresponding to each model, see Sect. 4 . Finally, $\mu_{\mathrm{obs}}\left(z_{i}\right)$ and $\sigma_{i}$ stand for the measured distance modulus and the corresponding $1 \sigma$ uncertainty for each SNIa data point, respectively. The previous formula (6.2) for the luminosity distance applies only for spatially flat universes, which we are assuming throughout.

Furthermore, a very accurate and deep geometrical probe of dark energy is the angular scale of the sound horizon at the last scattering surface (i.e. at the time of decoupling of radiation from matter). The probe is described by the CMB "shift parameter" [65, 66] and is encoded in the location $l_{1}^{T T}$ of the first peak of the Cosmic Microwave Background (CMB) temperature perturbation spectrum. It provides the reduced distance to the last scattering surface. For spatially flat cosmologies it is given by

$$
R=\sqrt{\Omega_{m}^{0}} \int_{0}^{z_{*}} \frac{d z}{E(z)}
$$

The measured shift parameter according to the Planck data $[1,67]$ is $R=1.7499 \pm 0.0088$ at the redshift of decoupling (viz. at the last scattering surface), $z_{*}$. Its precise value depends weakly on the parameters, and it is obtained from the fitting formula [68]:

$$
z_{*}=1048\left[1+0.00124\left(\Omega_{b}^{0} h^{2}\right)^{-0.738}\right]\left[1+g_{1}\left(\Omega_{m}^{0} h^{2}\right)^{g_{2}}\right],
$$



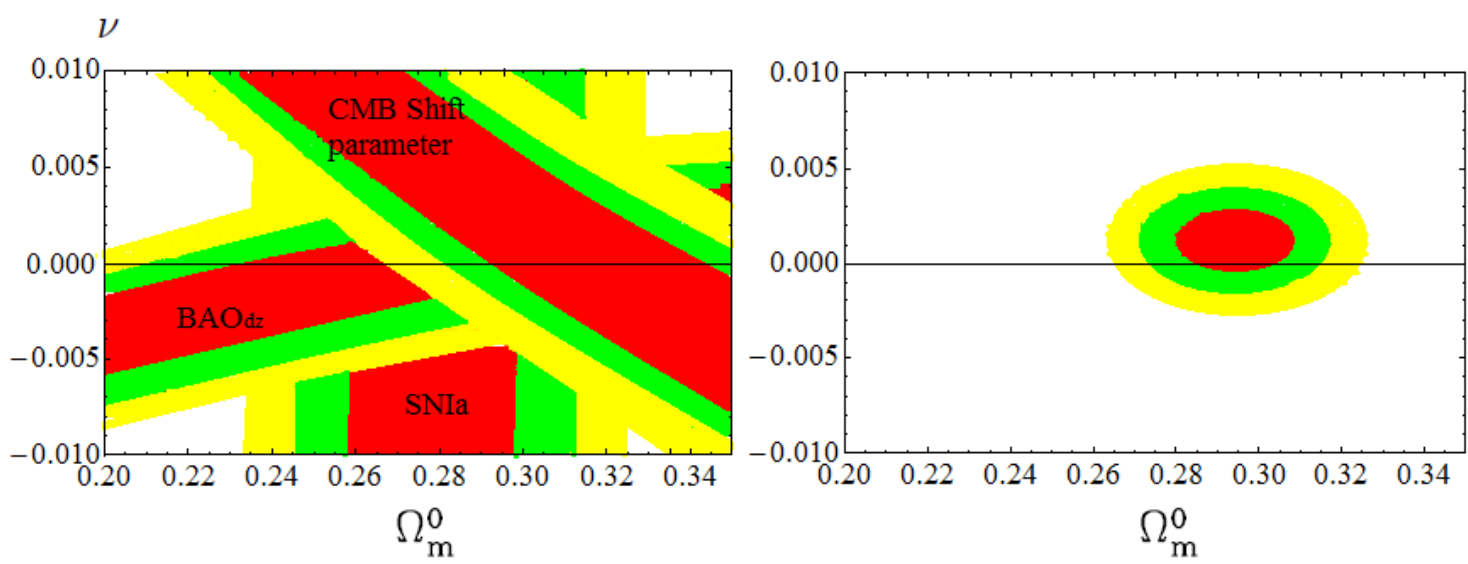

Figure 1: Likelihood contours (for $-2 \ln \mathcal{L} / \mathcal{L}_{\max }$ equal to $2.30,6.16$ and 11.81 , corresponding to $1 \sigma, 2 \sigma$ and $3 \sigma$ confidence levels) in the $\left(\Omega_{m}^{0}, \nu\right)$ plane for the A1 vacuum model $(\alpha \equiv 0)$. The left panel shows the contours based on the SNIa data (represented by approximate vertical bands), $\mathrm{BAO}_{d z}$ (diagonal bands) and CMB shift parameter (antidiagonal bands). On the right panel we show the corresponding contours based on the joint statistical analysis $\left(\mathrm{SNIa}+\mathrm{CMB}+\mathrm{BAO}_{d z}\right.$ data. From the inner to the outer regions (successively in red, green and yellow) we find the aforementioned $1 \sigma, 2 \sigma$ and $3 \sigma$ confidence levels, respectively.

with

$$
g_{1}=0.0783 \frac{\left(\Omega_{b}^{0} h^{2}\right)^{-0.238}}{1+39.5\left(\Omega_{b}^{0} h^{2}\right)^{0.763}}, \quad g_{2}=\frac{0.560}{1+21.1\left(\Omega_{b}^{0} h^{2}\right)^{1.81}} .
$$

In this case, the $\chi_{\mathrm{CMB}}^{2}$ function is given by:

$$
\chi_{\mathrm{CMB}}^{2}(\mathbf{p})=\frac{[R(\mathbf{p})-1.7499]^{2}}{0.0088^{2}} .
$$

As emphasized in the previous section, when dealing with the CMB shift parameter we have to include both the matter and radiation terms in the total normalized matter density entering the $E(z)$ function in (6.3) since the total radiation contribution at the last scattering amounts to some $\sim 23 \%$ of the total energy density associated to matter and is therefore not entirely negligible. It means that when we compute the CMB shift parameter we have to use the modified formulas for the Hubble function that we have found in Sect. 4.4 for type A and B models respectively.

Finally, we also consider the BAO scale produced in the last scattering surface by the competition between the pressure of the coupled baryon-photon fluid and gravity. The resulting acoustic waves leave (in the course of the evolution) an overdensity signature at certain length scales of the matter distribution. They appear as regular, periodic fluctuations of visible matter density in large-scale structure (LSS) resulting from sound waves propagating in the early Universe. Evidence of this excess has been found in the clustering properties of the SDSS galaxies (see $[69,70,71]$ ). In recent years, measurements of BAO have proven useful as a "standard ruler" or geometric probe that we can employ to constrain dark energy models. 

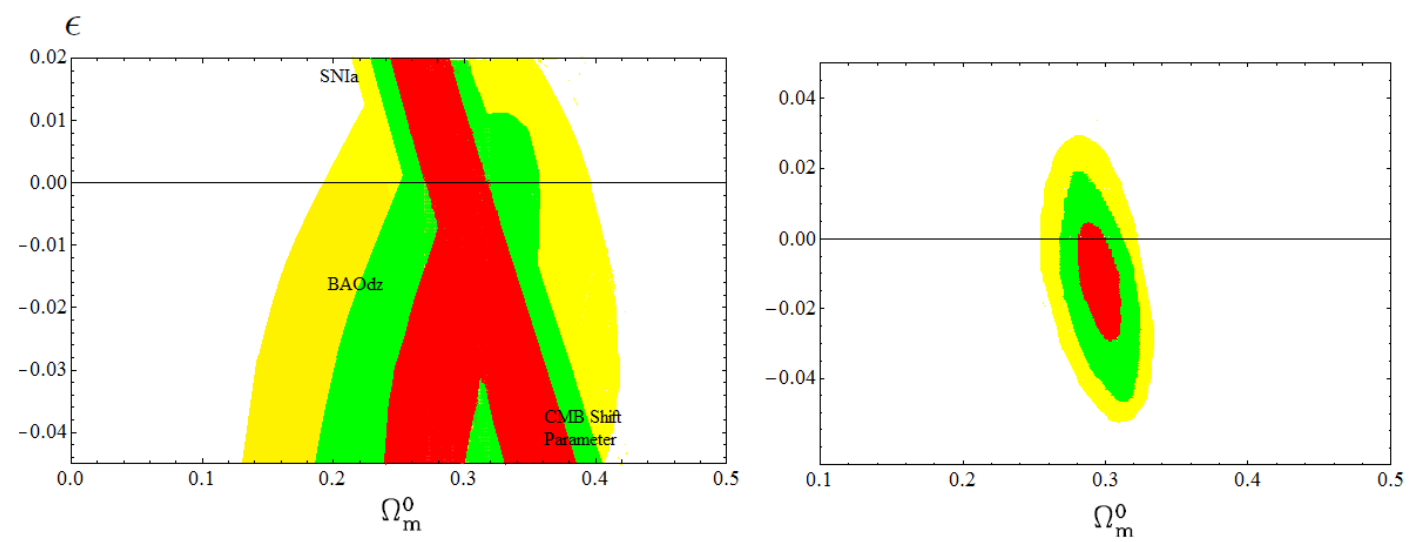

Figure 2: Likelihood contours for the $\mathrm{B} 1$ vacuum model, with $\mathrm{BAO}_{d z}$ data. The left panel shows the contours based on the SNIa data, $\mathrm{BAO}_{d z}$ and $\mathrm{CMB}$ shift parameter. The meaning of the shaded regions is as in Fig. 1. The various bands follow a similar pattern as in the previous figure, but here the overlapping of the SNIa and BAO regions is larger. The right panel shows the joint contours of $\mathrm{SNIa}+\mathrm{CMB}+\mathrm{BAO}_{d z}$.

In this work we use the results of Blake et al. [71] (cf. Table 3 of this reference) which are given in terms of the parameter $d_{z}\left(z_{i}\right)=r_{s}\left(z_{d}\right) / D_{\mathrm{V}}\left(z_{i}\right)$, where $D_{V}\left(z_{i}\right)$ is the effective distance measure [69] and $z_{i}$ is a reference redshift for observations. Moreover $r_{s}\left(z_{d}\right)$ is the comoving sound horizon size at the baryon drag epoch [72] (i.e. the epoch at which baryons are released from the Compton drag of photons), and $z_{d} \sim \mathcal{O}\left(10^{3}\right)$ is the corresponding redshift of that epoch, closely related to that of last scattering- the precise expression is given below, see Eq. (6.11).

Since $r_{s}\left(z_{d}\right)$ is the comoving distance that light can travel prior to redshift $z_{d}$, it can be computed as follows:

$$
r_{s}\left(z_{d}\right)=\int_{0}^{t\left(z_{d}\right)} \frac{c_{s} d t}{a}=\int_{0}^{a_{d}} \frac{c_{s}(a) d a}{a^{2} H(a)}=\int_{z_{d}}^{\infty} \frac{c_{s}(z) d z}{H(z)}
$$

where $a_{d}=\left(1+z_{d}\right)^{-1}$, and

$$
c_{s}(a)=c\left(\frac{\delta p_{\gamma}}{\delta \rho_{\gamma}+\delta \rho_{b}}\right)^{1 / 2}=\frac{c}{\sqrt{3(1+\mathcal{R}(a))}}
$$

is the sound speed in the baryon-photon plasma. Here we assume adiabatic perturbations and we have used $\delta p_{b}=0$ and $\delta p_{\gamma}=(1 / 3) \delta \rho_{\gamma}$, and defined $\mathcal{R}(a)=\delta \rho_{b} / \delta \rho_{\gamma}$. If the scaling laws for non-relativistic matter and radiation were those of the standard model, we would have $\mathcal{R}(a)=3 \rho_{b} / 4 \rho_{\gamma}$, which can be finally cast as $\mathcal{R}^{\Lambda C D M}(a)=\left(3 \Omega_{b}^{0} / 4 \Omega_{\gamma}^{0}\right) a$, where $\Omega_{b}^{0} h^{2} \simeq 0.02205$ and $\Omega_{\gamma}^{0} h^{2} \simeq 2.46 \times 10^{-5}$ are the current values of the normalized baryon and photon densities ${ }^{5}$. However, when we consider cosmologies beyond the $\Lambda$ CDM a modification of these formula for $\mathcal{R}(a)$ has to be implemented. We explain the details in the next section.

\footnotetext{
${ }^{5}$ We use $\Omega_{r}^{0}=4.153 \times 10^{-5} h^{-2}[67], \Omega_{\gamma}^{0}=\frac{\Omega_{r}^{0}}{1+0.2271 N_{\nu}}\left(\right.$ with $N_{\nu} \simeq 3.04$ and $\left.h=0.673[1]\right)$.
} 

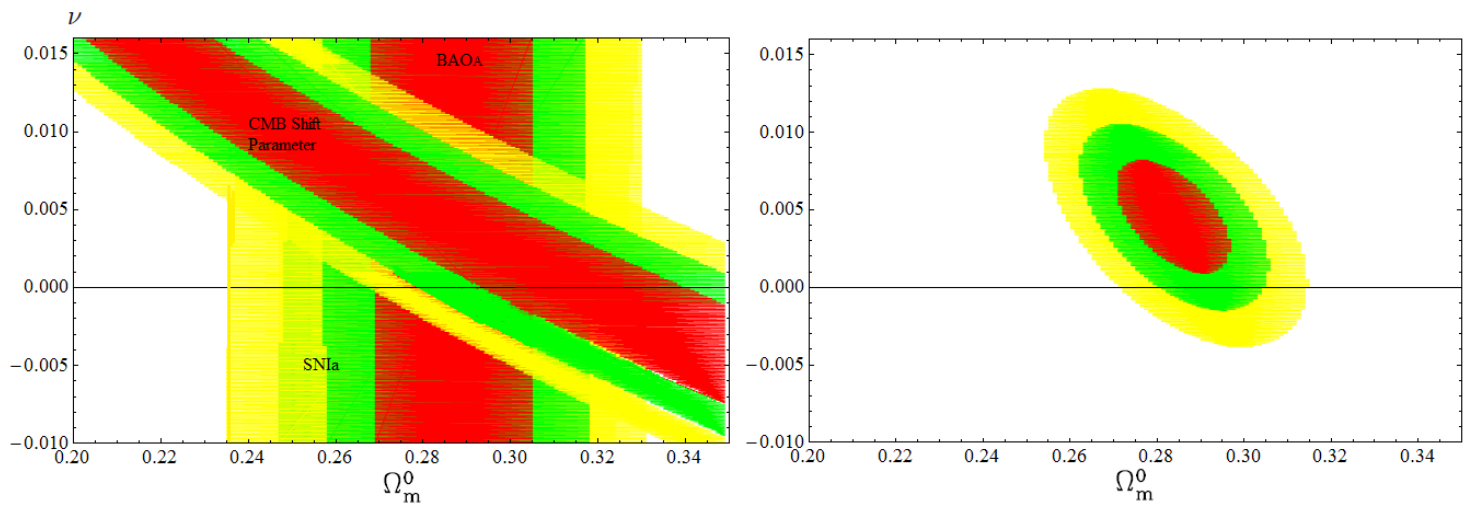

Figure 3: Likelihood contours for the $\mathrm{A} 1$ vacuum model, this time with $\mathrm{BAO}_{A}$ data. The left panel shows the contours based on the $\mathrm{SNIa}$ data, $\mathrm{BAO}_{A}$ and $\mathrm{CMB}$ shift parameter indicated in a similar way as in Fig. 1. The SNIa data and $\mathrm{BAO}_{A}$ bands appear as almost vertical and with significant overlap. The right panel shows the joint contours of $\mathrm{SNIa}+\mathrm{CMB}+\mathrm{BAO}_{A}$. A well defined final region is projected with $\nu>0$ at the $1 \sigma$ level.

The remaining ingredients of the $\mathrm{BAO}$ analysis are as in the standard case, in particular the effective distance is (see [69]):

$$
D_{\mathrm{V}}(z) \equiv\left[(1+z)^{2} D_{A}^{2}(z) \frac{c z}{H(z)}\right]^{1 / 3},
$$

where $D_{A}(z)=(1+z)^{-2} d_{L}(z, \mathbf{p})$ is the angular diameter distance. It follows from the foregoing that the $d_{z}$ estimator for BAO analysis explicitly reads:

$$
d_{z}\left(z_{i}\right)=\frac{r_{s}\left(z_{d}\right)}{\left[\left(\int_{0}^{z_{i}} \frac{c d z}{H(z)}\right)^{2}\left(\frac{c z_{i}}{H\left(z_{i}\right)}\right)\right]^{1 / 3}} .
$$

The fitted formula for the baryon drag redshift, $z_{d}$, is given by [72]:

$$
z_{d}=1291 \frac{\left(\Omega_{m}^{0} h^{2}\right)^{0.251}}{1+0.659\left(\Omega_{m}^{0} h^{2}\right)^{0.828}}\left[1+\beta_{1}\left(\Omega_{b}^{0} h^{2}\right)^{\beta_{2}}\right],
$$

with

$$
\beta_{1}=0.313\left(\Omega_{m}^{0} h^{2}\right)^{-0.419}\left[1+0.607\left(\Omega_{m}^{0} h^{2}\right)^{0.674}\right], \quad \beta_{2}=0.238\left(\Omega_{m}^{0} h^{2}\right)^{0.223} .
$$

As we see the drag epoch ends at a redshift which is somewhat more strongly dependent on the parameters than the decoupling redshift $z_{*}$. Numerically, $z_{d}$ is not very different from $z_{*}$, both being of order $10^{3}$ (with $z_{*}>z_{d}$ ). Typically $z_{*} \simeq 1090$ and $z_{d} \simeq 1060$ for the Planck results [1].

At this point, we would like to stress that Blake et al. [71] also provide BAO measurements in terms of the acoustic parameter $A(z)$, first introduced by Eisenstein et al. 

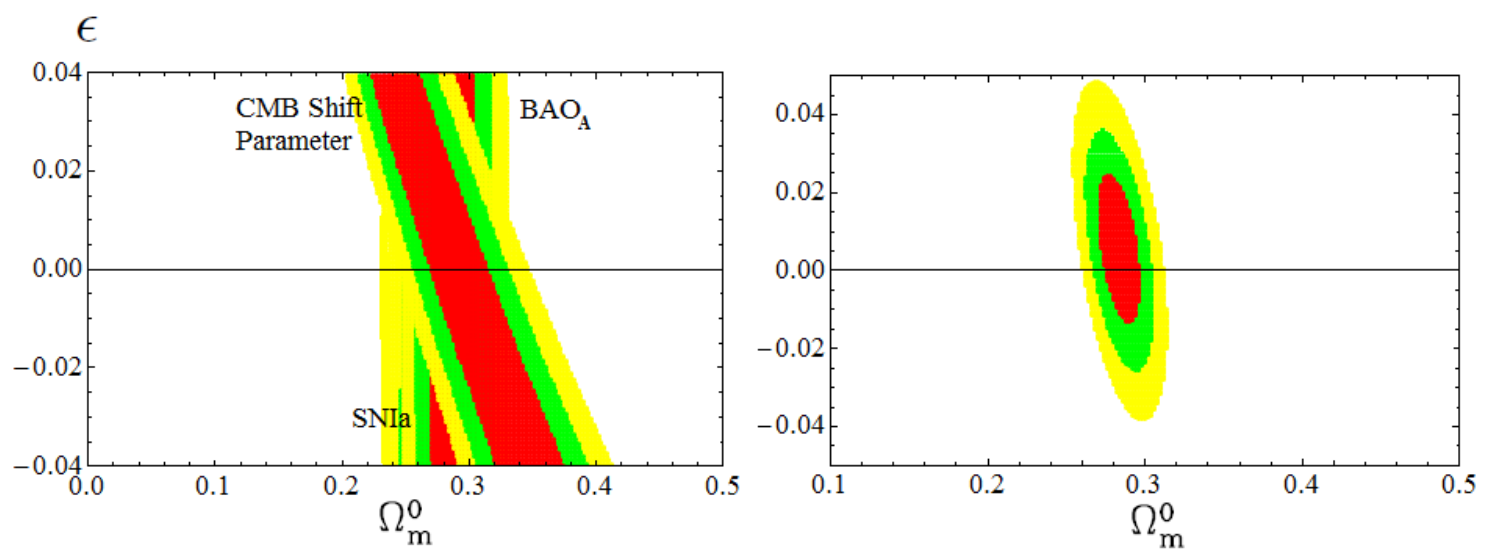

Figure 4: Likelihood contours for the $\mathrm{B} 1$ vacuum model, in this case with $\mathrm{BAO}_{A}$ data. The meaning of the shaded regions in the two panels is as in the previous figures.

[69]. Acoustic oscillations in the photon-baryon plasma prior to recombination give rise to a peak in the correlation function of galaxies, whose value is given by the mentioned $A(z)$-estimator for $\mathrm{BAO}$ analysis:

$$
A\left(z_{i}, \mathbf{p}\right)=\frac{\sqrt{\Omega_{m}^{0}}}{\left[z_{i}^{2} E\left(a_{i}\right)\right]^{1 / 3}}\left[\int_{a_{i}}^{1} \frac{d a}{a^{2} E(a)}\right]^{2 / 3}=\frac{\sqrt{\Omega_{m}^{0}}}{E^{1 / 3}\left(z_{i}\right)}\left[\frac{1}{z_{i}} \int_{0}^{z_{i}} \frac{d z}{E(z)}\right]^{2 / 3},
$$

with $a_{i}=\left(1+z_{i}\right)^{-1}$, and $z_{i}$ is the redshift at which the acoustic scale has been measured.

According to [71] the $A(z)$ measurements are approximately uncorrelated with respect to $\Omega_{m}^{0} h^{2}$, while this is not the case for the $d_{z}$ measurements. Therefore, it is natural to use both BAO estimators, $d_{z}$ and $A(z)$, in our statistical analysis (confer section 6.3) in order to check the range of validity of the free parameters included in the various vacuum models. Specifically, here and henceforth we consider the following two notations: $\mathrm{BAO}_{d z}$ for the $d_{z}$ measurements, and $\mathrm{BAO}_{A}$ for those based on the $A(z)$ estimator. Therefore, the corresponding $\chi^{2}$-functions for $\mathrm{BAO}$ analysis are defined as:

$$
\chi_{\mathrm{BAO} \mathrm{dz}}^{2}(\mathbf{p})=\sum_{i=1}^{6}\left[\frac{d_{z, \mathrm{th}}\left(z_{i}, \mathbf{p}\right)-d_{z, \mathrm{obs}}\left(z_{i}\right)}{\sigma_{z, i}}\right]^{2}
$$

and

$$
\chi_{\mathrm{BAO}_{\mathrm{A}}}^{2}(\mathbf{p})=\sum_{i=1}^{6}\left[\frac{A_{\mathrm{th}}\left(z_{i}, \mathbf{p}\right)-A_{\mathrm{obs}}\left(z_{i}\right)}{\sigma_{A, i}}\right]^{2},
$$

where $z_{i}, d_{z, \text { obs }}, \sigma_{z, i}, A_{\text {obs }}$ and $\sigma_{A, i}$ can be found in Table 3 of [71].

\subsection{Adapting the BAO analysis for dynamical vacuum models}

In this section we describe the necessary modifications to $\mathcal{R}(a)$ for the $\mathrm{BAO}_{d z}$ analysis when the cosmological vacuum is dynamical. The modifications are necessary since the 
scaling laws for non-relativistic matter and radiation are slightly different as compared to the $\Lambda$ CDM. For example, for type-A models the generalization is simple [21]. From the anomalous scaling laws (4.27) and (4.28) we easily find the following modification of the $\mathcal{R}^{\Lambda C D M}(a)$ function:

$$
\mathcal{R}^{\text {(typeA) }}(a)=\frac{3}{4} \frac{\xi}{\xi^{\prime}} \frac{\rho_{b}(a)}{\rho_{\gamma}(a)}=\frac{3}{4} \frac{1-4 \alpha / 3}{1-\alpha} \frac{\Omega_{b}^{0}}{\Omega_{\gamma}^{0}} a^{4 \xi^{\prime}-3 \xi} .
$$

Of course for $\nu=0$ and $\alpha=0\left(\xi=\xi^{\prime}=1\right)$ we recover $\mathcal{R}^{\Lambda C D M}(a)$ as given in the previous section.

For type-B models the corresponding $\mathcal{R}^{\Lambda C D M}(a)$ also differs from the standard one, but is more complicated. For this model we have to use the cosmic time rather than the scale factor, i.e. $R(t)=\delta \rho_{b}(t) / \delta \rho_{\gamma}(t)$. Thus, upon differentiating (4.34) and (4.35) with respect to $t$, and after some calculations we obtain:

$$
\mathcal{R}^{(\text {typeB })}(t)=\frac{3 \Omega_{b}^{0}}{4 \Omega_{\gamma}^{0}}\left[\frac{\sinh \left(\frac{3}{4} H_{0} \mathcal{F} t\right)}{\sinh \left(\frac{3}{4} H_{0} \mathcal{F} t_{0}\right)}\right]^{2 / 3} .
$$

One can easily check that for $\nu=\epsilon=0$, we have $\mathcal{F} \rightarrow 2 \sqrt{\Omega_{\Lambda}^{0}}$ and we recover the corresponding $\Lambda \mathrm{CDM}$ result:

$$
\left.\mathcal{R}^{(\text {typeB })}(t)\right|_{\epsilon=\nu=0}=\frac{3 \Omega_{b}^{0}}{4 \Omega_{\gamma}^{0}}\left[\frac{\sinh \left(\frac{3}{2} H_{0} \Omega_{\Lambda}^{0} t\right)}{\sinh \left(\frac{3}{4} H_{0} \Omega_{\Lambda}^{0} t_{0}\right)}\right]^{2 / 3}=\frac{3 \Omega_{b}^{0}}{4 \Omega_{\gamma}^{0}} a(t)=\mathcal{R}^{\Lambda C D M}(a) .
$$

Let us mention the following specification for fitting the $\mathrm{BAO}_{d z}$ observable (6.14) for type$\mathrm{B}$ models. In this case the computation of the parameter $r_{s}\left(z_{d}\right)$ - the comoving distance traveled by light to the drag epoch, Eq. (6.7) - is performed in two steps: in the first step we integrate from $a=0$ up to the decoupling (or last scattering) point $a_{*}$ by neglecting the vacuum energy corrections since dark energy effects are negligible for $a<a_{*}$; in the second step we integrate from $a=a_{*}$ to the drag epoch $a=a_{d}$ using the correction from the radiation component discussed above.

\subsection{Combined likelihood function}

In order to place tighter constraints on the corresponding parameter space of our model, the probes described above must be combined through a joint likelihood analysis ${ }^{6}$, given by the product of the individual likelihoods according to:

$$
\mathcal{L}_{\text {tot }}(\mathbf{p})=\mathcal{L}_{\mathrm{SNIa}} \times \mathcal{L}_{\mathrm{CMB}} \times \mathcal{L}_{\mathrm{BAO}}
$$

\footnotetext{
${ }^{6}$ Likelihoods are normalized to their maximum values. In the present analysis we always report $1 \sigma$ uncertainties on the fitted parameters. Note also that if the total number of data points is $N_{\text {tot }}$ the associated degrees of freedom is: $d o f=N_{\text {tot }}-n_{\text {fit }}$, where $n_{\text {fit }}$ is the model-dependent number of fitted parameters.
} 


\begin{tabular}{|c|c|c|c|c|c|}
\hline Model & $\Omega_{m}^{0}$ & $\nu$ & $\epsilon$ & $\chi^{2} / d o f$ & $\mathrm{AIC}$ \\
\hline$\Lambda \mathrm{CDM}$ & $0.293 \pm 0.013$ & - & - & $567.8 / 586$ & 569.8 \\
\hline$A 1$ & $0.292 \pm 0.014$ & $+0.0013 \pm 0.0018$ & - & $566.3 / 585$ & 570.3 \\
\hline$A 2$ & $0.290 \pm 0.014$ & $+0.0024 \pm 0.0024$ & - & $565.6 / 585$ & 569.6 \\
\hline$B 1$ & $0.297_{-0.014}^{+0.015}$ & - & $-0.014_{-0.013}^{+0.016}$ & $587.2 / 585$ & 591.2 \\
\hline$B 2$ & $0.300_{-0.003}^{+0.017}$ & $-0.0039_{-0.0021}^{+0.0020}$ & $-0.0039_{-0.0021}^{+0.0020}$ & $583.1 / 585$ & 587.1 \\
\hline
\end{tabular}

Table 1: The fit values for the various models using $\mathrm{SNIa}+\mathrm{CMB}+\mathrm{BAO}_{d z}$ data, together with their statistical significance according to $\chi^{2}$ and AIC statistical tests. Notice that for type-A2 models the quoted value of $\nu$ stands actually for $\nu_{\text {eff }}$ under the conditions explained in the text, and for B2 we have set $\nu=\epsilon$ (see the text as well).

This translates into an addition of the joint $\chi^{2}$ function:

$$
\chi_{\mathrm{tot}}^{2}(\mathbf{p})=\chi_{\mathrm{SNIa}}^{2}+\chi_{\mathrm{CMB}}^{2}+\chi_{\mathrm{BAO}}^{2},
$$

where $X$ denotes the kind of BAO measurements used in the statistical analysis, namely $X=d_{z}$ or $X=A(z)$. In our $\chi^{2}$ minimization procedure, for the vacuum models (running and concordance $\Lambda \mathrm{CDM}$ ) we use the following range and steps of the fitted parameters: $\Omega_{m}^{0} \in[0.1,1]$ in steps of 0.001 and $\nu \in[-0.02,0.02]$ in steps of $10^{-4}$.

Since the current vacuum models contain different number of free parameters, as a further statistical test we use the (corrected) Akaike Information Criterion (AIC) relevant to our case $\left(N_{\text {tot }} / n_{\text {fit }}>40\right)$ [73], which is defined, for the case of Gaussian errors, as follows:

$$
\mathrm{AIC}=\chi_{\mathrm{tot}}^{2}+2 n_{f i t} .
$$

It is well known that a smaller value of AIC points to a better model-data fit. In this context, we have to mention that small differences in AIC are not necessarily significant and therefore, in order to test the effectiveness of the models themselves, it is important to calculate the model pair difference $\Delta \mathrm{AIC}=\mathrm{AIC}_{y}-\mathrm{AIC}_{x}$. The larger the value of $|\Delta \mathrm{AIC}|$, the higher the evidence against the model with larger value of AIC, with a difference $|\Delta \mathrm{AIC}| \geq 2$ indicating a positive such evidence and $|\Delta \mathrm{AIC}| \geq 6$ indicating a strong such evidence, while a value $\leq 2$ indicates consistency among the two comparison models.

Let us next present the basic results of the overall statistical analysis.

1. We first consider $S N I a+C M B+B A O_{d z}$.

- In the case of the concordance $\Lambda$ CDM cosmology we find $\Omega_{m}^{0}=0.293 \pm 0.013$ with statistic significance $\chi_{\text {tot }}^{2}\left(\Omega_{m}^{0}\right) / d o f \simeq 567.8 / 586\left(\mathrm{AIC}_{\Lambda} \simeq 569.8\right)$. For comparison, the determination by Planck $+\mathrm{WP}[1]$ reads: $\Omega_{m}^{0} h^{2}=0.1426 \pm 0.0025$. With $h=0.673 \pm 0.012$ (also from the same source) this yields $\Omega_{m}^{0} \simeq 0.315 \pm 0.016$, which agrees with our value within slightly more than $1 \sigma$. We shall comment 
further on the possible implications of the $\Omega_{m}^{0}$ determination in the next section. Another important cosmological parameter that will enter later our analysis is the rms mass fluctuation on $R_{8}=8 h^{-1} \mathrm{Mpc}$ scales at $z=0$. In this work we use as a prior for such parameter the value $\sigma_{8, \Lambda}=0.829$ in the $\Lambda$ CDM, i.e. the Planck + WP result [1]. Then, with the aid of this value, we can calculate the corresponding $\sigma_{8}$ values for the vacuum models through Eq.(7.10) below [see discussion in section 7.1 and Tables 3 and 4$]$.

- In Fig. 1 we present the results of our analysis for the type-A1 running vacuum model, which is characterized by the $\nu$-parameter. We have sampled this parameter in the interval $[-0.02,0.02]$ in steps of $10^{-4}$. The left panel in that figure shows the fitted regions at $1 \sigma, 2 \sigma$ and $3 \sigma$ confidence levels in the $\left(\Omega_{m}^{0}, \nu\right)$ plane, from the SNIa, $\mathrm{BAO}_{d z}$ and $\mathrm{CMB}$ shift parameter data. The right panel in the figure shows the fit contours when the three types of data are intersected. Using the SNIa data alone it is evident (from the left panel) that although the $\Omega_{m}^{0}$ parameter is tightly constrained $(\sim 0.29)$, the $\nu$ parameter remains completely unconstrained (in the shown interval). However, as it is manifest from the right panel of that figure, the above degeneracy is broken when we use the joint likelihood analysis with all the cosmological data. Indeed the overall likelihood function peaks at $\Omega_{m}^{0}=0.292 \pm 0.014$ and $\nu=+0.0013 \pm 0.0018$ with $\chi_{\text {tot }}^{2}\left(\Omega_{m}^{0}, \nu\right) \simeq 566.3\left(\mathrm{AIC}_{A 1} \simeq 570.3\right)$ for 585 degrees of freedom ${ }^{7}$. The $\Lambda \mathrm{CDM}$ value of $\mathrm{AIC}(\simeq 569.8)$ is smaller with respect to that of the A1 model. This indicates that the fit of the concordance model to the combined data is slightly better than that of the A1 vacuum model. However, the differential AIC value $|\Delta \mathrm{AIC}|=\left|\mathrm{AIC}_{\Lambda}-\mathrm{AIC}_{A 1}\right|=1.5$ is actually $\leq 2$. This tells us that the cosmological data are perfectly consistent with the A1 model in a way comparable to the $\Lambda$ CDM. Furthermore, we find that $\sigma_{8}=0.813$.

- Let us now address the more general case of the type-A2 model,whose statistical vector $\mathbf{p}$ contains 3 free parameters, namely $\mathbf{p}=\left(\Omega_{m}^{0}, \nu, \alpha\right)$. Our minimization analysis provides strongly degenerate results between $\nu$ and $\alpha$, rendering impossible to put any significant constraints on their values ${ }^{8}$. Note, however that the value of $\Omega_{m}^{0}$ is well constrained $(\simeq 0.29)$. Therefore we adopt - as in Ref. [21] - the additional setting $\xi^{\prime} \equiv 1$, which is tantamount to assume that there is no modification in the scaling law of radiation and hence radiation behaves in the strict standard way, in contraposition to dust. This occurs when

\footnotetext{
${ }^{7}$ Note that in [20] we used the earlier BAO results of Percival [70] and the Constitution set of 397 SNIa [74]. We would like to mention here that those results are in agreement with the current results within $1 \sigma$ uncertainties.

${ }^{8}$ This is similar to the situation with the CPL parameterization [75] of the dark energy, where in the statistical vector $\mathbf{p}=\left(\Omega_{m}^{0}, w_{0}, w_{1}\right)$ one actually has to fix a parameter to fit the other two in an efficient way
} 
$\alpha=\frac{3}{4} \nu$ (see equation 4.26), implying from Eq.(4.11) that $\xi \simeq 1-\nu_{\text {eff }}$, in which $\nu_{\text {eff }} \simeq(\nu-\alpha)=\nu / 4$. The statistical vector reduces in this case to $\mathbf{p}=\left(\Omega_{m}^{0}, \nu_{\text {eff }}\right)$, and we sample $\nu_{\text {eff }} \in[-0.02,0.02]$ in steps of $10^{-4}$. The joint minimization provides now $\Omega_{m}^{0}=0.29 \pm 0.014, \nu_{\mathrm{eff}}=0.0024 \pm 0.0024(\nu \simeq 0.0096$ and $\alpha \simeq 0.0072)$ with $\chi_{\text {tot }}^{2}\left(\Omega_{m}^{0}, \nu_{\text {eff }}\right) / d o f \simeq 565.6 / 585$ and $\mathrm{AIC}_{A 2} \simeq 569.6$. Notice that in the present case, opposite to the A1 model, the fit to the combined data from the A2 model is slightly better than in the $\Lambda$ CDM model (cf. Table 1). However, utilizing the $\mathrm{AIC}$ information criterium, and because $\mathrm{AIC}_{\Lambda} \simeq \mathrm{AIC}_{A 2}$ we have that the $\mathrm{A} 2$ vacuum model is statistically equivalent $(|\Delta \mathrm{AIC}| \leq 2)$ with the $\Lambda \mathrm{CDM}$ model. In this case we obtain $\sigma_{8}=0.797$.

- We next face model $\mathrm{B} 1$ with $\mathrm{BAO}_{d z}$ data. As in the A1 model, here we have only one characteristic parameter, in this case $\epsilon$ (apart from the generic one $\Omega_{m}^{0}$ ). We have dealt with the fitting procedure of $\epsilon$ in a similar way as $\nu$ for the A1 model. The fitting regions in the $\left(\Omega_{m}^{0}, \epsilon\right)$-plane are shown in Fig. 2, left panel, whereas the $1 \sigma, 2 \sigma$ and $3 \sigma$ contour lines for the combined $\mathrm{SNIa}+\mathrm{CMB}+\mathrm{BAO}_{d z}$ data are displayed on the right panel of that figure. As we can see, the determination of $\Omega_{m}^{0}$ is rather sharp around 0.3 , to be precise: $\Omega_{m}^{0}=0.297_{-0.014}^{+0.015}$. However the parameter $\epsilon$ is not so well bounded by the data as it was the case of $\nu$, specifically we find $\epsilon=-0.014_{-0.013}^{+0.016}$. The central value and the errors are roughly one order of magnitude bigger than before, and the fit quality is significantly poorer. This is clear from the $\chi^{2} / d o f$ and AIC statistical diagnostics in Table 1, which give substantially larger values than those in the $\Lambda \mathrm{CDM}$ model. If we attend strictly the AIC statistical criterion we should conclude that the type-B1 model does not fit at all the combined data in a way comparable to type-A1 or A2 models and the $\Lambda$ CDM. Concerning the rms mass fluctuation for this model, we find $\sigma_{8}=0.859$.

- As for the B2 model, we have once more a situation with three fit parameters $\left(\Omega_{m}^{0}, \nu, \epsilon\right)$. But to avoid similar difficulties as those mentioned with the A2 model, we fix a correlation between the two model parameters $\nu$ and $\epsilon$. Of course, both are small in absolute value, but let us note that for $\nu \ll \epsilon$ model B2 must reduce to B1, whereas for $\epsilon \ll \nu$ it essentially behaves as A1. Therefore, the parameter region which is left unexplored is when $\epsilon \simeq \nu$, and for definiteness we will fix $\epsilon=\nu$ and shall perform a two-parameter fit in $\left(\epsilon, \Omega_{m}^{0}\right)$. Under these conditions we find $\epsilon \simeq-0.0039$ and $\Omega_{m}^{0} \simeq 0.30$ (cf. Table 1 for more precise values and errors). As with the B1 case, the quality of the fit is worse than for the $\Lambda \mathrm{CDM}$ or any of the type-A models. Moreover, for the $\mathrm{B} 2$ model, we find $\sigma_{8}=0.896$.

2. In the following we are based on $S N I a+C M B+B A O_{A}$ data. 
- We describe now the situation concerning the various models for when we use the alternative BAO option. The corresponding results are clearly displayed in Table 2 .

For the $\Lambda$ CDM model we obtain $\Omega_{m}^{0}=0.292 \pm 0.011$ with $\chi_{\text {tot }}^{2}\left(\Omega_{m}^{0}\right) / d o f \simeq$ $567.5 / 586\left(\mathrm{AIC}_{\Lambda} \simeq 569.5\right)$. As it could be expected, we obtain almost the same results with those of the previous fit with $\mathrm{SNIa}+\mathrm{CMB}+B A O_{d z}$ data.

- A1 vacuum model: Here the overall likelihood function peaks at a lower value of the mass parameter $\Omega_{m}^{0}=0.282 \pm 0.012$ (see the concluding comments of this section) and higher running parameter $\nu=0.0048_{-0.0031}^{+0.0032}$, with $\chi_{\text {tot }}^{2}\left(\Omega_{m}^{0}, \nu\right) \simeq$ $563.8\left(\mathrm{AIC}_{A 1} \simeq 567.8\right)$ for 585 degrees of freedom. The shape of the $\mathrm{BAO}_{A^{-}}$ contours in Fig. 3 are quite different from those of $\mathrm{BAO}_{d z}$ (Fig. 1). This is somehow related with the fact that unlike for the case of $A(z)$, the $d_{z} \mathrm{BAO}$ measurements are correlated with the $\Omega_{m}^{0} h^{2}$ [71] as well as with the necessary modifications to $\mathcal{R}(a)$ introduced in the $\mathrm{BAO}_{d z}$ analysis (see section 7.2). The rms mass fluctuation is found to be $\sigma_{8}=0.758$. Let us note the remarkable fact that using the $\mathrm{BAO}_{A}$ observable, instead of $\mathrm{BAO}_{d z}$, the value of $\nu$ is not compatible with zero at $1 \sigma$, showing a slight tendency to favor nonvanishing values of $\nu$ rather than the $\Lambda \mathrm{CDM}$ result.

- A2 vacuum model: the overall minimization provides $\Omega_{m}^{0}=0.283 \pm 0.012, \nu_{\text {eff }}=$ $+0.0048 \pm 0.0031(\nu \simeq 0.019$ and $\alpha \simeq 0.014)$ with $\chi_{\text {tot }}^{2}\left(\Omega_{m}^{0}, \nu_{\text {eff }}\right) / d o f \simeq 563.8 / 585$ and $\mathrm{AIC}_{A 2} \simeq 567.8$. The rms mass fluctuation is $\sigma_{8}=0.757$.

At this point we would like to make some comments for the A1-A2 model. Generally, the $\Omega_{m}^{0}$ values are in agreement (with $1 \sigma$ errors) with those of SNIa+CMB+ $\mathrm{BAO}_{d z}$. However, as far as $\nu_{\text {eff }}$ (or $\nu$ ) is concerned we find differences among the parameters which could reach up to a factor of $\sim 3.7$. In this context, the $\mathrm{SNIa}+\mathrm{CMB}+\mathrm{BAO}_{A}$ data analysis highlights the fact that the values of $\mathrm{AIC}_{A 1-A 2}(\simeq 567.8)$ are actually smaller with respect to those of the concordance $\Lambda \mathrm{CDM}$ cosmology. In other words, it turns out that the type-A1 and A2 vacuum models appear now to fit slightly better than the $\Lambda \mathrm{CDM}$ the observational data. Still, the $|\Delta \mathrm{AIC}|=\left|\mathrm{AIC}_{A 1-A 2}-\mathrm{AIC}_{\Lambda}\right|$ values (ie., $\leq 2$ ) indicate that the cosmological data are simultaneously consistent with the $\mathrm{A} 1, \mathrm{~A} 2$ and the $\Lambda$ CDM models.

- B1 model with $\mathrm{BAO}_{A}$ data. We find $\Omega_{m}^{0}=0.283_{-0.011}^{+0.012}$, so it remains similar to the type-A models, with $\epsilon=+0.005_{-0.015}^{+0.018}$. Interestingly, the fit quality is also in this case slightly better than in the $\Lambda \mathrm{CDM}$ model, but still with $|\Delta \mathrm{AIC}| \leq 2$, and hence statistically comparable. The rms mass fluctuation is $\sigma_{8}=0.820$.

- For the B2 model we proceed here with a similar strategy as with the $\mathrm{BAO}_{d z}$ case, and we find $\Omega_{m}^{0}=0.283$ and $\epsilon \simeq \nu \simeq+0.0015$. The quality of the fit 


\begin{tabular}{r|c|c|c|c|c|}
\multicolumn{1}{r}{ Model } & \multicolumn{2}{c}{$\nu$} & $\epsilon$ & $\chi^{2} /$ dof & \multicolumn{1}{c|}{ AIC } \\
\hline$\Lambda \mathrm{CDM}$ & $0.292 \pm 0.011$ & - & - & $567.5 / 586$ & 569.5 \\
\hline$A 1$ & $0.282 \pm 0.012$ & $+0.0048_{-0.0031}^{+0.0032}$ & - & $563.8 . / 585$ & 567.8 \\
\hline$A 2$ & $0.283 \pm 0.012$ & $+0.0048 \pm 0.0031$ & - & $563.8 / 585$ & 567.8 \\
\hline$B 1$ & $0.283_{-0.011}^{+0.012}$ & - & $+0.005_{-0.015}^{+0.018}$ & $563.7 / 585$ & 567.7 \\
\hline$B 2$ & $0.283_{-0.012}^{+0.011}$ & $+0.0015_{-0.0030}^{+0.0025}$ & $+0.0015_{-0.0030}^{+0.0025}$ & $563.8 / 585$ & 567.8 \\
\hline$C 1$ & $0.296 \pm 0.017$ & $-0.189 \pm 0.008$ & - & $568.3 / 585$ & 570.3 \\
\hline
\end{tabular}

Table 2: The fit values for the various models using the same data and statistical tests as before except for the $\mathrm{BAO}$ observable, which now is $\mathrm{BAO}_{A}$, i.e., overall we use $\mathrm{SNIa}+\mathrm{CMB}+\mathrm{BAO}_{A}$ data. In this case we include also the $\mathrm{C} 1$ model (see text). Same notation as the previous table.

is once more comparable, but better, than for the concordance $\Lambda \mathrm{CDM}$ model. The rms mass fluctuation is $\sigma_{8}=0.791$.

- Finally, the observational viability of the $\mathrm{C} 1$ model has been tested previously in Basilakos \& Solà [23], and thus for the rest of the paper we use their $\mathrm{SNIa}+\mathrm{CMB}+\mathrm{BAO}_{A}$ analysis of the $\left(\Omega_{m}^{0}, \zeta\right)$ pair. Specifically, we remind the reader that the following results were found [23]: $\Omega_{m}^{0}=0.296 \pm 0.017$, $\zeta=1.189 \pm 0.008$ with $\chi_{\text {tot }}^{2}\left(\Omega_{m}^{0}, \zeta\right) / d o f \simeq 568.3 / 585$. Recall that for C1 models $\zeta=1-\nu$, and the fitted value of $\nu$ is the one indicated for this model in Table 2. Because of the absence of the constant additive term for the $\mathrm{C} 1$ model (cf. Sect. 4.3), the value of $\nu$ is forced to be much larger than in the other models. Notwithstanding, the corresponding AIC value is 570.3 , which is statistically comparable to that of the $\Lambda \mathrm{CDM}$ model, and therefore at least from the point of view of the Hubble expansion data and the shift parameter, the $\mathrm{C} 1$ model seems to present a respectable status. But it is only an ostensible good status. The situation for this model will undergo a radical change when we test the structure formation data at low redshifts, as we shall see in the next section. The trouble is related to the aforementioned absence of the additive term. A first hint of decline of this model appears when we compute the corresponding rms mass fluctuation, namely $\sigma_{8}=1.365$, which is clearly anomalously large.

We conclude this section with the following observation. The fitting functions (6.4) and (6.11) for the redshifts of decoupling and baryon drag epochs respectively, have been obtained $[68,72]$ under the assumption that the precise scaling laws for matter are as in the $\Lambda \mathrm{CDM}$ concordance model. This is not the case for our dynamical vacuum models, see e.g. (4.27) and (4.28). Therefore it is natural to assess if this can have a significant effect in our analysis. In regard to Eq. (6.4) the small deviations around the standard value of $z_{*}$ caused by the anomalous scaling laws in the natural region (4.10) do not affect significantly the value of the integral (6.3) (the shift parameter). As for the baryon drag 
redshift $z_{d}$ we have roughly estimated the possible effects by considering the method of Ref. [76] in combination with the framework of [68]. In this way we can test the sensibility of our fits to the vacuum models under study, with and without the scaling correction on the $R$-functions of the BAO analysis, i.e. equations (6.16) and (6.17), which enter the determination of $z_{d}$ with the method of [76] (cf. Appendix A of this reference). While we have obtained some differences, in all cases they are compatible with the parameter errors indicated in Tables 1 and 2. Let us also notice that these corrections can only affect the $\mathrm{BAO}$ data associated to the $d_{z}$ observable (6.10), but not the BAO analysis based on the $A(z)$ estimator (6.13). If in the future more accurate cosmological data becomes available, one may have to consider the effect of these corrections for a better determination of the parameters potentially responsible for the vacuum dynamics.

\subsection{Discussion of the fitting results and implications for dynamical DE}

From the previous analysis one could tentatively say that type-A models are preferred to type- $\mathrm{B}$ ones from the point of view of the quality fits to the combined data. This is indeed suggested by the results involving $\mathrm{BAO}_{d z}$. In contrast, $\mathrm{BAO}_{A}$ data does not seem to point so strongly to this conclusion. This is somehow understandable if we take into account that the $\mathrm{BAO}_{A}$ data are exclusively based on the imprints of baryonic acoustic oscillations left at low redshifts during the early epochs of galaxy clusters formation, which means at relatively recent times, whereas the $\mathrm{BAO}_{d z}$ data is also sensitive to the model behavior of these oscillations at earlier epochs in between the decoupling and baryon drag epochs. More observational work will be necessary to decide about the best vacuum models.

We come now to a point mentioned in passing in the previous section concerning the fitting values of $\Omega_{m}^{0}$. For the $\Lambda$ CDM we have found $\Omega_{m}^{0} \simeq 0.293$ (virtually independent of the type of BAO data used). This is smaller than the Planck+WP value $\Omega_{m}^{0} \simeq 0.315$. Similarly, for the vacuum models $\mathrm{A}$ and $\mathrm{B}$ we have found $\Omega_{m}^{0}$ smaller than the Planck+WP value. This holds not only for $\mathrm{BAO}_{d z}$ data (cf. Table 1) but even more pronounced when the fit is done using $\mathrm{BAO}_{A}$ data, where the value of $\Omega_{m}^{0}$ lessens significantly for all vacuum models at around $\Omega_{m}^{0} \simeq 0.282-0.283$ (cf. Table 2 ). At the same time one obtains, in the last case, a slightly improved fit quality with respect to the $\Lambda \mathrm{CDM}$ for all the dynamical vacuum models $\mathrm{A}$ and $\mathrm{B}$. The difference with respect to the Planck+WP value of $\Omega_{m}^{0}$ is now larger and, as we will see in Sect.7, it does matter as far as the possible implications on the predicted cluster number counts for the dynamical models. At this stage of precision cosmology it is difficult to make a final selection between the two types of BAO data, and therefore we have decided to present the results separately for each BAO set.

The importance of the BAOs measurements cannot be underemphasized. They are sensitive to the physics of large scales and hinge primarily on the well-known principles of the linear regime of gravitational instability. Recall the recent hint of dynamical dark energy based on BAOs data mentioned in the introduction - cf. Ref. [33]. These authors 
utilize the "Om(z)-diagnostic" [77] for dark energy from the recent measurement of $H(z)$ made, in particular, on the basis of BAOs in the Ly $\alpha$ forest of BOSS DR11 quasars [79]. Such DE diagnostic is defined as

$$
O m(z) \equiv \frac{E^{2}(z)-1}{(1+z)^{3}-1} .
$$

For the $\Lambda \mathrm{CDM}$ one finds the constant value $\operatorname{Om}(z)=\Omega_{m}^{0}$. According to the test, a departure from this result points to dynamical vacuum energy. A related test is the twopoint diagnostic [33] defined as follows. Introducing $h(z)=E(z) h$ (where $h$ is the reduced Hubble parameter), the test is based on the quantity

$$
O m h^{2}\left(z_{2}, z_{1}\right) \equiv \frac{h^{2}\left(z_{2}\right)-h^{2}\left(z_{2}\right)}{\left(1+z_{2}\right)^{3}-\left(1+z_{1}\right)^{3}} .
$$

It has the following properties. To start with, $O m h^{2}(z, 0)=O m(z) h^{2}$. In addition, for the $\Lambda \mathrm{CDM}$ we have $O m h^{2}\left(z_{2}, z_{1}\right)=\Omega_{m}^{0} h^{2}$, which is constant for any pair of points $z_{2}$ and $z_{1}$. Using the previous diagnostic and the known observational information on $H(z)$ at the three redshift values $z=0,0.57,2.34$ (the last one being from [79]) the authors of [33] observe that the average result is: $O m h^{2}=0.122 \pm 0.01$, with very little variation from any pair of points taken. The obtained result is significantly smaller than the corresponding Planck+WP value of the two-point diagnostic, which is obviously constant and given by $O m h^{2}=\Omega_{m}^{0} h^{2}=0.1426 \pm 0.0025$. Since a departure of $O m h^{2}$ from this result should, according to [33], signal that the DE is not the $\Lambda$-cosmology, the obvious tension found between the $\mathrm{BAO}$ observations and the $\mathrm{CMB}$ measurements (assuming the concordance $\Lambda \mathrm{CDM}$ model) cannot be explained within the $\Lambda \mathrm{CDM}$ model.

The above mentioned authors make a case for this result and conclude that the spotted difference provides model-independent evidence for dynamical DE. Although it is probably too early to draw definite conclusions from these results before we get more statistics on $H(z)$ at high redshifts (cf. e.g. [78]), we can at least say that this kind of scenario is roughly consistent with the results of the current analysis. We have indeed found that our vacuum dynamical framework, when confronted with the presently available SNIa+CMB+BAO data, tends to emphasize significantly smaller values of $\Omega_{m}^{0}$. Therefore, in case that the claims of dynamical DE would be confirmed at some point, the vacuum models presented here could provide an explanation.

We can understand analytically the possible origin of these results in our theoretical framework. Let us take e.g. a general type-A model. From the formulae of Sect.4.1 we can easily compute the corresponding $O m(z)$-diagnostic. The result is:

$$
O m h^{2}(z)=\frac{\Omega_{m}^{0}}{\xi} \frac{(1+z)^{3 \xi}-1}{(1+z)^{3}-1}
$$

with $\xi$ given in Eq. (4.4). It is pretty obvious that for $\xi=1$ we recover the $\Lambda$ CDM result, which remains pegged to $\operatorname{Om}(z)=\Omega_{m}^{0}(\forall z)$. However, as soon we allow a small dynamical 


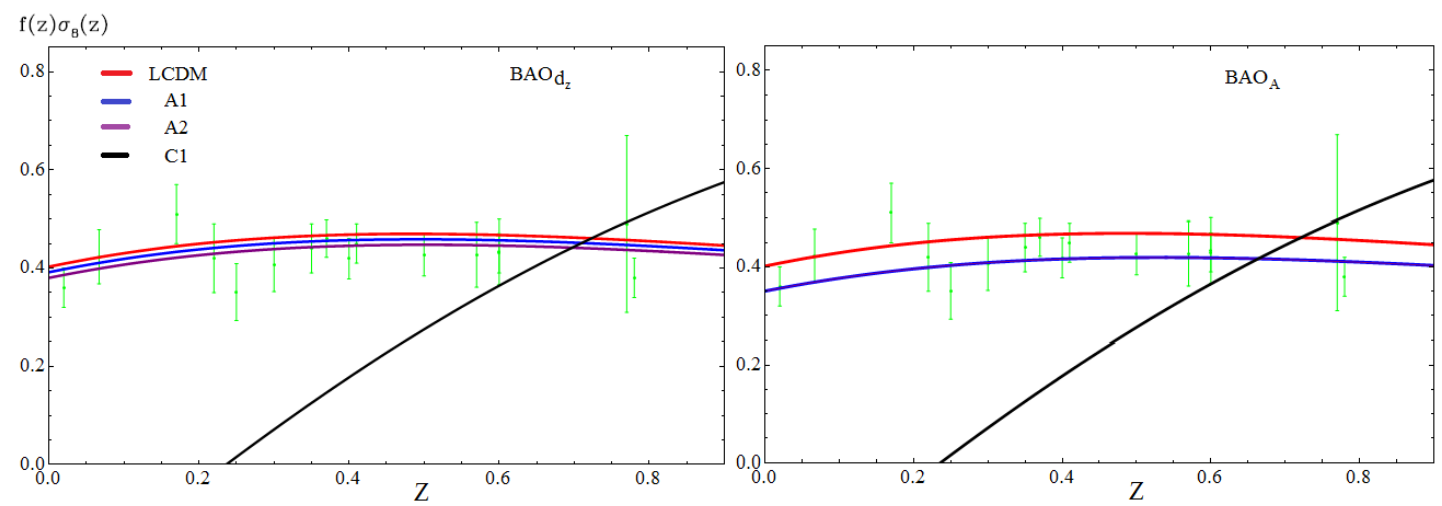

Figure 5: Comparison of the observed (solid points with vertical error bars) and theoretical evolution of the weighted growth rate $f(z) \sigma_{8}(z)$ for the models $\mathrm{A} 1, \mathrm{~A} 2$ and $\mathrm{C} 1$. The uppermost (red) line corresponds to the $\Lambda$ CDM model, used as a reference. The subsequent ones (from top to bottom) correspond to the vacuum models A1 (blue line) and A2 (purple line). On the right panel, the lines for A1 and A2 are one essentially overlapping. The curve that deviates significantly from the others in the two panels and loses power quickly near our time corresponds to model C1 (black line). The curves have been obtained for the best fit values of the cosmological parameters as discussed in Sec. 6.3 (for a summary, cf. Tables 1 and 2). The left panel shows the results based on the $\mathrm{SNIa}+\mathrm{CMB}+\mathrm{BAO}_{d z}$ fitting while the right panel those of the $\mathrm{SNIa}+\mathrm{CMB}+\mathrm{BAO}_{A}$ analysis. The $\mathrm{C} 1$ curve is obtained only for $\mathrm{SNIa}+\mathrm{CMB}+\mathrm{BAO}_{A}$ data (and therefore is the same in both panels).

running of vacuum (meaning $\nu$ and or $\alpha$ different from zero) we obtain a small departure of $\xi$ from 1 and therefore the DE diagnostic $\operatorname{Om}(z)$ deviates from $\Omega_{m}^{0}$. Actually, in this case (6.24) evolves with time (or redshift). According to the $\operatorname{Om}(z)$ diagnostic this implies that the vacuum energy is dynamical. By the same token, the two-point diagnostic for type-A models can be computed:

$$
O m h^{2}\left(z_{2}, z_{1}^{2}\right)=\frac{\Omega_{m}^{0}}{\xi} \frac{\left(1+z_{2}\right)^{3 \xi}-\left(1+z_{1}\right)^{3 \xi}}{\left.\left(1+z_{2}\right)^{3}-1+z_{1}\right)^{3}}
$$

Clearly, the result depends on $z_{i}$ for $\xi \neq 1$. Only for the $\Lambda$ CDM case $(\xi=1)$ it remains anchored to $\Omega_{m}^{0} h^{2}$ for any $z_{i}$. Similar considerations hold for type-B models. The upshot is that the detailed numerical analysis of both model types of dynamical vacuum models, in the light of the available observations, confirms that such vacuum dynamics leads to smaller $\Omega_{m}^{0}-$ cf. Sect. 6.3.

\subsection{The linear growth rate of clustering and the $\gamma$ index}

In this section we analyze the linear perturbation growth regime for the various models. Although one could do it by means of the power spectrum, we follow the approach of [19] and will test herein the implications of the various models on structure formation through the study of the linear growth rate of clustering [38]. This important (dimensionless) indicator is defined as the logarithmic derivative of the linear growth factor $D(a)$ with respect 


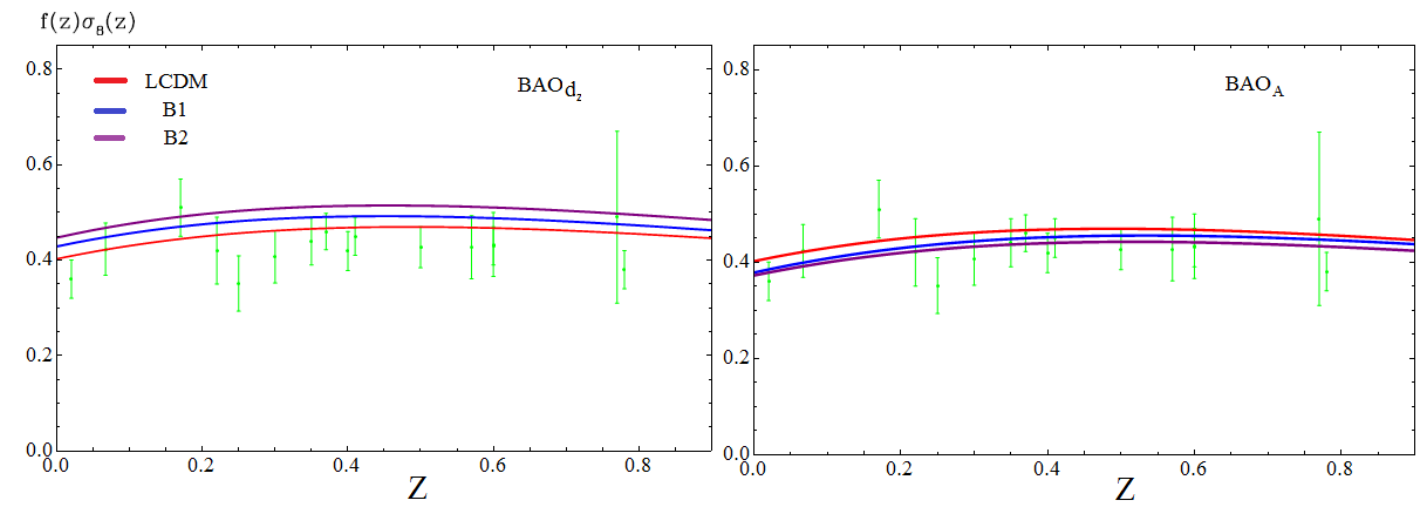

Figure 6: Comparison of the observed and theoretical evolution of the weighted growth rate $f(z) \sigma_{8}(z)$ for the models B1 and B2. As in Fig.5, the left and right panels show the results based on the $\mathrm{SNIa}+\mathrm{CMB}+\mathrm{BAO}_{d z}$ and $\mathrm{SNIa}+\mathrm{CMB}+\mathrm{BAO}_{A}$ best fit values, respectively. On the left panel, the lowermost (red) line corresponds to the $\Lambda$ CDM model, used as a reference. The closest one on top of it at $z=0$ corresponds to model B1 (blue line) and the next to closest at this point is model B2 (purple line). On the right panel, the $\mathrm{B} 1$ and $\mathrm{B} 2$ lines essentially overlap below the $\Lambda \mathrm{CDM}$ one, the $\mathrm{B} 1$ being in the middle.

to the variable $\ln a$. Therefore,

$$
f(a) \equiv \frac{1}{D} \frac{d D}{d \ln a}=\frac{d \ln D}{d \ln a}=-(1+z) \frac{d \ln D}{d z},
$$

where $D(a)$ is obtained from solving the differential equation (5.6) for each model. The physical significance of $f(a)$ is that it determines the amplitude of redshift distortions, and also of the peculiar velocity flows. The latter can be seen by witting $f(a)=(\dot{D} / D) /(\dot{a} / a)=$ $\dot{D} /(D H)$, which is the ratio of the peculiar flow rate to the Hubble rate.

In order to investigate the performance of our vacuum models, we compare the theoretical growth prediction with the latest growth data (as collected e.g. by [80] and references therein), which are based on the combined observable $f(z) \sigma_{8}(z)$, viz. the ordinary growth rate weighted by the rms mass fluctuation field. It has been found that this estimator is almost a model-independent way of expressing the observed growth history of the universe, in particular it is found to be independent of the galaxy density bias (see [81]). The theoretical functional form of $\sigma_{8}(z)$ will be studied in Sect.7.1 - see Eq.(7.8).

In Figs. 5 and 6 we display the predicted $f(z) \sigma_{8}(z)$ together with the observed linear growth data, for the various vacuum models $\mathrm{A}, \mathrm{B}$ and $\mathrm{C} 1$. No information is provided on $\mathrm{C} 2$ which we already discarded. Notice, that the theoretical curves on the left and the right panels correspond to fitted values of the cosmological parameters derived from $\mathrm{SNIa}+\mathrm{CMB}+\mathrm{BAO}_{d z}$ and $\mathrm{SNIa}+\mathrm{CMB}+\mathrm{BAO}_{A}$ respectively. Obviously, despite the fact that the $\mathrm{C} 1$ model fits well the expansion history (cf. Sect. 6.3), it is finally ruled out by the growth data [23]. For these reasons we come to the conclusion that the entire $\mathrm{C} 1$ class of models (in particular the linear sort $\Lambda \propto H$ ) is strongly unfavored and we do not 

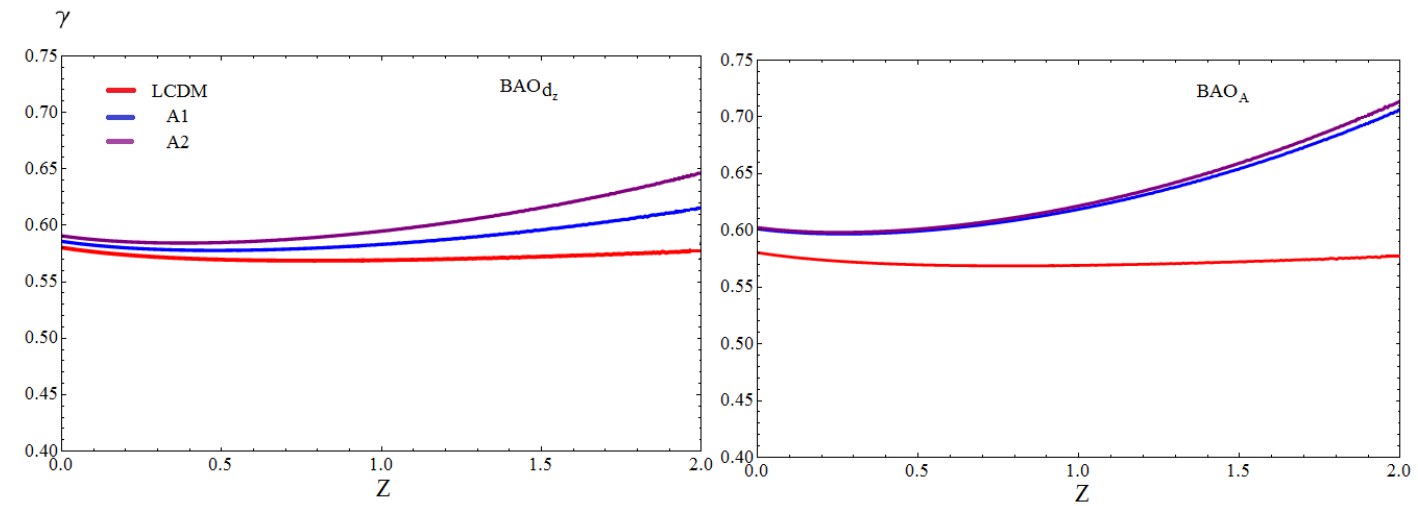

Figure 7: The evolution of the growth rate index, Eq. (6.26). The lines correspond to the A1 and A2 vacuum models for the best fit cosmological values discussed in Sec.6.3. From top to bottom (in both panels): A2 (purple line), A1 (blue line) and $\Lambda$ CDM (red line), used as reference. The left panel shows the results based on the $\mathrm{SNIa}+\mathrm{CMB}+\mathrm{BAO}_{d z}$ fitting while the right panel those of the $\mathrm{SNIa}+\mathrm{CMB}+\mathrm{BAO}_{A}$ analysis.

continue its analysis for the rest of this paper.

Overall, we can see that the A1-A2 vacuum models with the single parameter $\nu$ (or $\left.\nu_{\text {eff }}\right)$ match quite well the growth data, that is to say in a way which is comparable to the $\Lambda \mathrm{CDM}$ model (dashed line). We confirm this fact via a $\chi_{\text {growth }}^{2}$ minimization statistical test. In particular, for the vacuum models (including the $\Lambda \mathrm{CDM}$ ) we find that $\chi_{\text {growth }}^{2} / 16$ lies in the interval $[0.52-1.25]^{9}$.

On inspecting once more Figs. 5 and 6, the data clearly shows that the growth of structure is hindered near our time, which is evidence of a positive cosmological constant exerting a negative pressure against the process of matter collapse. This is well described by the $\Lambda$ CDM. But it is also comparably well described by the running vacuum models carrying an additive constant term in their functional form [see Eq. (2.8)] and a relatively small value of $\nu$ or $\epsilon$ of order $\sim 10^{-3}$. All these features can be seen very clearly in that figure.

Finally, let us finish with a short discussion concerning the growth rate index $\gamma$. As we have already mentioned in the introduction, we can express the linear growth rate of clustering in terms of $\Omega_{m}(z)$ as follows: $f(z) \simeq \Omega_{m}(z)^{\gamma(z)}$, where $\gamma$ is the linear growth rate index. For the usual $\Lambda$ CDM model, such index is approximated by $\gamma_{\Lambda} \simeq 6 / 11 \simeq 0.545$. This result is a particular case (for $\omega_{D}=-1$ ) of the theoretical formula $\gamma \simeq 3\left(\omega_{D}-1\right) /\left(6 \omega_{D}-5\right)$ corresponding to DE models with a slowly varying equation of state $\omega_{D}[82]$.

To obtain the linear growth index for the dynamical vacuum models studied here we have to use the corresponding linear growth factor, $D(z)$, and from Eq.(6.26) we easily

\footnotetext{
${ }^{9}$ The growth sample contains 16 entries [80].
} 
obtain:

$$
\gamma(z) \simeq \frac{\ln \left[-(1+z) \frac{d \ln D}{d z}\right]}{\ln \Omega_{m}(z)}
$$

where $D(z)$ for the different vacuum models is given in sections 5.2 and 5.3.

In Figs. 7 and 8 we present the evolution of the linear growth index for the $\mathrm{A}$ and $\mathrm{B}$ type of vacuum models, respectively (on the left with $\mathrm{SNIa}+\mathrm{CMB}+\mathrm{BAO}_{d z}$ data, and on the right with $\mathrm{SNIa}+\mathrm{CMB}+\mathrm{BAO}_{A}$ data). In the same figures we can also see our determination of $\gamma_{\Lambda}(z)$ as a function of the redshift, and in particular we find $\gamma_{\Lambda}(0) \simeq 0.58$.

The comparison shown in the mentioned figures indicates that the growth index of the type- $\mathrm{A}$ vacuum models with $\mathrm{SNIa}+\mathrm{CMB}+\mathrm{BAO}_{d z}$ data is well approximated by the $\Lambda \mathrm{CDM}$ constant value for $z \leq 1$, while at large redshifts there are deviations. When $\mathrm{SNIa}+\mathrm{CMB}+\mathrm{BAO}_{A}$ data is used, instead, there is a visible deviation from above in all the range, which becomes smaller (at the level $5 \%$ ) for $z \leq 1$ (see Fig. 7, right panel). Let us note that other vacuum models, such as e.g. B1 and B2, depart also from the $\Lambda$ CDM result (in this case from below) when using SNIa $+\mathrm{CMB}+\mathrm{BAO}_{d z}$ data (cf. Fig. 8, left panel). We find that for $z \leq 1$ the departure can be of order $5-10 \%$. The deviation, on the other hand, is not so pronounced (and of opposite sign) when $\mathrm{SNIa}+\mathrm{CMB}+\mathrm{BAO}_{A}$ data are used (right panel of the same figure).

It is worth mentioning that the differences we have found with respect to the $\Lambda \mathrm{CDM}$ are near the edge of the present experimental limits. For example, in a recent analysis of the clustering properties of Luminous Red Galaxies and the growth rate data provided by the various galaxy surveys it is found that $\gamma=0.56 \pm 0.05$ and $\Omega_{m}^{0}=0.29 \pm 0.01$ [39]. The prediction of $\gamma$ for all our vacuum models lies within $1 \sigma$ of that range.

Since the experimental error on the $\gamma$-index is of order $10 \%$ and some of the vacuum models are bordering these limits, it opens the possibility that the deviations presented by these models might be resolved in the future when more accurate data will be available [83]. This is quite evident from the results presented in Figs. 7 and 8 of our analysis. Combining the analysis of the growth rate with the those obtained from the cluster number counts method (studied in the next section), it should be possible to further pin down the nature of these dynamical vacuum models.

\section{Testing the dynamics of vacuum through the cluster number counts method}

In the foregoing part of our analysis we have shown that the A and B types of vacuum models can successfully fit the background cosmological data and the growth of linear perturbations in a way which in some cases is perfectly comparable to the $\Lambda$ CDM. This is not so with type-C models, which fail seriously in regard to the expansion data or the structure formation data or both. We have also shown that the A and B vacuum classes 

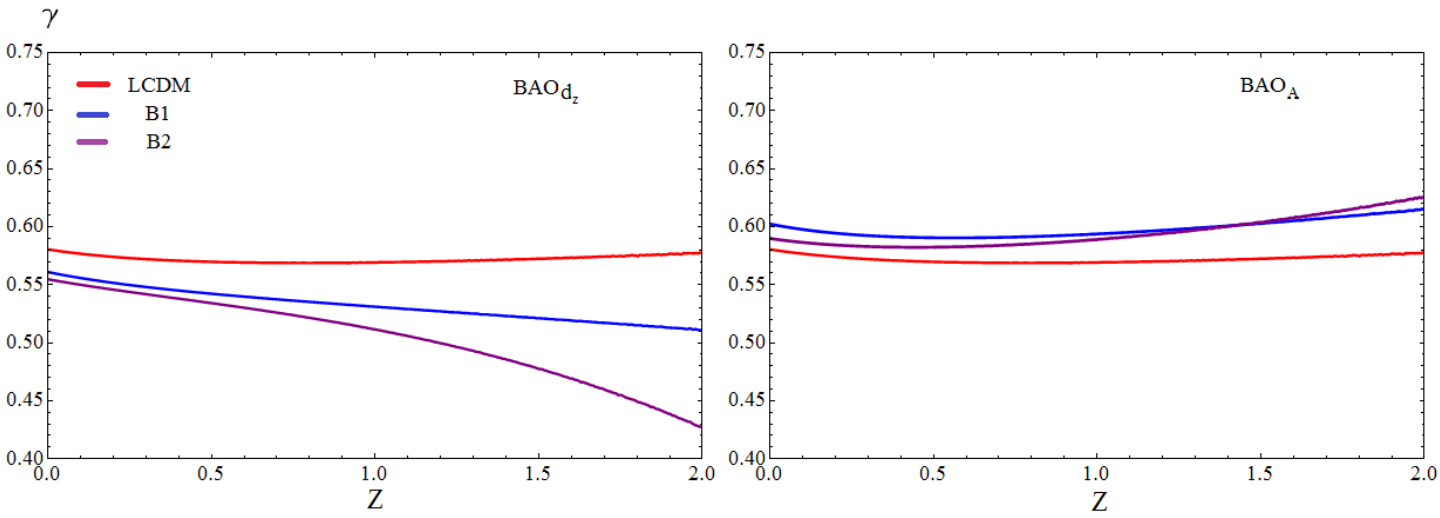

Figure 8: The evolution of the growth rate index, similar to the previous figure. In this case the lines correspond to the B1 and B2 vacuum models for the best fit cosmological values discussed in Sec. 6.3. The left panel shows the results based on the $\mathrm{SNIa}+\mathrm{CMB}+\mathrm{BAO}_{d z}$ fitting while the right panel those of the $\mathrm{SNIa}+\mathrm{CMB}+\mathrm{BAO}_{A}$ analysis. The uppermost curve (in red) on the left panel corresponds to the $\Lambda \mathrm{CDM}$, the middle one is for B1 (blue line) and the lowest one (in purple) is for B2. On the right panel the $\Lambda$ CDM curve is the lowest one. Near $z=0$ the highest one (in blue) is for B1 and the middle curve (in purple) is for B2.

\begin{tabular}{|c|c|cc|c|c|}
\hline \hline Model & $\Omega_{m}^{0}$ & $\nu$ & $\epsilon$ & $\sigma_{8}$ & $\delta_{c}$ \\
\hline$\Lambda \mathrm{CDM}$ & 0.292 & 0 & 0 & 0.829 & 1.675 \\
\hline $\mathrm{A} 1$ & 0.292 & +0.0013 & 0 & 0.813 & 1.666 \\
$\mathrm{~A} 2$ & 0.290 & +0.0024 & 0 & 0.797 & 1.659 \\
\hline $\mathrm{B} 1$ & 0.297 & 0 & -0.014 & 0.859 & 1.696 \\
$\mathrm{~B} 2$ & 0.300 & -0.0039 & -0.0039 & 0.896 & 1.705 \\
\hline
\end{tabular}

Table 3: Numerical results from fitting $\mathrm{SNIa}+\mathrm{CMB}+\mathrm{BAO}_{d z}$ data (in correspondence with Table 1 ). The $1^{\text {st }}$ column indicates the vacuum energy model. The $2^{\text {nd }}$ shows the central fit value of $\Omega_{m}^{0}$. The $3^{r d}$ and $4^{\text {th }}$ display the best fit values of the parameters $\nu$ and $\epsilon$, with the understanding that $\nu$ is to be taken $\nu_{\text {eff }}$ for model A2. Finally, the $5^{\text {th }}$ and $6^{\text {th }}$ columns list the computed values of $\sigma_{8}$ and $\delta_{c} \equiv \delta_{c}(z=0)$, respectively. The procedure to compute the collapse density threshold $\delta_{c}(z)$ for each model is explained in Appendix B.

have different predictions concerning the linear growth rate index $\gamma$, which in the future may be resolved. In that case we could distinguish between these two sort of vacuum models and also with respect to the $\Lambda$ CDM.

In the meanwhile and in an attempt to define further observational criteria capable of distinguishing the realistic model variants $\mathrm{A}$ and $\mathrm{B}$ from the concordance $\Lambda \mathrm{CDM}$ cosmology, we analyze in this second part of our work their theoretically predicted cluster-size halo redshift distributions, i.e. the expected cluster number counts of each model as a function of the redshift. As it turns, this is an efficient method to separate vacuum models which perform outstanding at the linear perturbation but differ very little in the values of the 
parameters.

In previous works some of us have described and tested this methodology for simpler versions of the dynamical vacuum models, see Refs. [19] and [20]. The method has also been used to place bounds on cosmological parameters and on different types of dark energy models, see e.g. [84, 85, 86]. The basic tool is the Press-Schechter formalism and its generalization. In the following we briefly summarize the basics of this method and refer the reader to the aforesaid references for more details. A crucial ingredient of the cluster number counts method is the linearly extrapolated density threshold above which structures collapse, $\delta_{c}$. The computation of this model-dependent parameter is a rather demanding task as it requires to solve the perturbations equations beyond the linear approximation. In the Appendix B we compute $\delta_{c}$ for the models under consideration.

\subsection{Generalized Press-Schechter formalism}

The Press and Schechter (hereafter PSc) formalism to compute the fraction of matter in the universe that has formed bounded structures and its redshift distribution was developed in a pioneering work of these authors 40 years ago [87] and has been generalized and improved since then. One introduces the so-called halo mass function, $F(M, z)$, representing the fraction of the universe that has collapsed by the redshift $z$ in halos above some mass $M$, where the primordial density fluctuation for a given mass $M$ of the dark matter fluid is described by a random Gaussian field. With this function and assuming a mean background mass density $\bar{\rho}$ one may estimate the (comoving) number density of virialized halos, $n(M, z)$, with masses within the range $(M, M+\delta M)$ :

$$
n(M, z) d M=\frac{\partial F(M, z)}{\partial M} \frac{\bar{\rho}}{M} d M .
$$

This expression can be rewritten as follows:

$$
n(M, z) d M=\frac{\bar{\rho}}{M} \frac{d \ln \sigma^{-1}}{d M} f_{\mathrm{PSc}}(\sigma) d M
$$

where $f_{\mathrm{PSc}}(\sigma)=\sqrt{2 / \pi}\left(\delta_{c} / \sigma\right) \exp \left(-\delta_{c}^{2} / 2 \sigma^{2}\right)$. Note that in this approach all the mass is locked inside halos, according to the normalization constraint:

$$
\int_{-\infty}^{+\infty} f_{\mathrm{PSc}}(\sigma) d \ln \sigma^{-1}=1
$$

The parameter $\delta_{c}$ is the collapse density threshold, i.e. the linearly extrapolated density threshold above which structures collapse [88] (see our Appendix B for more details), while $\sigma^{2}(M, z)$ is the mass variance of the smoothed linear density field, which depends on the redshift $z$ at which the halos are identified. It is given in Fourier space by:

$$
\sigma^{2}(M, z)=\frac{D^{2}(z)}{2 \pi^{2}} \int_{0}^{\infty} k^{2} P(k) W^{2}(k R) d k
$$




\begin{tabular}{|c|c|cc|c|c|}
\hline \hline Model & $\Omega_{m}^{0}$ & $\nu$ & $\epsilon$ & $\sigma_{8}$ & $\delta_{c}$ \\
\hline$\Lambda$ CDM & 0.292 & 0 & 0 & 0.829 & 1.675 \\
\hline $\mathrm{A} 1$ & 0.282 & +0.0048 & 0 & 0.758 & 1.644 \\
$\mathrm{~A} 2$ & 0.283 & +0.0048 & 0 & 0.757 & 1.642 \\
\hline $\mathrm{B} 1$ & 0.283 & 0 & +0.005 & 0.820 & 1.667 \\
$\mathrm{~B} 2$ & 0.283 & +0.0015 & +0.0015 & 0.791 & 1.662 \\
\hline
\end{tabular}

Table 4: As in Table 3, but using the fitting results from $\mathrm{SNIa}+\mathrm{CMB}+\mathrm{BAO}_{A}$ data (in correspondence with Table 2).

In this expression, $D(z)$ is the linear growth factor of perturbations, which we have computed before for our models, $P(k)$ is the power-spectrum of the linear density field, and finally we have the smoothing function $W(k R)=3(\sin k R-k R \cos k R) /(k R)^{3}$, which is the Fourier image of the following geometric top-hat function with spherical symmetry: $f_{\mathrm{TH}}(r)=3 /\left(4 \pi R^{3}\right) \theta(1-r / R)$. Here $\theta(1-r / R)$ is the Heaviside function, which implements the top-hat spherical truncation for $r>R$. Notice that, thanks to it, its Fourier transform also truncates the sum over modes in (7.4) since $W(k R) \simeq 1$ for $k R \ll 1$, but $W(k R) \simeq 0$ for $k R \gg 1$. The spherical distribution contains on average a mass $M$ within a radius $R=(3 M / 4 \pi \bar{\rho})^{1 / 3}$. In this expression $\bar{\rho}$ is the comoving mean mass density at redshift $z$, i.e. the mean mass density of the background divided by $(1+z)^{3}$. The current background density is $\rho_{m}=\Omega_{m}^{0} \rho_{c}^{0}=2.78 \times 10^{11} \Omega_{m}^{0} h^{2} M_{\odot} \mathrm{Mpc}^{-3}$. We use the CDM power spectrum:

$$
P(k)=P_{0} k^{n_{s}} T^{2}\left(\Omega_{m}^{0}, k\right),
$$

where $P_{0}$ is a normalization cconstant (see below), $n_{s}=0.9603 \pm 0.0073$ is the value of the spectral index measured by Planck+WP $[1]$; and $T\left(\Omega_{m}^{0}, k\right)$ the BBKS transfer function $[89,90]$ :

$$
\begin{aligned}
& T\left(\Omega_{m}^{0}, k\right)=\frac{\ln (1+2.34 q)}{2.34 q}\left[1+3.89 q+(16.1 q)^{2}+(5.46 q)^{3}+(6.71 q)^{4}\right]^{-1 / 4} \\
& q=q(k) \equiv \frac{k \cdot \mathrm{Mpc}}{\Omega_{m}^{0} h^{2}} e^{\Omega_{b}^{0}+\sqrt{2 h} \frac{\Omega_{b}^{0}}{\Omega_{m}^{0}}}
\end{aligned}
$$

It is traditional to parameterize the mass variance in terms of $\sigma_{8}$, the rms mass fluctuation amplitude on scales of $R_{8}=8 h^{-1} \mathrm{Mpc}$ at redshift $z=0\left[\sigma_{8} \equiv \sigma_{8}(0)\right]$. This allows us to normalize the power spectrum, i.e. to determine $P_{0}$. Indeed, using equations (7.4) and (7.5) we have:

$$
\sigma^{2}(M, z)=\sigma_{8}^{2}(z) \frac{\int_{0}^{\infty} k^{n_{s}+2} T^{2}\left(\Omega_{m}^{0}, k\right) W^{2}(k R) d k}{\int_{0}^{\infty} k^{n_{s}+2} T^{2}\left(\Omega_{m}^{0}, k\right) W^{2}\left(k R_{8}\right) d k},
$$

where

$$
\sigma_{8}(z)=\sigma_{8} \frac{D(z)}{D(0)}
$$




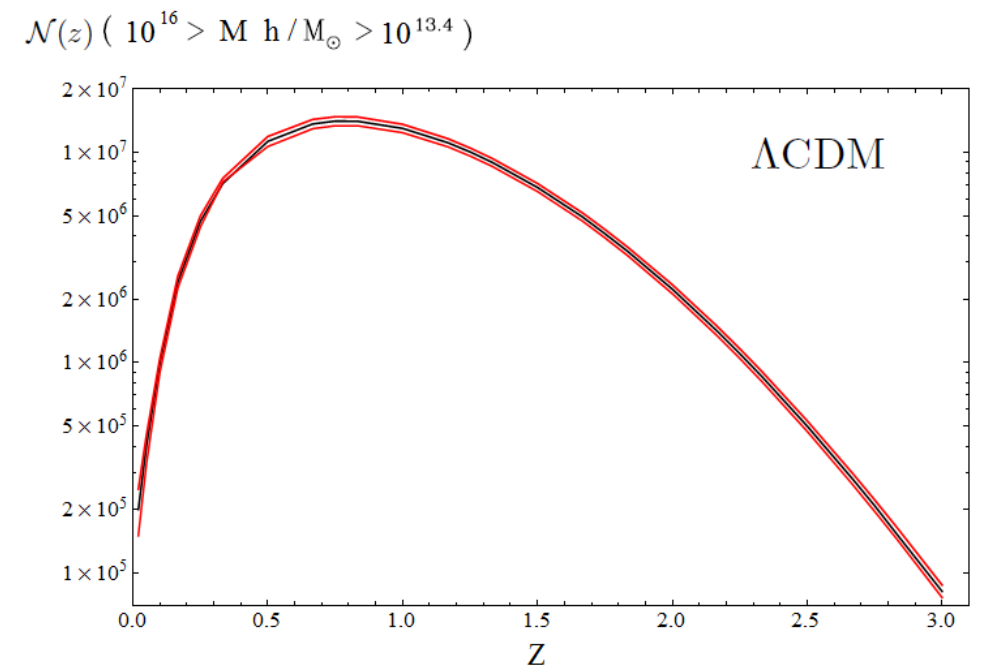

Figure 9: The theoretically predicted redshift distribution of the total number of cluster counts, $\mathcal{N}(z)$, with masses in the range $10^{13.4} h^{-1} \lesssim M / M_{\odot} \lesssim 10^{16} h^{-1}$, corresponding to the concordance $\Lambda$ CDM model and using the generalized Press-Schechter function (7.12). The curves correspond to the best fit value $\Omega_{m}^{0}=0.293 \pm 0.013$ within $1 \sigma$ (cf. Table 1$)$.

Equivalently,

$$
P_{0}=2 \pi^{2} \frac{\sigma_{8}^{2}}{D^{2}(0)}\left[\int_{0}^{\infty} k^{n_{s}+2} T^{2}\left(\Omega_{m}^{0}, k\right) W^{2}\left(k R_{8}\right) d k\right]^{-1} .
$$

The Planck+WP value of $\sigma_{8}$, which we use for our analysis, is $\sigma_{8}=0.829 \pm 0.012[1]$.

The $\sigma_{8}$ value for the different dynamical vacuum models can be estimated by scaling the present time $\Lambda \mathrm{CDM}$ value $^{10}\left(\sigma_{8, \Lambda}\right)$ using once more equations (7.4) and (7.5):

$$
\sigma_{8}=\sigma_{8, \Lambda} \frac{D(0)}{D_{\Lambda}(0)}\left[\frac{\int_{0}^{\infty} k^{n_{s}+2} T^{2}\left(\Omega_{m}^{0}, k\right) W^{2}\left(k R_{8}\right) d k}{\int_{0}^{\infty} k^{n_{s}+2} T^{2}\left(\Omega_{m, \Lambda}^{0}, k\right) W^{2}\left(k R_{8}\right) d k}\right]^{1 / 2} .
$$

Overall it follows from the foregoing formulae that the mass variance of the linear density field is determined from

$$
\sigma^{2}(M, z)=\sigma_{8, \Lambda}^{2} \frac{D^{2}(z)}{D_{\Lambda}^{2}(0)} \frac{\int_{0}^{\infty} k^{n_{s}+2} T^{2}\left(\Omega_{m}^{0}, k\right) W^{2}(k R) d k}{\int_{0}^{\infty} k^{n_{s}+2} T^{2}\left(\Omega_{m, \Lambda}^{0}, k\right) W^{2}\left(k R_{8}\right) d k},
$$

where $\sigma_{8, \Lambda} \simeq 0.829$ is the aforementioned $\Lambda \mathrm{CDM}$ value extracted from Planck+WP measurements. Furthermore, the numerical value of $\sigma_{8}$ for the $\Lambda \mathrm{CDM}$ and the various vacuum models under consideration has been collected in the last but one column of Tables 3 and 4 together with the best fitting values of the parameters according to each BAO type that we have used (cf. Tables 1 and 2).

\footnotetext{
${ }^{10}$ In the following discussion, the quantities referred to the $\Lambda$ CDM model are distinguished by the subscript ' $\Lambda$ ' $\left(\sigma_{8, \Lambda} ; D_{\Lambda} ; \Omega_{m, \Lambda}^{0}\right)$ whereas the corresponding quantities in the dynamical vacuum models carry no subscript.
} 

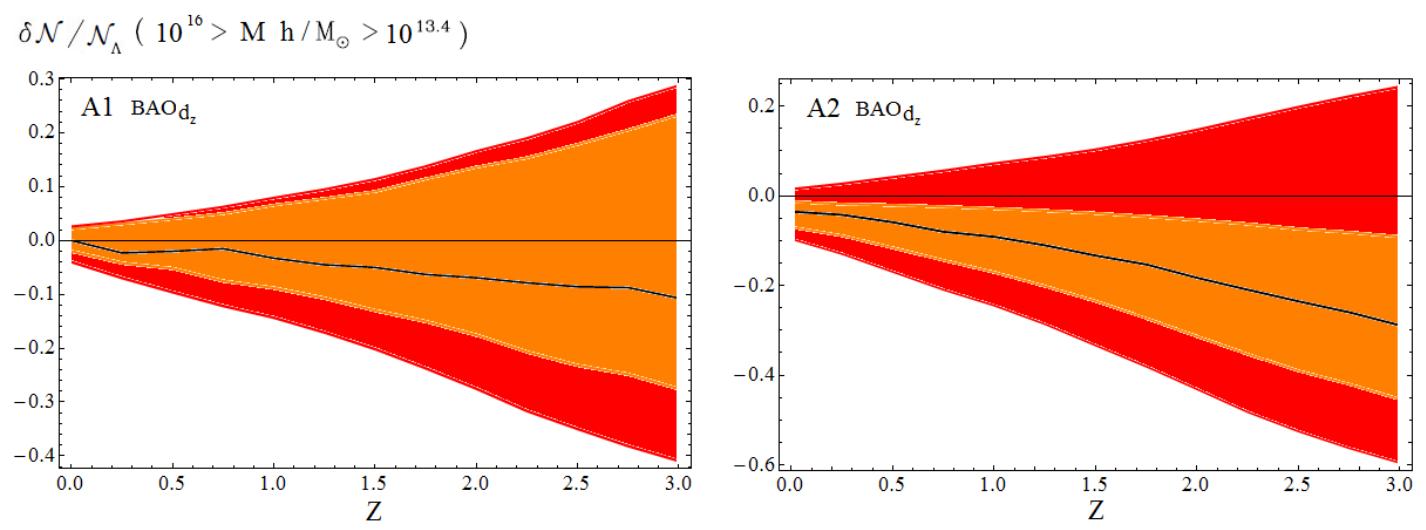

Figure 10: Left: Fractional difference $\delta \mathcal{N} / \mathcal{N}$ in the number of counts of clusters between the vacuum model A1 and the concordance $\Lambda$ CDM model (cf. Fig. 9) using SNIa+CMB+BAO $d z$ data from Table 1. The continuous solid line represents $\delta \mathcal{N} / \mathcal{N}$ for the best fit value from that table, whereas the innermost (resp. outermost) band comprises the $\delta \mathcal{N} / \mathcal{N}$ prediction for the points within the $\pm 1 \sigma$ (resp. $\pm 3 \sigma$ ) values around it. Right: As before, but for model A2.

The original Press-Schechter function $f_{\mathrm{PSc}}$ was shown to provide a relatively good first approximation to the halo mass function obtained by numerical simulations. In Appendix A we use $f_{\mathrm{PSc}}$ to assess in detail why the number count method is an efficient one to separate models that may be difficult to distinguish at the linear perturbation regime. The method, however, is not tied to the particularly simple form of the original Press-Schechter function $f_{\mathrm{PSc}}$. More recently a large number of works have provided better fitting functions for $f(\sigma)$. In practice, in our analysis for the various dynamical vacuum models under consideration we will adopt the generalized one proposed by Reed et al. [91]:

$$
\begin{aligned}
f_{R}\left(\sigma, n_{\mathrm{eff}}\right)=A \sqrt{\frac{2 b}{\pi}} & {\left[1+\left(\frac{\sigma^{2}}{b \delta_{c}^{2}}\right)^{p}+0.6 G_{1}+0.4 G_{2}\right]\left(\frac{\delta_{c}}{\sigma}\right) } \\
& \times \exp \left[-\frac{c b \delta_{c}^{2}}{2 \sigma^{2}}-\frac{0.03}{\left(n_{\mathrm{eff}}+3\right)^{2}}\left(\frac{\delta_{c}}{\sigma}\right)^{0.6}\right],
\end{aligned}
$$

where $A=0.3222, p=0.3, b=0.707, c=1.08$, while $G_{1}, G_{2}$ and $n_{\text {eff }}$, the slope of the non-linear power-spectrum at the halo scale, are given by:

$$
G_{1}=\exp \left[-\frac{\left(\ln \sigma^{-1}-0.4\right)^{2}}{2(0.6)^{2}}\right], G_{2}=\exp \left[-\frac{\left(\ln \sigma^{-1}-0.75\right)^{2}}{2(0.2)^{2}}\right], n_{\mathrm{eff}}=6 \frac{\mathrm{d} \ln \sigma^{-1}}{\mathrm{~d} \ln M}-3
$$

The previous generalized PS function is a refined variant of an older function that was used to improve the original PS-formalism by Sheth and Tormen [92]:

$$
f_{S T}(\sigma)=A^{\prime} \sqrt{\frac{2 b}{\pi}}\left[1+\left(\frac{\sigma^{2}}{b \delta_{c}^{2}}\right)^{p}\right]\left(\frac{\delta_{c}}{\sigma}\right) \exp \left[-\frac{b \delta_{c}^{2}}{2 \sigma^{2}}\right]
$$



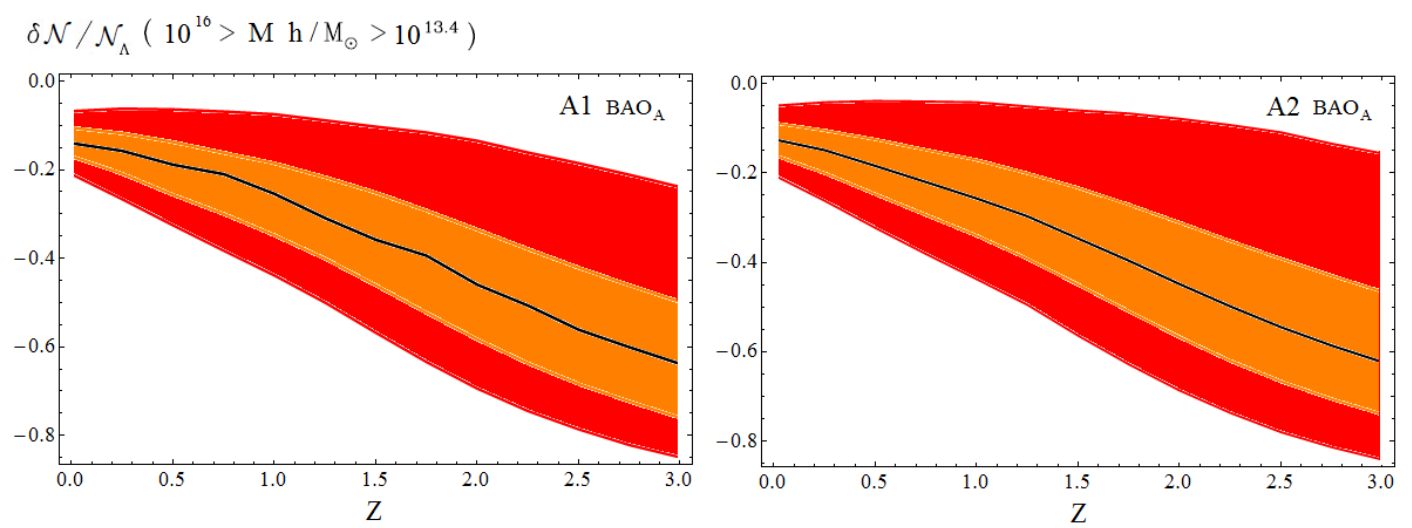

Figure 11: Fractional difference $\delta \mathcal{N} / \mathcal{N}$ in the number of counts of clusters between the vacuum models $\mathrm{A} 1$ (left) and A2 (right) and the concordance $\Lambda \mathrm{CDM}$ model, but using $\mathrm{SNIa}+\mathrm{CMB}+\mathrm{BAO}_{A}$ data from Table 2. Same notation as in Fig. 10.

where the parameters $b$ and $p$ are the same as in (7.12). Once more $A^{\prime}$ must be fixed from the normalization (7.3). Let us, however, note that the value of the normalization constant cancels in the ratio $\delta \mathcal{N} / \mathcal{N}_{\Lambda \mathrm{CDM}}$, where $\delta \mathcal{N}=\mathcal{N}-\mathcal{N}_{\Lambda \mathrm{CDM}}$ represents the deviations of the number counts of the given vacuum model with respect to the $\Lambda$ CDM. In fact, the fractional difference $\delta \mathcal{N} / \mathcal{N}_{\Lambda \mathrm{CDM}}$ will be the main observable in our test analysis of the number counts for dynamical vacuum models. While we have also made use of the parameterization (7.14) to test the sensibility of our results to the generalized Press-Schechter functions, we will for definiteness only present the final results in terms of the more complete function (7.12).

To use that function we need to know the value of the collapse density threshold parameter $\delta_{c}$. In Appendix B we compute $\delta_{c}$ by solving the corresponding nonlinear perturbation equations for each vacuum model. The resulting values are listed in the last column of Tables 3 and 4, where we have separated them according to the type of BAO used in the best fitting to the $\mathrm{SNIa}+\mathrm{CMB}+\mathrm{BAO}$ cosmological data.

We conclude this section by noticing that the BBKS transfer function (7.6), as well as the PS-like functions (7.12) and (7.14) involved in the halo mass function, were all obtained from fits to numerical data assuming strict $\Lambda \mathrm{CDM}$ cosmology [89, 91, 92]. We have checked e.g. that the differences in number counts between the mentioned mass functions are within the errors induced in the determination of the model parameters. In this sense we do not consider necessary at this point to further adapt the model dependence of these functions beyond our detailed computation of the $\delta_{c}$ parameter for each model (cf. Appendix B). The results of our number count analysis (see the next section) within these approximations are already quite suggestive of the rich spectrum of possibilities offered by the dynamical models under study. However, we understand that with the advent of more precision data in the future a more refined treatment might be necessary. 

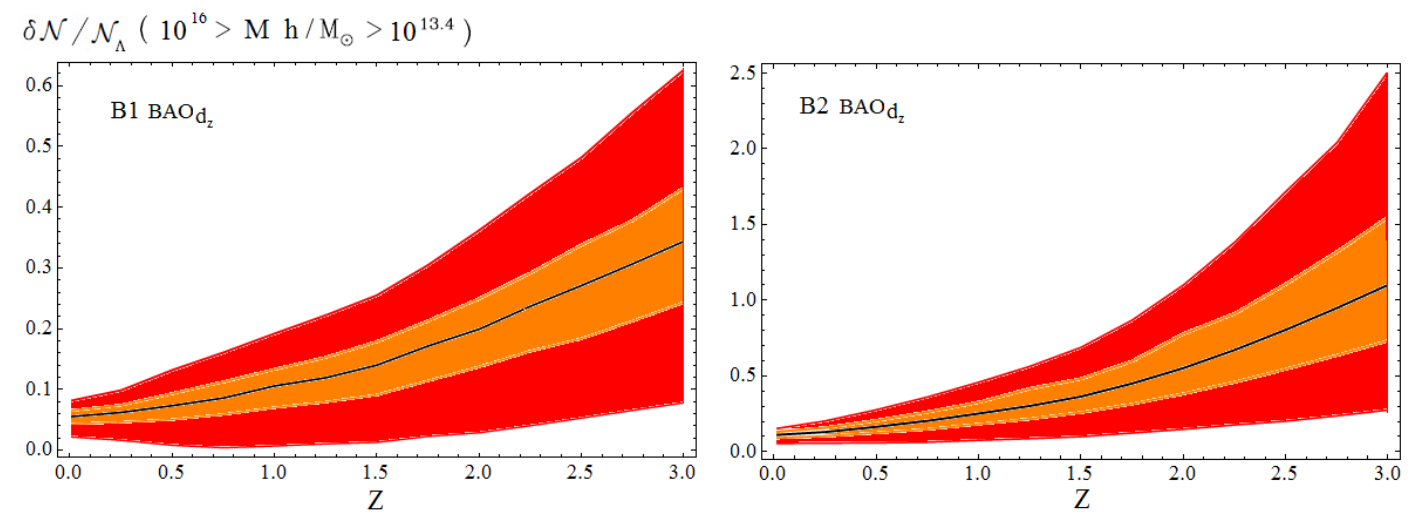

Figure 12: Fractional difference $\delta \mathcal{N} / \mathcal{N}$ in the number of counts of clusters between the vacuum models $\mathrm{B} 1$ (left) and B2 (right) and the concordance $\Lambda \mathrm{CDM}$ model, using SNIa $+\mathrm{CMB}+\mathrm{BAO}_{d z}$ data from Table 1. Same notation as in Fig. 10.

\subsection{Numerical results: number counts of the dynamical vacuum models}

From the halo mass function (7.2) we can derive for each vacuum model the redshift distribution of clusters, $\mathcal{N}(z)$, within some determined mass range, say $M_{1} \leq M \leq M_{2}$. This can be estimated by integrating the expected differential halo mass function, $n(M, z)$, with respect to mass, namely

$$
\mathcal{N}(z)=\frac{d V}{d z} \int_{M_{1}}^{M_{2}} n(M, z) d M
$$

where $d V / d z$ is the comoving volume element, which in a flat universe takes the form:

$$
\frac{d V}{d z}=4 \pi r^{2}(z) \frac{d r(z)}{d z}
$$

with $r(z)$ denoting the comoving radial distance out to redshift $z$ :

$$
r(z)=\frac{c}{H_{0}} \int_{0}^{z} \frac{d z^{\prime}}{E\left(z^{\prime}\right)} .
$$

It follows that

$$
\mathcal{N}(z)=4 \pi r^{2}(z) \frac{d r}{d z} \int_{M_{1}}^{M_{2}} n(M, z) d M=-\frac{4 \pi r^{2} \bar{\rho}(z)}{H_{0} E(z)} \int_{M_{1}}^{M_{2}} \frac{1}{M}\left(\frac{1}{\sigma} \frac{d \sigma}{d M}\right) f(\sigma) d M .
$$

In practice, as we have said, we will use the function (7.12) for $f(\sigma)$ in the above expression.

In Fig. 9, we show the theoretically predicted redshift distribution of the total number of cluster counts, $\mathcal{N}(z)$, with masses in the range $10^{13.4} h^{-1} \lesssim M / M_{\odot} \lesssim 10^{16} h^{-1}$, corresponding to the concordance $\Lambda \mathrm{CDM}$ model. Notice that there is no significant difference in the best fitted $\Lambda \mathrm{CDM}$ value of $\Omega_{m}^{0}$ when we employ $\mathrm{SNIa}+\mathrm{CMB}+\mathrm{BAO}_{d z}$ or $\mathrm{SNIa}+\mathrm{CMB}+\mathrm{BAO}_{A}$ data, as can be seen in Tables 1 and 2 , and therefore the number of 

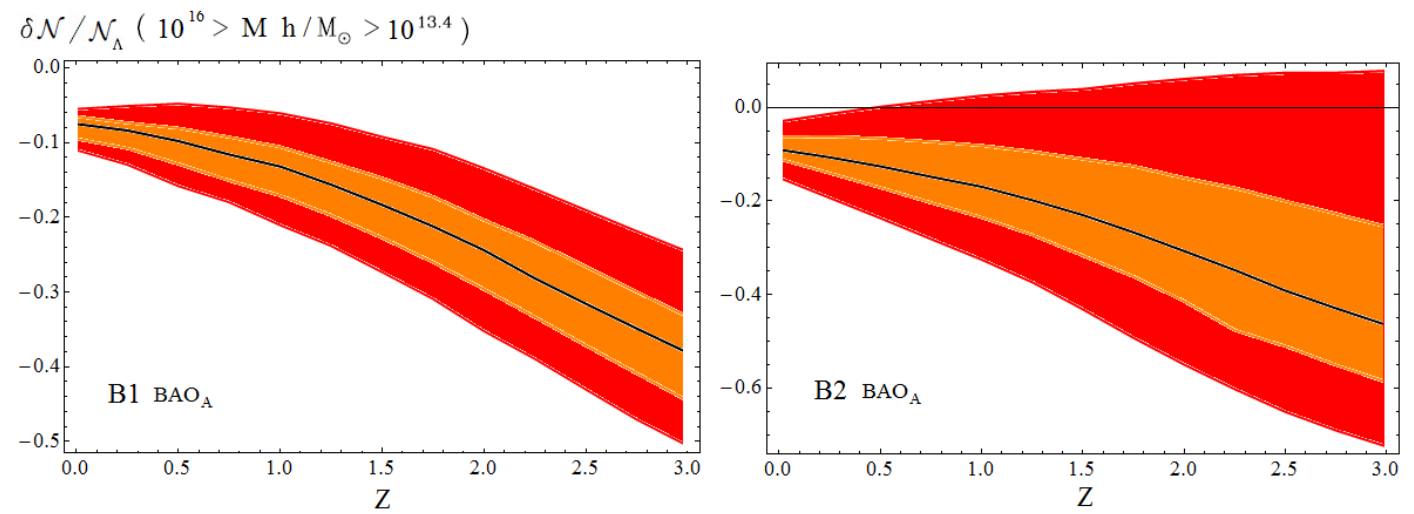

Figure 13: Fractional difference $\delta \mathcal{N} / \mathcal{N}$ in the number of counts of clusters between the vacuum models $\mathrm{B} 1$ (left) and B2 (right) and the concordance $\Lambda \mathrm{CDM}$ model, using $\mathrm{SNIa}+\mathrm{CMB}+\mathrm{BAO} A$ data from Table 2. Same notation as in Fig. 10.

counts in Fig. 9 does not depend on the BAO data used in the fit. We can see that the total number of counts increases with the redshift up to a maximum point and then decreases steadily, meaning that from that point onwards the larger is the redshift the smaller is the number of counts of virialized halos with a mass $M$ in the indicated range. The curves shown in that figure (which include the $1 \sigma$ error in the fitted value of $\Omega_{m}^{0}$ ) define the fiducial $\Lambda \mathrm{CDM}$ prediction. We will use it to compare with the corresponding outcome from the dynamical vacuum models under study. Recall that we denote the deviations of the number counts of a given vacuum model with respect to the $\Lambda \mathrm{CDM}$ as $\delta \mathcal{N}=\mathcal{N}-\mathcal{N}_{\Lambda \mathrm{CDM}}$.

We start our comparison by considering Fig. 10, where we display (on the left plot of it) the fractional difference $\delta \mathcal{N} / \mathcal{N}$ in the number of counts of clusters between the vacuum model A1 and the concordance $\Lambda \mathrm{CDM}$ model (cf. Fig. 9) using $\mathrm{SNIa}+\mathrm{CMB}+\mathrm{BAO} d z$ fitting data from Table 1. The continuous solid line in the figure represents the predicted deviation $\delta \mathcal{N} / \mathcal{N}$ for the best fit value from that table, whereas the inner and outer bands comprise the $\delta \mathcal{N} / \mathcal{N}$ prediction for the points within $\pm 1 \sigma$ and $\pm 3 \sigma$ values around it, respectively. The plot on the right of Fig. 10 is similar, but for model A2. The corresponding results for models $\mathrm{A} 1$ and $\mathrm{A} 2$ when $\mathrm{SNIa}+\mathrm{CMB}+\mathrm{BAO}_{A}$ fitting data from Table 2 are used can be seen in the two plots of Fig. 11.

We can summarize the analysis presented in Figs. 10 and 11 by saying that $\delta \mathcal{N} / \mathcal{N}$ can have both signs in the case of using $\mathrm{BAO}_{d z}$ data, provided we consider the points in the $\pm 3 \sigma$ band. The narrower $\pm 1 \sigma$ band is nevertheless more predominantly bent into the negative sign. As for the $\mathrm{BAO}_{A}$ data, the prediction for $\delta \mathcal{N} / \mathcal{N}$ is negative for all points, even for those in the $\pm 1 \sigma$ band. It means that, all in all, models A1 and A2 tend to predict a smaller number of counts as compared to the $\Lambda \mathrm{CDM}$. The fractional decrease can be as significant as $30-60 \%$.

The corresponding deviations in the number of counts for models B1 and B2 are 
depicted in Figs. 12 and 13. Here we find a feature that was not present for type-A models, namely we observe from these figures that the deviations with respect to the $\Lambda \mathrm{CDM}$ are all positive when the $\mathrm{BAO}_{d z}$ data are used (cf. Fig. 12), whilst they are negative when the $\mathrm{BAO}_{A}$ data are utilized (cf. Fig. 13). This may seem surprising, but is related to the sensitivity of the number counts to the best fit value of $\Omega_{m}^{0}$ employed in the analysis, which is different for each type of BAO. As we have seen from Table 1, the $\mathrm{BAO}_{d z}$ fitting data projects a value of $\Omega_{m}^{0}$ that is closer to the $\Lambda \mathrm{CDM}$ value than in the case of $\mathrm{BAO}_{A}$ (cf. Table 2). In the latter, $\Omega_{m}^{0}$ is significantly smaller than in the $\Lambda$ CDM model. The sign of $\delta \mathcal{N} / \mathcal{N}$ is tied to this fact. As it is shown in Appendix A, if a given vacuum model has the same $\Omega_{m}^{0}$ value (or very similar), the sign of $\delta \mathcal{N} / \mathcal{N}$ is opposite to the sign of the vacuum parameter $\nu$ or $\epsilon$ that dominates the model. For models $\mathrm{B} 1$ and $\mathrm{B} 2$ with $\mathrm{BAO}_{d z}$ data, the best fit value of $\Omega_{m}^{0}$ is indeed very close to the fitted value for the $\Lambda$ CDM. Thus, since for these models $\epsilon<0$ we find $\delta \mathcal{N} / \mathcal{N}>0$ and moreover this fraction is growing quite fast, up to $50-100 \%$ and more (Fig. 12). At variance with this situation, with $\mathrm{BAO}_{A}$ data these models predict a substantial depletion in the number counts as compared to the $\Lambda \mathrm{CDM}$, as shown in Fig. 13, the reason being the smaller preferred value of $\Omega_{m}^{0}$ as compared to the concordance model.

In Fig. 14 we have put in a nutshell the essential results of our number counts analysis. Namely, we have displayed the fractional differences $\delta \mathcal{N} / \mathcal{N}$ with respect to the $\Lambda \mathrm{CDM}$ by using only the best fit values of all the vacuum models in the two BAO modalities. Obviously we need an improvement of the two sorts of BAO measurements to see it they can eventually provide a more coincident best fit value of $\Omega_{m}^{0}$, as this is essential to decide on the sign of $\delta \mathcal{N} / \mathcal{N}$. From our point of view perhaps the least model-dependent BAO results are those from $\mathrm{BAO}_{A}$, as they are based on low-z data only and therefore are not so tied to the specific behavior of the models around the drag epoch.

Finally, as a particular case of our general treatment of type-A and type-B models, we briefly mention the situation with the class of $\mathrm{C} 1$ models (where e.g. the linear model $\rho_{\Lambda} \propto H$ is also included). We have emphasized in Sect.6.5 that the $\mathrm{C} 1$ models perform a rather bad fit to the linear growth of density perturbations. Recently, however, the number count analysis of the linear model $\rho_{\Lambda} \propto H$ has been considered in Ref. [52], in which a significant excess in the number of counts is reported as compared to the $\Lambda \mathrm{CDM}$ model. Although it is not part of our main purpose, we have computed in passing the corresponding number of counts for this model. Unfortunately, we do not concur with the results of [52]. We do not find an excess in the number of counts as compared to the $\Lambda \mathrm{CDM}$, but a large deficit.

To conclude, in view of the results found in our analysis of the cluster halo redshift distribution presented in Figures 10-14, we can assert that it is an efficient method to distinguish the various sorts of dynamical vacuum models with respect to the $\Lambda \mathrm{CDM}$ and also among themselves, especially when the different sources of BAO data will become 

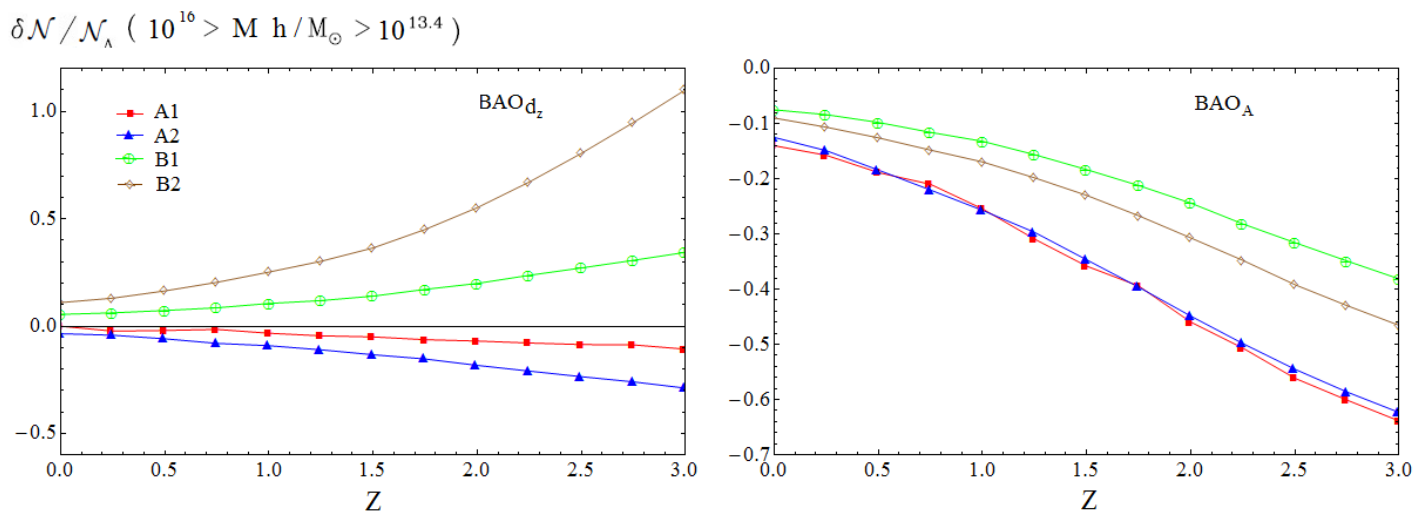

Figure 14: Left: Comparison of the fractional difference $\delta \mathcal{N} / \mathcal{N}$ in the redshift distribution of cluster number counts of all the vacuum models with respect to the concordance $\Lambda$ CDM model using the central fit values of the $\mathrm{SNIa}+\mathrm{CMB}+\mathrm{BAO}_{d z}$ data (cf. Table 1); Right: As before, but for the $\mathrm{SNIa}+\mathrm{CMB}+\mathrm{BAO}_{A}$ data (cf. Table 2).

more precise. The sensitivity of the method to the parameters $(\nu, \alpha, \epsilon, \ldots)$ of the vacuum models is large if we take into account that they are relatively small. We have found that the fractional differences $\delta \mathcal{N} / \mathcal{N}$ with respect to the $\Lambda \mathrm{CDM}$ can typically be as large as $\pm 50 \%$ despite the fact that the (absolute) values of those parameters are typically of order $10^{-3}$.

\section{Discussion and conclusions}

In this paper, we have analyzed in great detail several classes of dynamical vacuum models in which the vacuum energy density can be expressed as a power series of the Hubble function and its cosmic time derivative. We have singled out model types which are particularly attractive from the theoretical point of view, namely vacuum models for which the number of time derivatives of the scale factor is even: $\rho_{\Lambda}(t)=c_{0}+\sum_{k=1} \alpha_{k} H^{2 k}(t)+\sum_{k=1} \beta_{k} \dot{H}^{k}(t)$. These can be well motivated within the context of quantum field theory (QFT) in curved spacetime since their structure is manifestly compatible with the general covariance of the effective action and can be linked to the notion of renormalization group. For the study of the current universe the series naturally terminates at the level of the $H^{2}$ and $\dot{H}$ terms, but the higher order ones can be very important for a proper description of the early universe and the inflationary phase.

We have stressed the need for the nonvanishing additive (constant) term, $c_{0} \neq 0$, in the above class of models. It guarantees a smooth limit converging to the standard $\Lambda \mathrm{CDM}$ model when the coefficients of the dynamical terms go to zero. We have verified that models with $c_{0}=0$ are generally in conflict with observations, whether with the background data, or with the structure formation data, or both. 
For instance, we have considered vacuum models of the form $\Lambda=a_{0}+a_{1} \dot{H}+a_{2} H^{2}$, with $c_{0} \neq 0$ (the class of models that we have called type-A). They are well-behaved and if the (dimensionless) coefficients $a_{1}$ and $a_{2}$ are sufficiently small, the cosmological term develops just a mild dynamical behavior around the $\Lambda$ CDM model. Such framework could compete as a good candidate for a consistent description of the Universe in terms of dynamical vacuum energy, an option that should be considered natural in QFT in curved space-time.

In our analysis we have also admitted the possibility that some terms in the effective structure of $\Lambda(H, \dot{H})$ could mildly violate the covariance requirement on phenomenological grounds. Notwithstanding, we considered this possibility viable only when the expected terms are also present. We do not deem theoretically sound those vacuum models exclusively constructed from noncanonical terms (i.e. unexpected terms not satisfying the above mentioned conditions), such as e.g. the model $\Lambda \propto H$. A model of this sort has the double inconvenience that $c_{0}=0$ and that the number of time derivatives of the scale factor is odd (one derivative in this case). Not surprisingly when such model is confronted with observations fails on several accounts. When we add up to it the power $H^{2}$, we reach the model $\Lambda=c_{1} H+c_{2} H^{2}$ (referred to in this work as the type-C1 model). In this extended form the situation of the new model improves at the background level, but is still troublesome at the perturbations level since the model fails to describe the linear growth of structure formation. Similarly, the pure quadratic model $\Lambda \propto H^{2}$ is problematic, but for a different reason. While this model contains an even power of the Hubble rate, it is actually not sufficient to comply with the phenomenological requirement in the absence of a constant additive term. The reason is that it does not admit an inflection point from deceleration to acceleration, and moreover it does not have a growing mode for structure formation (for reasonable values of the cosmological parameters). The pure linear and quadratic models, $\Lambda \propto H$ and $\Lambda \propto H^{2}$, are therefore strongly excluded; and their combination, $\Lambda=c_{1} H+c_{2} H^{2}$, provides a model still considerably crippled to account for the structure formation data. Similar (though not identical) criticisms can be applied to vacuum models of the sort $\Lambda=c_{1} \dot{H}+c_{2} H^{2}$ (type-C2). Hence, all type-C models are unfavored, strongly disfavored or simply ruled out.

We have already mentioned that type-A dynamical vacuum models are in very good shape inasmuch as they are perfectly comparable to the concordance $\Lambda$ CDM model when the dynamical components are subdominant in the current universe. Interestingly, another viable variant that we have considered are the type-B models. These are obtained by including an additive term to the type-C1 models, i.e. they have the structure $\Lambda=b_{0}+$ $b_{1} H+b_{2} H^{2}$ with $b_{0} \neq 0$. We have found that they are also, in principle, phenomenologically admissible. For them the presence of the linear component is not so determinant as in the case of the type-C1, because it can be interpreted as a correction (e.g. a bulk viscosity effect) to the main structure. Most important, the type-B models have a smooth $\Lambda \mathrm{CDM}$ limit as in the type-A case, and this fact is again crucial to protect them from departing 
exceedingly from the concordance $\Lambda$ CDM model near our time.

In the present work we have solved the background and perturbations cosmology for all these vacuum models and confronted them with observations. In the light of the most recent observational data on type Ia supernovae, the Cosmic Microwave Background and the Baryonic Acoustic Oscillations (BAO), we have obtained a fit to their basic parameters $(\nu, \alpha, \epsilon)$. From the fitted values we have computed the linear growth factor of structure formation for each model and compared with the observed linear growth rate of clustering measured from the SDSS galaxies. Subsequently we have moved to the nonlinear regime and considered the predicted redshift distribution of cluster-size collapsed structures as a powerful method to distinguish the models. We have computed the corresponding fractional deviation $\delta \mathcal{N} / \mathcal{N}$ in the number of counts of clusters with respect to the $\Lambda \mathrm{CDM}$ prediction.

The general conclusion we have reached is that the studied dynamical vacuum models (type-A and type-B with nonvanishing additive constant term) are able to pass (with some differences) the combined observational tests, including the structure formation data, with a statistical significance that in some cases is comparable or even better than that of the concordance $\Lambda \mathrm{CDM}$ model. The current Universe appears in all these models as FLRWlike, except that the vacuum energy is not a rigid quantity but a mildly evolving one. In fact, the typical values we have obtained for the coefficients $\nu, \alpha$ and $\epsilon$ responsible for the time evolution of $\rho_{\Lambda}$ in these models lie in the ballpark of $\sim 10^{-3}$. This order of magnitude value is roughly consistent with the theoretical expectations, some of them interpreted in QFT as one-loop $\beta$-functions of the running cosmological constant.

Despite the two types of viable dynamical vacuum models remain close to the $\Lambda \mathrm{CDM}$ model, the overall fit from type-B models is not so good as the type-A ones. We have pointed out that this may be due to the fact that the presence of the linear term $\sim \epsilon H$ (characteristic of type-B models, especially the type-B1 ones) is unexpected in the general structure of the effective action in QFT in curved spacetime. This is in contradistinction to the vacuum structure of type-A models, where all included terms are expected. Overall, this feature might be indicative that the A-class of models are both theoretically and phenomenologically preferred to the B-ones. However, it is too early for a final verdict, and more observational work may be necessary to decide. In the meanwhile we have shown that the two types of models could be distinguished from the point of view of the measured redshift distribution of cluster-sized collapsed structures in the Universe. We have found that they can show significant deviations (of order $\pm 50 \%$ ) from the predicted redshift distribution in the concordance $\Lambda \mathrm{CDM}$ model. Our expectation is that when the upcoming and present X-ray and Sunyaev-Zeldovich surveys (such as eROSITA and SPT) will have collected enough statistics, it should be possible to decide about the best type of dynamical vacuum model from the phenomenological point of view.

In the course of our analysis we have also briefly pointed out the fact that generally the dynamical models under consideration in this paper, and especially when fitted using the 
$\mathrm{BAO}_{A}$ observable (which depends on low-redshift data on the acoustic $A(z)$-parameter), tend to provide a value of $\Omega_{m}^{0}$ significantly smaller than in the $\Lambda$ CDM model. This would seem to be consistent with the possible dynamical character of the dark energy recently claimed in the literature on the basis of model-independent DE diagnostics [33].

To summarize, the dynamical vacuum models of the cosmic evolution may offer an appealing and phenomenologically consistent perspective for describing dynamical dark energy without introducing extraneous dark energy fields. In that framework, dark energy is reinforced as being nothing more, but nothing less, than dynamical $\Lambda$. This could help to better understand the origin of the $\Lambda$-term and the vacuum energy density in the fundamental context of QFT in curved spacetime. Ultimately, it should shed light on the old cosmological constant problem, or at least provide a hint to elucidate the puzzling cosmic coincidence of the current matter and vacuum energy densities.

\section{Acknowledgments}

The work of AGV has been partially supported by an APIF predoctoral grant of the Universitat de Barcelona. JS has been supported in part by FPA2013-46570 (MICINN), Consolider grant CSD2007-00042 (CPAN) and by DIUE/CUR (Generalitat de Catalunya). SB acknowledges support by the Research Center for Astronomy of the Academy of Athens in the context of the program Tracing the Cosmic Acceleration.

\section{A. Understanding how the cluster number counts method works}

We have shown that type A and B models of the vacuum energy successfully fit all known cosmological data, including linear structure formation, in a way comparable to the $\Lambda$ CDM. However we would like to find a way to lift their alike performance and be able to distinguish them in a practical way. The number counts method is a good method to accomplish this aim. To understand semianalytically why the method works, it will suffice to consider the original Press-Schechter function defined in Sect.7.1. For convenience let us define the ratios

$$
\mathcal{T}(M) \equiv \frac{\int_{0}^{\infty} k^{n+2} T^{2}\left(\Omega_{m}^{(0)}, k\right) W^{2}(k R) d k}{\int_{0}^{\infty} k^{n+2} T^{2}\left(\Omega_{m}^{(0)}, k\right) W^{2}\left(k R_{8}\right) d k} \quad \text { and } \quad D_{N}(z) \equiv \frac{D(z)}{D(0)}
$$

In this way from (7.7) we have $\sigma^{2}(z)=\sigma_{8}^{2} D_{N}^{2}(z) \mathcal{T}(z)$, and we can rewrite (7.18) as follows:

$$
\mathcal{N}(z)=-\frac{4 \pi r^{2} \bar{\rho}}{H_{0} E(z)} \int_{M_{1}}^{M_{2}} \frac{1}{M}\left(\frac{1}{\sigma} \frac{d \sigma}{d M}\right) f_{P S c}(\sigma) d M=-4 \sqrt{2 \pi} \bar{\rho} \frac{c}{H_{0}}\left(\frac{\delta_{c}(z) r^{2}(z)}{E(z) \sigma_{8} D_{N}(z)}\right) I^{(\nu)}
$$


with $r(z)$ given in Eq.(7.17). In the last step we have used explicitly the original form of the Press-Schechter function $f_{P S c}(\sigma)$, and we have defined the integral

$$
I^{(\nu)} \equiv \int_{M_{1}}^{M_{2}} \frac{d M}{M} \frac{1}{\mathcal{T}} \frac{d \sqrt{\mathcal{T}}}{d M} e^{-\frac{\delta_{c}^{2}}{2 \sigma_{8}^{2} D_{N}^{2} \mathcal{T}}}
$$

Using the generalized forms (7.12) or (7.14) does not alter the explanation why the method works, and for this reason we restrict ourselves to the canonical one.

The variations with respect to the $\Lambda \mathrm{CDM}$ model should come from the variations in the terms in the big parenthesis on the r.h.s. of Eq. (A.2), as well as from the integral (A.3). The other ingredients of $\mathcal{N}(z)$ should not depend on the model details in a significant way. Let us assume that there is only one parameter in the dynamical vacuum model, say $\nu$. Expanding around $\nu=0$, i.e. around the $\Lambda$ CDM case, we can get the departure terms:

$$
\left(\frac{r^{2}(z)}{E(z)}\right)^{(\nu)}=\left(\frac{r^{2}(z)}{E(z)}\right)^{(0)}+\delta a_{1} ; \quad\left(\sigma_{8} D_{N}(z)\right)^{(\nu)}=\left(\sigma_{8} D_{N}(z)\right)^{(0)}+\delta a_{2} ; \quad \delta_{c}^{(\nu)}=\delta_{c}^{(0)}+\delta a_{3} .
$$

Notice that all the $\delta a_{i}$ in the previous expression are proportional to $\nu$, and therefore very small compared to the leading terms. Let us warn the reader that it would be inappropriate to expand the exponential in the integrand of (A.2) in the same way, as the linear approximation would be insufficient for the typical values of $\nu$ found in our analysis. The number counts formula (A.2) therefore yields

$$
\begin{aligned}
\mathcal{N}(z)= & -4 \sqrt{2 \pi} \bar{\rho} \frac{c}{H_{0}}\left(\frac{\delta_{c}(z) r^{2}(z)}{E(z) \sigma_{8} D_{N}(z)}\right)^{(0)} \\
& \times\left[1+\delta a_{1}\left(\frac{E(z)}{r^{2}(z)}\right)^{(0)}-\frac{\delta a_{2}}{\left(\sigma_{8} D_{N}(z)\right)^{(0)}}+\frac{\delta a_{3}}{\delta_{c}^{(0)}}+\mathcal{O}\left(\nu^{2}\right)\right] I^{(\nu)} .
\end{aligned}
$$

In this way we can compute the variation in the number of clusters (at a given redshift) with respect to the $\Lambda \mathrm{CDM}$, i.e. $\delta \mathcal{N}=\mathcal{N}(\nu)-\mathcal{N}(\nu=0)$. The corresponding relative variation can be cast as

$$
\frac{\delta \mathcal{N}}{\mathcal{N}}=\underbrace{\frac{I^{(\nu)}-I^{(0)}}{I^{(0)}}}_{T 0}+\underbrace{\delta a_{1}\left(\frac{E}{r^{2}}\right)^{(0)} \frac{I^{(\nu)}}{I^{(0)}}}_{T 1}-\underbrace{\frac{\delta a_{2}}{\left(\sigma_{8} D_{N}\right)^{(0)}} \frac{I^{(\nu)}}{I^{(0)}}}_{T 2}+\underbrace{\frac{\delta a_{3}}{\delta_{c}^{(0)} \frac{I^{(\nu)}}{I^{(0)}}}}_{T 3}, .
$$

The numerical evaluation of the various terms of this expression is displayed in Table 5 . It clearly shows that the dominant term is $T 0$ in (A.6). The terms $T 1-T 3$ are all of them proportional to $\delta a_{i}$ and hence to $\nu$. Since $\nu=\mathcal{O}\left(10^{-3}\right)$ all the terms proportional to it are of the same order of magnitude. The T0-term is not, and it becomes the leading one. Here is where the main contribution comes from, which is typically two orders of magnitude larger than $\nu$ and hence it can reach the order $10 \%$ rather than 1 per mil. This feature is at the root of the main difference of this method with respect to the linear 


\begin{tabular}{|c|c|c|c|c|c|c|c|c|}
\hline$\nu$ & $\sigma_{8}$ & $D_{N}(z=2)$ & $\delta_{c}(z=2)$ & $T 0$ & $T 1$ & $T 2$ & $T 3$ & $\frac{\delta \mathcal{N}}{\mathcal{N}}$ \\
\hline-0.0017 & 0.829 & 0.4315 & 1.695 & 0.096 & -0.0034 & -0.015 & 0.0065 & 0.084 \\
\hline 0.0017 & 0.794 & 0.4362 & 1.675 & -0.139 & 0.0027 & 0.016 & -0.0051 & -0.125 \\
\hline-0.004 & 0.854 & 0.4282 & 1.710 & 0.276 & -0.0092 & -0.047 & 0.0189 & 0.239 \\
\hline 0.004 & 0.770 & 0.4394 & 1.660 & -0.278 & 0.0052 & 0.030 & -0.0107 & -0.254 \\
\hline
\end{tabular}

Table 5: Numerical evaluation of $\delta \mathcal{N} / \mathcal{N}$, i.e. the relative variation in the number of counts as compared to the $\Lambda \mathrm{CDM}$, see Eq. (A.6). We consider different values of the $\nu$ parameter at fixed $z=2$ and provide also the breakdown of the result in the individual contributions $T 0-T 3$. To illustrate the method we have used the consistent set of inputs: $\Omega_{b}(z=0)=0.022242 h^{-2}$, $\Omega_{m}(z=0)=0.284, \delta_{c}^{(0)}=1.675, \sigma_{8}^{(0)}=0.811$ and $D_{N}^{(0)}(z=2)=0.4351$.

perturbations analysis. In the latter the deviations of the dynamical vacuum models with respect to the linear growth rate of the $\Lambda \mathrm{CDM}$ are proportional to $\nu$ and therefore cannot be distinguished. Here, instead, the relative differences become magnified thanks to the nonperturbative effects associated to (A.3). In addition, we note from Table 5 that there are significant differences for different values of $\nu$ within the same order of magnitude, which are also sensitive to sign changes of the parameter. In the present case the sign of $\delta \mathcal{N}$ is opposite to the sign of $\nu$, but this is because the value of $\Omega_{m}^{0}$ for the dynamical vacuum model that we have analyzed is the same as in the $\Lambda$ CDM, but in general there is no such sign correlation. What is important is that using the number count method we expect visible effects that would remain almost invisible in the linear approach owing to the small values of the model parameters. This is corroborated in the numerical analysis presented in Sect.7.2.

\section{B. Computing the collapse density threshold $\delta_{c}$ for the dynamical vacuum models}

In this appendix we present the necessary formulas to compute the linearly extrapolated density threshold above which structures collapse, i.e, $\delta_{c}$, for the type-A and type-B dynamical vacuum models under study. We follow the standard methods available in the literature - see e.g. [93] and [94], and references therein. Details of the procedure were also amply provided in Ref. [20] where it was applied to other dynamical vacuum models. We will therefore not repeat these details, but just the initial setup and the final results for the non-linear perturbation equations corresponding to the models under consideration. The linearized part of these equations reduces, of course, to the perturbations equations that we have derived previously in Section 5. Recall that in order to derive the nonlinear equations it is convenient to start from the Newtonian formalism for the cosmological fluid, and use the continuity, Euler and Poisson equations in the matter dominated epoch:

$$
\frac{\partial \rho_{m}}{\partial t}+\nabla_{\mathbf{r}} \cdot\left(\rho_{m} \mathbf{v}\right)=0
$$



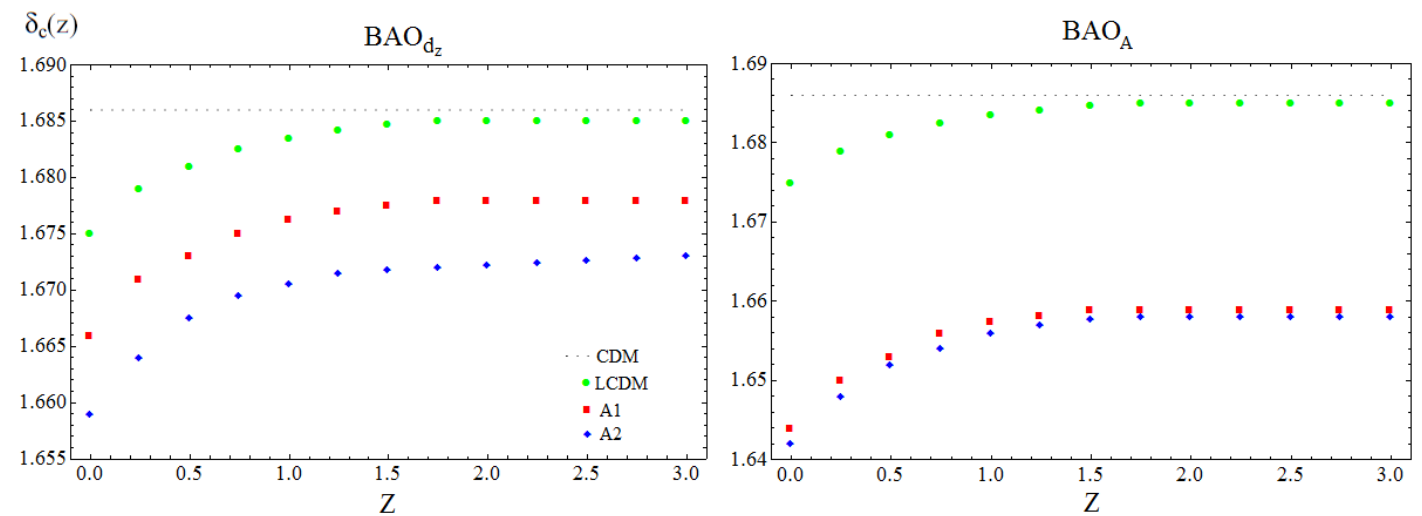

Figure 15: Computation of the collapse density threshold function $\delta_{c}(z)$ using the best fit values to $\mathrm{SNIa}+\mathrm{CMB}+\mathrm{BAO}_{d z}$ data (left plot) and to $\mathrm{SNIa}+\mathrm{CMB}+\mathrm{BAO}_{A}$ data (right plot). In both plots we include the constant $\mathrm{CDM}$ value $\delta_{c}=\frac{3}{20}(12 \pi)^{2 / 3} \simeq 1.686$ (horizontal dotted line) as well as the $\Lambda$ CDM curve (solid points, in green). The $\delta_{c}(z)$ curves for the vacuum models A1 and A2 are represented with squares (in red) and with diamonds (in blue), respectively. The corresponding values at $z=0$ define $\delta_{c}$ for each model, and are indicated in the last column of Table 3.

$$
\begin{gathered}
\frac{\partial \mathbf{v}}{\partial t}+\left(\mathbf{v} \cdot \nabla_{\mathbf{r}}\right) \mathbf{v}+\nabla_{\mathbf{r}} \Phi=0 \\
\nabla^{2} \Phi=4 \pi G_{N} \sum_{i} \rho_{i}\left(1+3 \omega_{i}\right),
\end{gathered}
$$

where $\mathbf{v}$ is the total velocity of the co-moving observer in three-space, $\Phi$ is the Newtonian gravitational potential, $\mathbf{r}$ is the physical coordinate, and $\omega_{i}=p_{i} / \rho_{i}$ is the EoS parameter for each component. Introducing comoving coordinates $\mathbf{x}=\mathbf{r} / a$ the perturbations are defined in the following way:

$$
\begin{aligned}
\rho_{i}(\mathbf{x}, t) & =\bar{\rho}_{i}(t)+\delta \rho_{i}(\mathbf{x}, t)=\bar{\rho}_{i}(t)\left(1+\delta_{i}(\mathbf{x}, t)\right), \\
\Phi(\mathbf{x}, t) & =\Phi_{0}(\mathbf{x}, t)+\phi(\mathbf{x}, t), \\
\mathbf{v}(\mathbf{x}, t) & =a(t)[H(t) \mathbf{x}+\mathbf{u}(\mathbf{x}, t)], .
\end{aligned}
$$

Here $\mathbf{u}(\mathbf{x}, t)$ is the comoving peculiar velocity. Next we have to insert inserts Eqs. (B.4)(B.6) into Eqs. (B.1)-(B.3) and use the definition of the gradient with respect to co-moving coordinates. Notice that $\nabla \delta_{m}=0$, which holds for the spherical collapse of a top-hat distribution. Following the same systematics as described in [20] we arrive at the nonlinear perturbations equations for the models under consideration.

\section{B.1 Type-A models}

In this case the corresponding fully non-linear evolution equation reads as follows:

$$
a^{2} H^{2} \delta_{m}^{\prime \prime}+a H \delta_{m}^{\prime}\left[3 H+Q-\frac{\rho_{m}}{2 H}\right]+\delta_{m}\left[2 H Q+a H Q^{\prime}-\frac{\rho_{m}}{2}\left(1+\delta_{m}\right)\right]-
$$




$$
-\left[\frac{4 a^{2} H^{2} \delta_{m}^{\prime 2}+5 a H Q \delta_{m} \delta_{m}^{\prime}+Q^{2} \delta_{m}^{2}}{3\left(1+\delta_{m}\right)}\right]=0
$$

where the primes continue denoting derivatives with respect to the scale factor and

$$
Q(a)=3 H(a)(1-\xi)
$$

The formulas for the non-relativistic matter energy density $\rho_{m}$ and the Hubble function $H$ can be found in Sect. 4.1. The numerical solution of the above nonlinear equation is used to compute $\delta_{c}(z)$ for models A1 and A2 in Fig. 15, see Sect. B.3 for details.

\section{B.2 Type-B models}

For this type of models the nonlinear equation for the perturbations can be obtained with some extra effort since on this occasion the calculations cannot be performed analytically in terms of the scale factor. We write the final result using the variable $y$, which has been defined in 5.15. We find:

$$
\begin{gathered}
\frac{9}{16} H_{0}^{2} \mathcal{F}^{2}(1-y)^{2} \delta_{m}^{\prime \prime}+\frac{3}{4} H_{0} \mathcal{F} \delta_{m}^{\prime}\left(1-y^{2}\right)\left[2 H+Q-\frac{3}{2} y H_{0} \mathcal{F}\right]+ \\
+\left[2 H Q+\frac{3}{4} H_{0} \mathcal{F}\left(1-y^{2}\right) Q^{\prime}-\frac{\rho_{m}}{2}\left(1+\delta_{m}\right)\right] \delta_{m}-\frac{Q^{2} \delta_{m}^{2}}{3\left(1+\delta_{m}\right)}- \\
-\left[\frac{4\left(\frac{3}{4} H_{0} \mathcal{F}\left(1-y^{2}\right) \delta_{m}^{\prime}\right)^{2}+\frac{15}{4} Q H_{0} \mathcal{F}\left(1-y^{2}\right) \delta_{m} \delta_{m}^{\prime}}{3\left(1+\delta_{m}\right)}\right]=0,
\end{gathered}
$$

where the primes indicate on this occasion derivatives with respect to $y$, defined in Eq. (5.15) - the notation should not be confusing with the previous use of primes since we make explicit the argument. The expressions for $Q(y), \rho_{m}(y)$ and $H(y)$ for the type-B models can be found in Sect.5.3. The numerical solution of the above nonlinear equation is used to compute $\delta_{c}(z)$ for models B1 and B2 in Fig. 16, cf. Sect. B.3.

The corresponding nonlinear equation for type-C1 models is a particular case of Eq. (B.9) and is obtained as indicated in Sect.4.3. In particular, for $\epsilon=\Omega_{\Lambda}^{0}$ and $\nu=0$ (hence $\left.\mathcal{F}=\Omega_{\Lambda}^{0}\right)$ we obtain the corresponding equation for the pure linear model $\rho_{\Lambda} \propto H$, which we have ruled out. We shall not consider the computation of the number counts for these models here.

\section{B.3 Numerical procedure to determine $\delta_{c}$}

Next we follow the prescriptions of [93], which was also described in detail (and applied to previous vacuum models) in [20]. We compute $\delta_{c}\left(z_{f}\right)$ by numerically integrating the above nonlinear equations between $z_{i}$ and $z_{f}$ (where the initial redshift $z_{i}$ is sufficiently large, for instance $\left.10^{6}\right)$. The aim is to find the initial value $\delta_{m}\left(z_{i}\right)$ for which the collapse takes place at $z=z_{f}$, i.e. such that $\delta_{m}\left(z_{f}\right)$ is very large, say $10^{5}$ or $10^{9}$ (the result does not change significantly). Second, we use the previously determined value of $\delta_{m}\left(z_{i}\right)$ together 

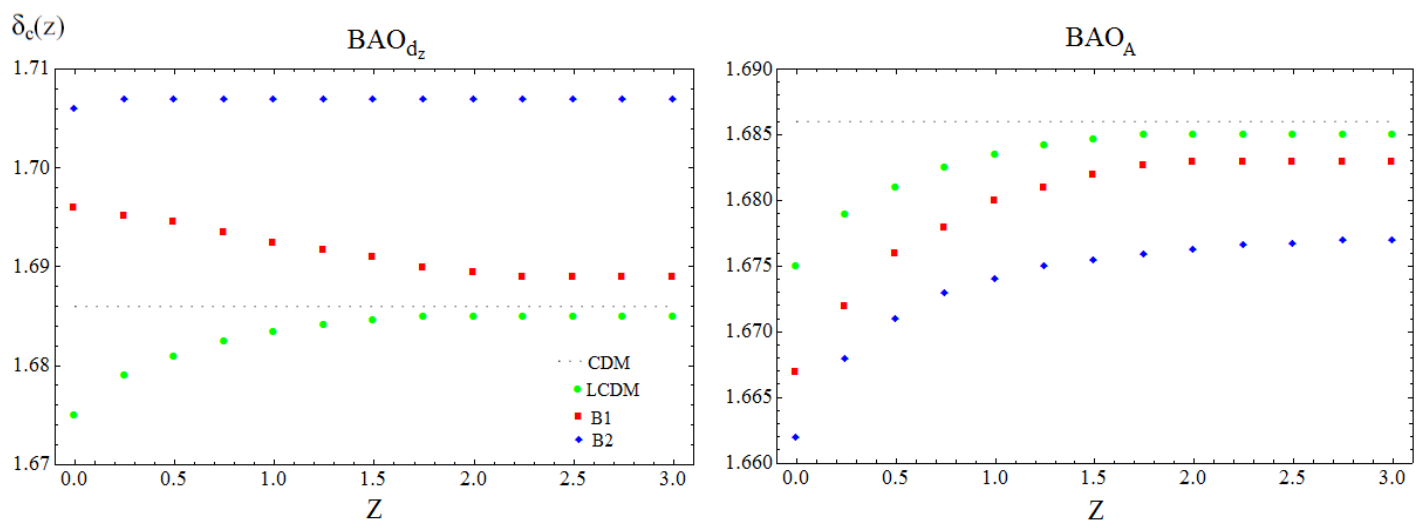

Figure 16: Computation of the collapse density threshold function $\delta_{c}(z)$ for the B1 and B2 vacuum models. The rest of the notation is as in Fig. 15. The values of $\delta_{c}(z)$ at $z=0$ are indicated in the last column of Table 4.

with a small value of $\delta_{m}^{\prime}\left(z_{i}\right)$. In fact, we know it is zero for a sphere, so we may take $\delta_{m}^{\prime}\left(z_{i}\right) \sim 10^{-6}-10^{-4}[93]$. These are then used as the initial conditions for solving the corresponding linear perturbations equations. The value of $\delta_{m}\left(z_{f}\right)$ obtained in the second step of this procedure defines $\delta_{c}\left(z_{f}\right)$, and the value of this quantity at $z_{f}=0$ defines $\delta_{c} \equiv \delta_{c}\left(z_{f}=0\right)$. Notice that the linear equations are simply obtained from (B.7) and (B.9) upon neglecting all terms of $\mathcal{O}\left(\delta_{m}^{2}\right)$ and $\mathcal{O}\left(\delta_{m}^{\prime 2}\right)$. The equations obtained in this way are, of course, the ones already presented in Sect. 5 for both types of models A and B. The values of $\delta_{c}$ obtained by this method for each model are displayed in the last column of Tables 3 and 4 (cf. Sect. 7.2). The numerical solutions $\delta_{c}(z)$ for each model are displayed in Figs. 15 and 16 .

\section{References}

[1] Planck Collaboration (P.A.R. Ade et al.), Planck 2013 results. XVI. Cosmological parameters, Astron. Astrophys. (2014) [e-Print: arXiv:1303.5076].

[2] S. Weinberg, Rev. Mod. Phys. 61 (1989) 1; V. Sahni, A. Starobinsky, Int. J. of Mod. Phys. A9 (2000) 373; T. Padmanabhan, Phys. Rep. 380 (2003) 235.

[3] J. Solà, Cosmological constant and vacuum energy: old and new ideas, J. Phys. Conf. Ser. (2013) 453012015 [e-Print: arXiv:1306.1527]; Vacuum energy and cosmological evolution, AIP Conf.Proc. 1606 (2014) 19 [e-Print: arXiv:1402.7049], and references therein.

[4] P.J. Peebles, B. Ratra, Rev. Mod. Phys. 75 (2003) 559.

[5] S. Nojiri and S.D. Odintsov, eConf C0602061 (2006) 06, Int. J. Geom. Meth. Mod. Phys. 4 (2007) 115 [e-Print: arXiv:hep-th/0601213]; T.P. Sotiriou, V. Faraoni, Rev. Mod. Phys. 82 (2010) 451 [e-Print: arXiv:0805.1726]; R. Woodard, Lect. Notes Phys. 720 (2007) 403 [e-Print: arXiv:astro-ph/0601672]; S. Capozziello, and M. De Laurentis, Phys. Rept. 509 (2011) 167 [e-Print: arXiv:1108.6266]. 
[6] F. Bauer, J. Solà, H. Štefančić, JCAP 1012 (2010) 029 [e-Print: arXiv:1006.3944]; Phys. Lett. B688 (2010) 269 [e-Print: arXiv:0912.0677]; Mod. Phys. Lett. A26 (2011) 2559 [e-Print: arXiv:1105.1030]; Phys. Lett. B678 (2009) 427 [e-Print: arXiv:0902.2215]; S. Basilakos, F. Bauer, and J. Solà, JCAP 1201 (2012) 050 [e-Print: arXiv:1109.4739].

[7] N.D. Birrell and P.C.W. Davies, Quantum Fields in Curved Space (Cambridge U. Press, 1982).

[8] L.E. Parker and D.J. Toms, Quantum Field Theory in Curved Spacetime: quantized fields and gravity (Cambridge U. Press, 2009).

[9] I. L. Shapiro, and J. Solà, JHEP 0202 (2002) 006 [e-Print: arXiv:hep-th/0012227]; Phys. Lett. B475 (2000) 236 [e-Print: hep-ph/9910462]; Phys.Lett. B530 (2002) 10 [e-Print: hep-ph/0104182]; Nucl. Phys. Proc. Suppl. 127 (2004) 71 [e-Print: hep-ph/0305279]; PoS AHEP2003 (2003) 013 [e-Print: astro-ph/0401015]; J. of Phys. A40 (2007) 6583 [e-Print: arXiv: gr-qc/0611055], and references therein;

[10] A. Babić, B. Guberina, R. Horvat, H. Štefančić, Phys. Rev. D65 (2002) 085002 [e-Print: hep-ph/0111207]; Phys. Rev. D71 (2005) 124041 [e-Print: astro-ph/0407572].

[11] J. Solà, H. Štefančić, Phys. Lett. B624 (2005) 147 [e-Print: astro-ph/0505133]; Mod. Phys. Lett. A21 (2006) 479 [e-Print: astro-ph/0507110].

[12] J. Solà, J. of Phys. A41 (2008) 164066 [e-Print: arXiv:0710.4151].

[13] I. L. Shapiro, and J. Solà, Phys. Lett. B682 (2009) 105 [e-Print: arXiv:0910.4925]; confer also the extended version arXiv:0808.0315, and references therein.

[14] J. Solà, Cosmologies with a time dependent vacuum, J. Phys. Conf. Ser. 283 (2011) 012033 [e-Print: arXiv:1102.1815]; Fortsch. Phys. 59 (2011) 1108; and references therein.

[15] O. Bertolami, Nuovo Cimento, 93B, 36, (1986); M. Ozer M. and O. Taha, Nucl. Phys., B287, 776, (1987); O. K. Freese K., et al., Nucl. Phys., 287, 797, (1987); J. C. Carvalho, J. A. S. Lima and I. Waga, Phys. Rev. D46, 2404, (1992). See e.g. the reviews: J. M. Overduin and F. I. Cooperstock, Phys. Rev. D58 (1998) 043506; and R.G. Vishwakarma, Class. Quant. Grav. 18 (2001) 1159, and references therein.

[16] P. Wang, X.-H. Meng, Class. Quant. Grav. 22 (2005) 283 [e-Print: astro-ph/0408495].

[17] J. S. Alcaniz, J.A.S. Lima, Phys.Rev. D72 (2005) 063516 [e-Print: astro-ph/0507372].

[18] C. España-Bonet et al., JCAP 0402 (2004) 006; Phys.Lett. B574 (2003) 149.

[19] S. Basilakos, M. Plionis and J. Solà, Phys. Rev. D80, (2009) 3511 [e-Print: arXiv:0907.4555].

[20] J. Grande, J. Solà, S. Basilakos, and M. Plionis, JCAP 08 (2011) 007 [e-Print: arXiv:1103.4632].

[21] S. Basilakos, D. Polarski, and J. Solà, Phys. Rev. D86 (2012) 043010 [e-Print: arXiv:1204.4806].

[22] S. Basilakos, and J. Solà, Mon. Not. Roy. Astron. Soc. 437 (2014) 3331 [e-Print: arXiv:1307.4748]. 
[23] S. Basilakos, and J. Solà, Phys. Rev. D90 (2014) 023008 [e-Print: arXiv:1402.6594].

[24] J. C. Fabris, I. L. Shapiro, J. Solà, JCAP 0702 (2007) 016 [e-Print: gr-qc/0609017]; J. Grande, J. Solà, J.C. Fabris, I.L.Shapiro, Class. Quant. Grav. 27 (2010) 105004 [e-Print: arXiv:1001.0259]; A. M. Velasquez-Toribio, Int. J. of Mod. Phys. D21 (2012) 1250026 [e-Print: arXiv:0907.3518].

[25] J. Grande, A. Pelinson and J. Solà, Phys.Rev. D79 (2009) 043006 [e-Print: arXiv:0809.3462].

[26] J. Grande, A. Pelinson and J. Solà, Proc. of the 7th International Heidelberg Conference on Dark Matter in Astro and Particle Physics (DARK 2009), e-Print: arXiv:0904.3293.

[27] J. Grande, J. Solà and H. Štefančić, JCAP 08 (2006) 011 [e-Print: gr-qc/0604057]; Phys. Lett. B645 (2007) [e-Print: gr-qc/0609083].

[28] J. Grande, R. Opher, A. Pelinson, and J. Solà, JCAP 0712 (2007) 007 [e-Print: arXiv:0709.2130].

[29] M. Maggiore, Phys. Rev. D83 (2011) 063514 [e-Print: arXiv:1004.1782]; M.S. Sloth, Int.J.Mod.Phys. D19 (2010) 2259 [e-Print: arXiv:1005.3241]; L. Hollenstein, M. Jaccard, M. Maggiore, E. Mitsou, Phys. Rev. D85 (2012) [e-Print: arXiv:1111.5575].

[30] N. Bilić, Phys. Rev. D83 (2011) 105003 [e-Print: arXiv:1104.1349]; Phys. Lett. B707 (2012) 221 [e-Print: arXiv:1110.2393]; N. Bilić, S. Domazet, B. Guberina, Phys.Lett. B707 (2012) 221 [e-Print: arXiv:1110.2393].

[31] J. A. S. Lima, S. Basilakos, and J. Solà, Mon. Not. Roy. Astron. Soc. 431 (2013) 923 [e-Print: arXiv:1209.2802]; E. L. D. Perico, J.A.S. Lima, S. Basilakos, and J. Solà, Phys. Rev. D88 (2013) 063531 [e-Print: arXiv:1306.0591]; S. Basilakos, J. A. S. Lima, and J. Solà, Int. J. Mod. Phys. D (2013) [e-Print: arXiv:1307.6251].

[32] S. Basilakos, J. A. S. Lima, J. Solà, Int. J. of Mod. Phys. D23 (2014) 12, 1442011 [e-Print: arXiv:1406.2201].

[33] V. Sahni, A. Shafieloo, A. A. Starobinsky, Model independent evidence for dark energy evolution from Baryon Acoustic Oscillations, Astrophys. J. 793 (2014) L40 [e-Print: arXiv:1406.2209].

[34] J.K. Webb, M.T. Murphy, V.V. Flambaum, V.A. Dzuba, J. D. Barrow, C.W. Churchill, J.X. Prochaska, A.M. Wolfe, Phys. Rev. Lett. 87 (2001) 091301 [e-Print: astro-ph/0012539].

[35] J-P. Uzan, Living Rev. Rel. 14 (2011) 2 [e-Print: arXiv:1009.5514]; T. Chiba, Prog. Theor. Phys. 126 (2011) 993 [e-Print: arXiv:1111.0092].

[36] H. Fritzsch, J. Solà, Class. Quant. Grav. 29 (2012) 215002 [e-Print: arXiv:1202.5097]; H. Fritzsch, and J. Solà, Advances in High Energy Physics 2014 (2014) 361587 [e-Print: arXiv:1402.4106].

[37] J. Solà, Int. J. of Mod. Phys. A29 (2014) 1444016 [e-Print: arXiv:1408.4427].

[38] P. J. E. Peebles, Principles of Physical Cosmology (Princeton Univ. Press, Princeton New Jersey, 1993).

[39] A. Pouri, S. Basilakos, M. Plionis, JCAP 1408 (2014) 042 [e-Print: arXiv:1402.0964]. 
[40] A. Merloni et al., eROSITA Science Book: Mapping the Structure of the Energetic Universe, e-Print: arXiv:1209.3114.

[41] J.E. Carlstrom et al., Publ. Astron. Soc. Pac. 123 (2011) 568 [e-Print: arXiv:0907.4445].

[42] L.E. Bleem et al., Galaxy Clusters Discovered via the Sunyaev-Zel'dovich Effect in the 2500-square-degree SPT-SZ survey, e-Print: arXiv:1409.0850.

[43] S. Basilakos, M. Plionis and J. Solà, Phys. Rev. D82 (2010) 083512 [e-Print: arXiv:1005.5592].

[44] I. L. Shapiro, Class.Quant.Grav. 25 (2008) 103001 [e-Print: arXiv:0801.0216]

[45] J. A. S. Lima, M. Trodden Phys. Rev. D 53 (1996) 4280.

[46] G.L. Murphy, Phys. Rev. D8 (1973) 4231; J. D. Barrow, Nucl. Phys. B310 (1988) 743; J. A. S. Lima, R. Portugal, and I. Waga, Phys. Rev.D37 (1988) 2755; W. Zimdahl, Phys. Rev. D53 (1996) 5483; I. Brevik and S. D. Odintsov, Phys. Rev. D65 067302 (2002); B. Li and J. D. Barrow, Phys. Rev. D79 (2009) 103521.

[47] J. Ren, X-H. Meng, Phys. Lett. B633 (2006) 1 [e-Print: astro-ph/0511163]; Int. J. Mod. Phys. D16 (2007) 1341 [e-Print: astro-ph/0605010].

[48] N. Komatsu, S. Kimura, Phys.Rev. D88 (2013) 083534 [e-Print: arXiv:1307.5949].

[49] R. Schutzhold, Phys. Rev. Lett. 89 (2002) 081302; F.R. Klinkhamer, G.E. Volovik, Phys.Rev. D79 (2009) 063527; N. Ohta, Phys. Lett. B 695 (2011) 41.

[50] A. R. Zhitnitsky [e-Print: arXiv:1310.2258]; E. Thomas, A. R. Zhitnitsky, Phys.Rev. D86 (2012) 065029 [e-Print: arXiv:1203.6073]; Phys.Rev. D87 (2013) 085027 [e-Print: arXiv:1208.2030]; E.C. Thomas, F. R. Urban, A. R. Zhitnitsky, JHEP 0908 (2009) 043 [e-Print: arXiv:0904.3779].

[51] S. Carneiro, M.A. Dantas, C. Pigozzo, J.S. Alcaniz, Phys.Rev. D77 (2008) 083504 [e-Print: arXiv:0711.2686]; J.S. Alcaniz, H.A. Borges, S. Carneiro, J.C. Fabris, C. Pigozzo, W. Zimdahl, Phys.Lett. B716 (2012) 165 [e-Print: arXiv:1201.5919].

[52] N. ChandrachaniDevi, H.A. Borges, S. Carneiro, J.S. Alcaniz. e-Print: arXiv:1407.1821.

[53] E.P. Verlinde, JHEP 1104 (2011) 029 [e-Print: arXiv:1001.0785].

[54] D. A. Easson, P. H. Frampton, G.F. Smoot, Phys. Lett. B696 (2011) 273 [e-Print: arXiv:1002.4278].

[55] D. A. Easson, P. H. Frampton, G.F. Smoot, Int.J.Mod.Phys. A27 (2012) 1250066 [e-Print: arXiv:1003.1528].

[56] R. C. Arcuri and I. Waga., Phys. Rev. D. 50 (1994) 2928.

[57] N. Komatsu, S. Kimura, Phys. Rev. D89 (2014) 123501 [e-Print: arXiv:1402.3755]. Phys. Rev. D87 (2013) 043531 [e-Print: arXiv:1208.2482].

[58] N. Komatsu, S. Kimura, Entropic cosmology in a dissipative universe, e-Print: arXiv:1408.4836.

[59] L.N. Granda, A. Oliveros, Phys.Lett. B669 (2008) 275 [e-Print: arXiv:0810.3149]. 
[60] T. S. Koivisto, D. F. Mota, M. Zumalacarregui, JCAP 1102 (2011) 027 [e-Print: arXiv:1011.2226]; M. Visser, JHEP 1110 (2011) 140 [e-Print: arXiv:1108.5240].

[61] R. Casadio, A. Gruppuso, Phys. Rev. D84 (2011) 023503 [e-Print: arXiv:1005.0790]; Y-F. Cai, J. Liu, H. Li, Phys. Lett. B690 (2010) 213 [e-Print: arXiv:1003.4526]; H. Wei Phys. Lett. B692 (2010) 167 [e-Print: arXiv:1005.1445]; Y.S. Myung, Astrophys. Space Sci. 335 (2011) 553 [e-Print: arXiv:1005.2240]; Y. Fu Cai, E. N. Saridakis, Phys. Lett. B697 (2011) 280 [e-Print: arXiv:1011.1245].

[62] S. Dodelson, Modern Cosmology (Academic Press, 2003).

[63] I.S. Gradshteyn, I.M. Ryzhik, Table of integrals, series, and products (Academic Press, 1980).

[64] N. Suzuki, D. Rubin, C. Lidman, G. Aldering, R. Amanullah, K. Barbary, L. F. Barrientos and J. Botyanszki et al., Astrophys. J 746 (2012) 85.

[65] J. R. Bond, G. Efstathiou and M. Tegmark, Mon. Not. Roy. Astron. Soc. 291 (1997) L33 [e-Print: arXiv:astro-ph/9702100].

[66] S. Nesseris and L. Perivolaropoulos, JCAP 0701 (2007) 018 [e-Print: arXiv:astro-ph/0610092].

[67] D. L. Shaefer and D. Huterer, Phys.Rev. D89 (2014) 063510 [e-Print:arXiv:1312.1688].

[68] W. Hu, N. Sugiyama, Astrophys. J. 444 (1995) 489 [e-Print: astro-ph/9510117].

[69] D. J. Eisenstein et al. (SDSS Collab.), Astrophys. J., 633 (2005) 560 [e-Print: astro-ph/0501171]; N. Padmanabhan, et al. (SDSS Collab.), Mon. Not. Roy. Astron. Soc., 378 (2007) 852 [e-Print: astro-ph/0605302].

[70] W. J. Percival, Mon. Not. Roy. Astron. Soc., 401 (2010) 2148 [e-Print: arXiv:0907.1660].

[71] C. Blake et al., Mon. Not. Roy. Astron. Soc., 418 (2011) 1707 [e-Print: arXiv:1108.2635].

[72] D. J. Eisenstein, and W. Hu, Astrophys. J. 496 (1998) 605 [e-Print: astro-ph/9709112].

[73] H. Akaike, IEEE Transactions of Automatic Control, 19 (1974) 716; N. Sugiura, Communications in Statistics A, Theory and Methods, 7 (1978) 13.

[74] M. Hicken et al., Astroplys. J., 700 (2009) 1097 [e-Print: arXiv:0901.4804].

[75] M. Chevallier, D. Polarski, Int. J. Mod. Phys. D10 (2001) 10 [e-Print: gr-qc/0009008]; E. V. Linder, Phys. Rev. Lett., 90 (2003) 091301 [e-Print: astro-ph/0208512].

[76] J. Valiviita, R. Maartens, E. Majerotto, Mon. Not. Roy. Astron. Soc. 402 (2010) [e-Print: arXiv:0907.4987].

[77] V. Sahni, A. Shafieloo, A.A. Starobinsky, Phys. Rev. D78 (2008) 103502 [e-Print: arXiv:0807.3548].

[78] Y. Hu, Miao Li, Z. Zhang, e-Print: arXiv:1406.7695.

[79] T. Delubac. et al., Baryon Acoustic Oscillations in the Lya forest of BOSS DR11 quasars, e-Print: arXiv:1404.1801. 
[80] S. Basilakos, S. Nesseris and L. Perivolaropoulos, Phys. Rev. D., 87 (2013) 123529 [e-Print: arXiv:1302.6051].

[81] Y-S. Song and W.J. Percival, JCAP 0910 (2009) 004 [e-Print: arXiv:0807.0810].

[82] L-M. Wang, P. J. Steinhardt, Astrophys. J. 508 (1998) 483 [e-Print: astro-ph/9804015].

[83] S. Basilakos, A. Gómez and J. Solà (work in progress).

[84] S. Basilakos, M. Plionis, J.A.S. Lima, Phys.Rev. D82 (2010) 083517 [e-Print: arXiv:1006.3418].

[85] L. Campanelli, G.L. Fogli, T. Kahniashvili, A. Marrone, B. Ratra, Eur. Phys. J. C72 (2012) 2218 [e-Print: arXiv:1110.2310].

[86] N.ChandrachaniDevi, T.R. Choudhury, A. A. Sen, Mon.Not.Roy.Astron.Soc. 432 (2013) 1513 [e-Print: arXiv:1112.0728]; N.ChandrachaniDevi, J.E. Gonzalez, J.S. Alcaniz, JCAP 1406 (2014) 055 [e-Print: arXiv:1402.2590].

[87] W.H. Press and P. Schechter, Astrophys. J. 187 (1974) 425.

[88] V. Eke, S. Cole \&, C. S. Frenk, Mon. Not. Roy. Astron. Soc., 282 (1996) 263 [e-Print: astro-ph/9601088].

[89] J. M. Bardeen, J. R. Bond, N. Kaiser, and, A. S. Szalay, Astrophys. J., 304 (1986) 15.

[90] A.R. Liddle, D. H. Lyth, Cosmological Inflation and Large-Scale Structure (Cambridge Univ. Press, 2000).

[91] D. Reed, R. Bower, C. Frenk, A. Jenkins, and T. Theuns, MNRAS 374 (2007) 2 [e-Print: astro-ph/0607150].

[92] R.K. Sheth, G. Tormen, Mon. Not. Roy. Astron. Soc. 308 (1999) 119 [e-Print: astro-ph/9901122].

[93] F. Pace, J.-C Waizmann and M. Bartelmann, Mon. Not. Roy. Astron. Soc. 406 (2010) 1865 [e-Print: arXiv:1005.0233].

[94] L.R. Abramo, R.C. Batista, L. Liberato, R. Rosenfeld, JCAP 0711, 012 (2007) [e-Print: arXiv:0707.2882]; Phys.Rev. D79 (2009) 023516 [e-Print: arXiv:0806.3461]. 